\title{
TOWARDS A QUANTIZATION OF THE DOUBLE VIA THE ENHANCED SYMPLECTIC "CATEGORY"
}

\author{
PETER CROOKS AND JONATHAN WEITSMAN
}

\begin{abstract}
This paper considers the enhanced symplectic "category" for purposes of quantizing quasi-Hamiltonian $G$-spaces, where $G$ is a compact simple Lie group. Our starting point is the well-acknowledged analogy between the cotangent bundle $T^{*} G$ in Hamiltonian geometry and the internally fused double $D(G)=G \times G$ in quasi-Hamiltonian geometry. Guillemin and Sternberg consider the former, studing half-densities and phase functions on its so-called character Lagrangians $\Lambda_{\mathcal{O}} \subseteq T^{*} G$. Our quasi-Hamiltonian counterpart replaces these character Lagrangians with the universal centralizers $\Lambda_{\mathcal{C}} \longrightarrow \mathcal{C}$ of regular, $\frac{1}{k}$-integral conjugacy classes $\mathcal{C} \subseteq G$. We show each universal centralizer to be a "quasi-Hamiltonian Lagrangian" in $D(G)$, and to come equipped with a half-density and phase function.

At the same time, we consider a Dehn twist-induced automorphism $R: D(G) \longrightarrow D(G)$ that lacks a natural Hamiltonian analogue. Each quasi-Hamiltonian Lagrangian $R\left(\Lambda_{\mathcal{C}}\right)$ is shown to have a clean intersection with every $\Lambda_{\mathcal{C}^{\prime}}$, and to come equipped with a half-density and phase function of its own. This leads us to consider the possibility of a well-behaved, quasi-Hamiltonian notion of the BKS pairing between $R\left(\Lambda_{\mathcal{C}}\right)$ and $\Lambda_{\mathcal{C}^{\prime}}$. We construct such a pairing and study its properties. This is facilitated by the nice geometric fearures of $R\left(\Lambda_{\mathcal{C}}\right) \cap \Lambda_{\mathcal{C}^{\prime}}$ and a reformulation of the classical BKS pairing. Our work is perhaps the first step towards a level- $k$ quantization of $D(G)$ via the enhanced symplectic "category".
\end{abstract}

\section{Contents}

1. Introduction

1.1. Context

1.2. Statement of results

1.3. Organization

Acknowledgements

2. Quantization and symplectic "categories"

2.1. The Weinstein symplectic "category"

2.2. Examples and basic properties

2.3. The enhanced symplectic "category"

2.4. BKS pairings

3. Lie-theoretic constructions

3.1. The basics

3.2. Adjoint orbits

3.3. Haar measures on maximal tori

3.4. The phase function on $\Lambda_{\mathcal{O}}$

3.5. The half-density on $\Lambda_{\mathcal{O}}$

4.1. Quasi-Hamiltonian $G$-spaces 16

2020 Mathematics Subject Classification. 53D20 (primary); 57S15 (secondary).

Key words and phrases. geometric quantization, quasi-Hamiltonian G-space, symplectic "category". 
4.2. The internally fused double

4.3. The gauge-theoretic realization of $D(G)$

4.4. The symplectic submanifold $T \times T$

4.5. A special instance of quasi-Hamiltonian reduction

4.6. Universal centralizers of conjugacy classes

5. BKS pairings on the internally fused double

5.1. The geometry of $R\left(\Lambda_{\mathcal{C}}\right) \cap \Lambda_{\mathcal{C}^{\prime}}$

5.2. Half-densities on $R\left(\Lambda_{\mathcal{C}}\right)$ and $\Lambda_{\mathcal{C}^{\prime}}$

5.3. Phase functions on $R\left(\Lambda_{\mathcal{C}}\right)$ and $\Lambda_{\mathcal{C}^{\prime}}$

5.4. Some technical lemmas

5.5. The main results

A1. Some preliminaries

A2. Exact sequences

A3. The BKS density

A4. Some technical results

A5. A reformulation of the BKS density

A6. A result on half-densities and $G$-invariance

References

\section{INTRODUCTION}

1.1. Context. It is a premise of the geometric quantization programme that some symmetries of a symplectic manifold $M$ should induce symmetries of the quantization $Q(M)$. This can be captured via the formalism of symplectic "categories" [16], through which geometric quantizations are related to the intersection theory of Lagrangian submanifolds. Guillemin and Sternberg [6] study a variant of this construction, replacing the symplectic "category" with a decorated version called the enhanced symplectic "category". Lagrangian relations are thereby replaced with arrows of the form

$$
M_{1} \stackrel{(\Lambda, \rho)}{\Longrightarrow} M_{2},
$$

where $\Lambda$ is a Lagrangian relation and $\rho$ is a half-density on $\Lambda$. This is exemplified by a class of Lagrangian submanifolds in $T^{*} G$, where $G$ is a compact 1-connected simple Lie group having Lie algebra $\mathfrak{g}$. The Lagrangian submanifolds in question are the so-called character Lagrangians $\Lambda_{\mathcal{O}} \subseteq T^{*} G$, where $\mathcal{O} \subseteq \mathfrak{g}^{*}$ ranges over the regular integral coadjoint orbits of $G$. Guillemin and Sternberg [6] endow each $\Lambda_{\mathcal{O}}$ with a distsinguished half-density $\rho_{\mathcal{O}}$ and phase function $\psi_{\mathcal{O}}: \Lambda_{\mathcal{O}} \longrightarrow$ $S^{1}$.

Several papers [2, 7-12, 14, 15] address the quantization of quasi-Hamiltonian $G$-spaces [1. A recurring theme is that the approaches to geometric quantization in Hamiltonian geometry have counterparts in the quasi-Hamiltonian setting. One may therefore expect that the enhanced symplectic "category" will lend itself to the quantization of quasi-Hamiltonian $G$-spaces. One example is the internally fused double $D(G)=G \times G$ [1, a quasi-Hamiltonian $G$-space and the quasiHamiltonian analogue the Hamiltonian $G$-space $T^{*} G$. The natural analogues of the character Lagrangians $\Lambda_{\mathcal{O}} \subseteq T^{*} G$ are then the universal centralizers $\Lambda_{\mathcal{C}} \subseteq D(G)$, where $\mathcal{C} \subseteq G$ ranges over the regular, $\frac{1}{k}$-integral conjugacy classes of $G$ and $k$ is a fixed positive integer. The role of $\Lambda_{\mathcal{O}}$ in a 
quantization of $T^{*} G$ via the enhanced symplectic "category" might therefore suggest a role for $\Lambda_{\mathcal{C}}$ in quantizing $D(G)$.

1.2. Statement of results. This paper investigates the possibility of quantizing $D(G)$ via universal centralizers $\Lambda_{\mathcal{C}} \subseteq D(G)$ and the enhanced symplectic "category". We do not construct a geometric quantization itself; our emphasis is instead on the interesting geometric features of $D(G)$ and $\Lambda_{\mathcal{C}}$ that would be relevant to a quantization of $D(G)$ via the enhanced symplectic "category". One such feature is a Dehn twist automorphism $R: D(G) \longrightarrow D(G)$ that lacks a natural Hamiltonian analogue. Our main technical tool is a generalized Blattner-Kostant-Sternberg (BKS) pairing [4] that applies in both symplectic and quasi-Hamiltonian geometry. The following is a more detailed summary of our results.

1.2.1. A generalization of the classical BKS pairing. We generalize the classical BKS pairing to suit our particular purposes. Our generalization applies to pairs $(M, \omega)$ with $\omega$ an arbitrary two-form on $M$. We let $M^{\circ} \subseteq M$ denote the open submanifold of points at which $\omega$ is non-degenerate, noting that $M^{\circ}$ is even-dimensional. A submanifold $\Lambda \subseteq M$ is then declared to be Lagrangian if $\Lambda \subseteq M^{\circ}$, $\operatorname{dim}(\Lambda)=\frac{1}{2} \operatorname{dim}\left(M^{\circ}\right)$, and $\Lambda$ is isotropic with respect to $\omega$. This leads to the following observation.

Observation 1.2.1. Let $(M, \omega)$ consist of a manifold $M$ and an arbitrary two-form $\omega \in \Omega^{2}(M)$. Suppose that $\Lambda_{1}, \Lambda_{2} \subseteq M$ are Lagrangian submanifolds in the sense defined above, and that they come equipped with respective half-densities $\rho_{1}, \rho_{2}$ and smooth maps $\psi_{1}: \Lambda_{1} \longrightarrow \mathbb{C}, \psi_{2}: \Lambda_{2} \longrightarrow \mathbb{C}$. Let us also assume that $\Lambda_{1}$ and $\Lambda_{2}$ have a clean intersection in $M$, and that $\Lambda_{1} \cap \Lambda_{2}$ is compact. The usual definition of the BKS pairing

$$
\operatorname{BKS}\left(\left(\Lambda_{1}, \rho_{1}, \psi_{1}\right),\left(\Lambda_{2}, \rho_{2}, \psi_{2}\right)\right) \in \mathbb{C}
$$

for symplectic $(M, \omega)$ generalizes to our setting.

Our interest in Observation 1.2.1 stems from its applicability to quasi-Hamiltonian $G$-spaces $(M, \omega)$.

1.2.2. Lagrangians in the internally fused double. Let $G$ be a compact, connected, simply-connected, simple Lie group with Lie algebra $\mathfrak{g}$, exponential map exp $: \mathfrak{g} \longrightarrow G$, and adjoint representation Ad $: G \longrightarrow \mathrm{GL}(\mathfrak{g})$. Fix a maximal torus $T \subseteq G$ having Lie algebra $\mathfrak{t} \subseteq \mathfrak{g}$ and Weyl group $W:=N_{G}(T) / T$. Denote by $\langle\cdot, \cdot\rangle: \mathfrak{g} \otimes_{\mathbb{R}} \mathfrak{g} \longrightarrow \mathbb{R}$ the unique $G$-invariant inner product for which each long root has length $\sqrt{2}$, noting that $\langle\cdot, \cdot\rangle$ identifies $\mathfrak{t}$ with $\mathfrak{t}^{*}$.

Use the left trivialization and $\langle\cdot, \cdot\rangle$ to identify $T^{*} G$ with $G \times \mathfrak{g}$. Each regular adjoint orbit $\mathcal{O} \subseteq \mathfrak{g}$ then determines a Lagrangian submanifold $\Lambda_{\mathcal{O}} \subseteq T^{*} G$, i.e.

$$
\Lambda_{\mathcal{O}}:=\left\{(g, \xi) \in G \times \mathfrak{g}: \xi \in \mathcal{O} \text { and } \operatorname{Ad}_{g}(\xi)=\xi\right\} \subseteq G \times \mathfrak{g}=T^{*} G .
$$

One may instead consider a regular conjugacy class $\mathcal{C} \subseteq G$ and its universal centralizer $\Lambda_{\mathcal{C}} \subseteq$ $G \times G=D(G)$, defined by

$$
\Lambda_{\mathcal{C}}:=\left\{(g, h) \in D(G): h \in \mathcal{C} \text { and } g h g^{-1}=h\right\} .
$$

We show this universal centralizer to a quasi-Hamiltonian counterpart of $\Lambda_{\mathcal{O}}$ in the following sense.

Theorem 1.2.2. Let $\mathcal{C} \subseteq G$ be a regular conjugacy class. The universal centralizer $\Lambda_{\mathcal{C}}$ is a Lagrangian submanifold of the internally fused double $D(G)$, i.e. $\Lambda_{\mathcal{C}} \subseteq D(G)^{\circ}$, $\operatorname{dim}\left(\Lambda_{\mathcal{C}}\right)=n$, and $\Lambda_{\mathcal{C}}$ is isotropic with respect to the quasi-Hamiltonian 2-form on $D(G)$. 
1.2.3. BKS pairings on the internally fused double. Now choose a collection of simple roots and let $\mathfrak{A} \subseteq \mathfrak{t}$ be the associated fundamental Weyl alcove. Let $\beta(\mathcal{C})$ denote the unique element of $\mathfrak{A}$ satisfying $\exp (\beta(\mathcal{C})) \in \mathcal{C}$, where $\mathcal{C} \subseteq G$ is any conjugacy class. Let us also fix a positive integer $k$ and assume that $\mathcal{C}$ is a regular, $\frac{1}{k}$-integral conjugacy class, i.e. that $k \beta(\mathcal{C})$ is a regular, integral weight in $\mathfrak{t} \cong \mathfrak{t}^{*}$.

Write $\mathcal{O} \subseteq \mathfrak{g}$ for the adjoint orbit of $k \beta(\mathcal{C})$, and noting that

$$
\Lambda_{\mathcal{O}} \longrightarrow \Lambda_{\mathcal{C}}, \quad(g, \xi) \mapsto\left(g, \exp \left(\frac{1}{k} \xi\right)\right), \quad(g, \xi) \in \Lambda_{\mathcal{O}}
$$

is a diffeomorphism. On the other hand, Guillemin and Sternberg equip $\Lambda_{\mathcal{O}}$ with a canonical halfdensity $\rho_{\mathcal{O}}$ and phase function $\psi_{\mathcal{O}}: \Lambda_{\mathcal{O}} \longrightarrow S^{1}$. By means of the above-mentioned diffeomorphism, these determine a half-density $\rho_{\mathcal{C}}$ and function $\psi_{\mathcal{C}}: \Lambda_{\mathcal{C}} \longrightarrow S^{1}$ on $\Lambda_{\mathcal{C}}$. We will ultimately compute the BKS pairing of $\left(\Lambda_{\mathcal{C}}, \rho_{\mathcal{C}}, \psi_{\mathcal{C}}\right)$ with another triple, constructed via the following ideas.

Certain gauge-theoretic considerations naturally endow $D(G)$ with an action of the mapping class group of the once-punctured torus, an action that respects the quasi-Hamiltonian $G$-space structure on $D(G)$. This mapping class group contains two Dehn twist automorphisms, one of which acts on $D(G)$ through the diffeomorphism

$$
R: D(G) \longrightarrow D(G), \quad(g, h) \mapsto\left(g, h g^{-1}\right), \quad(g, h) \in D(G) .
$$

It follows that $R\left(\Lambda_{\mathcal{C}}\right)$ is a Lagrangian submanifold of $D(G)$. Note also that the restricted diffeomorphism

$$
\left.R\right|_{\Lambda_{\mathcal{C}}}: \Lambda_{\mathcal{C}} \stackrel{\cong}{\longrightarrow} R\left(\Lambda_{\mathcal{C}}\right)
$$

identifies $\rho_{\mathcal{C}}$ (resp. $\psi_{\mathcal{C}}$ ) with a half-density $\nu_{\mathcal{C}}$ (resp. phase function $\vartheta_{\mathcal{C}}$ ) on $R\left(\Lambda_{\mathcal{C}}\right.$ ). We thereby obtain the triple $\left(R\left(\Lambda_{\mathcal{C}}\right), \nu_{\mathcal{C}}, \vartheta_{\mathcal{C}}\right)$ alluded to in the previous paragraph. This leads to the following BKS pairing computation.

Theorem 1.2.3. Fix a positive integer $k$ and let $\mathcal{C}, \mathcal{C}^{\prime} \subseteq G$ be regular, $\frac{1}{k}$-integral conjugacy classes.

(i) The Lagrangian submanifolds $R\left(\Lambda_{\mathcal{C}}\right)$ and $\Lambda_{\mathcal{C}^{\prime}}$ have a clean intersection in $D(G)$.

(ii) The BKS pairing of $\left(R\left(\Lambda_{\mathcal{C}}\right), \nu_{\mathcal{C}}, \vartheta_{\mathcal{C}}\right)$ and $\left(\Lambda_{\mathcal{C}^{\prime}}, \rho_{\mathcal{C}^{\prime}}, \psi_{\mathcal{C}^{\prime}}\right)$ is given by

$\operatorname{BKS}\left(\left(R\left(\Lambda_{\mathcal{C}}\right), \nu_{\mathcal{C}}, \vartheta_{\mathcal{C}}\right),\left(\Lambda_{\mathcal{C}^{\prime}}, \rho_{\mathcal{C}^{\prime}}, \psi_{\mathcal{C}^{\prime}}\right)\right)=k^{n-r} C(G, T) \cdot\left(\prod_{\alpha \in \Phi_{+}} \alpha(\beta(\mathcal{C})) \alpha\left(\beta\left(\mathcal{C}^{\prime}\right)\right)\right)^{\frac{1}{2}}\left(\sum_{w \in W} e^{2 \pi i\left\|w \beta-\beta^{\prime}\right\|^{2}}\right)$,

where $n=\operatorname{dim}(G), r=\operatorname{rank}(G), \Phi_{+} \subseteq \mathfrak{t}^{*}$ is the set of positive roots, $C(G, T) \in \mathbb{R}$ is a constant depending only on the root system of $(G, T)$, and $\left\|w \beta-\beta^{\prime}\right\|$ is the length of $w \beta-\beta^{\prime}$ with respect to $\langle\cdot, \cdot\rangle$.

1.3. Organization. Section 2 uses the framework of symplectic "categories" to motivate the investigations undertaken in this paper. Section 3 then develops the requisite Lie-theoretic machinery. This leads to Section 4, which studies the quasi-Hamiltonian geometry of $\Lambda_{\mathcal{C}}$ and includes a proof of Theorem 1.2.2. In Section 5, we study the geometric features of $R\left(\Lambda_{\mathcal{C}}\right) \cap \Lambda_{\mathcal{C}^{\prime}}$ and prove Theorem 1.2.3. Our final section is an appendix that develops several linear-algebraic and differentialgeometric properties of BKS pairings. A list of recurring notation appears at the end of this paper.

Acknowledgements. P.C. was supported by an NSERC postdoctoral fellowship [PDF-516638], while J.W. was supported by a Simons collaboration grant [\#579801]. 


\section{Quantization and symplectic "CATEgories"}

The following section emphasizes the connection between geometric quantization and the intersection theory of Lagrangian submanifolds. This connection is our impetus for studying the quasi-Hamiltonian Lagrangians $R\left(\Lambda_{\mathcal{C}}\right), \Lambda_{\mathcal{C}^{\prime}} \subseteq D(G)$ in relation to one another. One can realize this connection via Weinstein's symplectic "category" [16] and an enrichment thereof [6]; such "categories" are related to the intersection theory of Lagrangian submanifolds, and to geometric quantization itself. We review the first of these relations, deferring to [3] and [6] for the latter.

2.1. The Weinstein symplectic "category". Let $(M, \omega)$ be any symplectic manifold. In what follows, we adopt the shorthand notation $M$ for $(M, \omega)$ and $\bar{M}$ for the symplectic manifold $(M,-\omega)$.

Suppose that $M_{1}$ and $M_{2}$ are symplectic manifolds. Recall that a Lagrangian relation from $M_{1}$ to $M_{2}$ is a Lagrangian submanifold $\Lambda \subseteq \overline{M_{1}} \times M_{2}$, where $\overline{M_{1}} \times M_{2}$ is equipped with the usual product symplectic form. In this case, one writes

$$
M_{1} \stackrel{\Lambda}{\Longrightarrow} M_{2}
$$

Let $M_{1}, M_{2}$, and $M_{3}$ be symplectic manifolds. Given Lagrangian relations

$$
M_{1} \stackrel{\Lambda_{12}}{\Longrightarrow} M_{2} \text { and } M_{2} \stackrel{\Lambda_{23}}{\Longrightarrow} M_{3},
$$

one defines

$$
\Lambda_{23} \circ \Lambda_{12}:=\left\{\left(m_{1}, m_{3}\right) \in M_{1} \times M_{3}: \exists m_{2} \in M_{2} \text { with }\left(m_{1}, m_{2}\right) \in \Lambda_{12} \text { and }\left(m_{2}, m_{3}\right) \in \Lambda_{23}\right\} .
$$

Note that $\Lambda_{23} \circ \Lambda_{12}$ need not be a submanifold of $M_{1} \times M_{3}$; one typically addresses this by requiring $\Lambda_{23}$ and $\Lambda_{12}$ to be cleanly composable, an intersection-theoretic condition discussed at length in [6, Section 4.2]. This condition forces $\Lambda_{23} \circ \Lambda_{12}$ to be a submanifold and a Lagrangian relation, called the composition of $\Lambda_{23}$ and $\Lambda_{12}$ and denoted

$$
M_{1} \stackrel{\Lambda_{12}}{\Longrightarrow} M_{2} \stackrel{\Lambda_{23}}{\Longrightarrow} M_{3}
$$

It is tempting to imagine a category with symplectic manifolds as objects, Lagrangian relations as morphisms, and morphism composition as defined above. This is precluded by the existence of Lagrangian relations whose composition fails to be a submanifold. One instead refers to symplectic manifolds and Lagrangian relations as forming the Weinstein symplectic "category", and views morphism composition as defined only for cleanly composable relations.

\subsection{Examples and basic properties.}

(1) If $M$ is a symplectic manifold, then Lagrangian submanifolds of $\Lambda \subseteq M$ are in bijective correspondence with Lagrangian relations of the form

$$
\mathrm{pt} \stackrel{\Lambda}{\Longrightarrow} M \text {. }
$$

(2) If $M$ is a symplectic manifold, then the diagonal $\Delta_{M} \subseteq M \times M$ is a Lagrangian submanifold of $\bar{M} \times M$. By the previous example, it defines a Lagrangian relation

$$
\mathrm{pt} \stackrel{\Delta_{M}}{\Longrightarrow} \bar{M} \times M \text {. }
$$

(3) Suppose that $G$ is a compact Lie group with Lie algebra $\mathfrak{g}$. Let $M$ be a symplectic manifold endowed with a free Hamiltonian $G$-action and associated moment map $\mu: M \longrightarrow \mathfrak{g}^{*}$. Consider the symplectic quotient $\mu^{-1}(0) / G$ and canonical map

$$
\pi: \mu^{-1}(0) \longrightarrow \mu^{-1}(0) / G \text {. }
$$


The graph of $\pi$ is then a Lagrangian relation

$$
M \stackrel{\Lambda}{\Longrightarrow} \mu^{-1}(0) / G
$$

i.e.

$$
\Lambda:=\left\{(m, \pi(m)): m \in \mu^{-1}(0)\right\} \subseteq M \times \mu_{D(G)}^{-1}(0) / G .
$$

One often calls $\Lambda$ the reduction morphism.

(4) Retain the hypotheses and notation of the previous example, except for the freeness condition on the $G$-action. Use the left trivialization to identify $T^{*} G$ with $G \times \mathfrak{g}^{*}$, and consider

$$
\Lambda_{\mu}:=\{(m, g \cdot m, g, \mu(m)): m \in M, g \in G\} \subseteq M \times M \times G \times \mathfrak{g}^{*}=M \times M \times T^{*} G .
$$

This is a Lagrangian submanifold of $M \times \bar{M} \times T^{*} G$, and it thereby defines a Lagrangian relation

$$
\bar{M} \times M \stackrel{\Lambda_{\mu}}{\Longrightarrow} T^{*} G \text {. }
$$

One sometimes calls $\Lambda_{\mu}$ the moment Lagrangian.

(5) Suppose that $G$ is a compact Lie group with Lie algebra $\mathfrak{g}$, adjoint representation Ad : $G \longrightarrow$ $\mathrm{GL}(\mathfrak{g})$, and fixed $G$-invariant inner product $\langle\cdot, \cdot\rangle: \mathfrak{g} \otimes \mathbb{R} \mathfrak{g} \longrightarrow \mathbb{R}$. This inner product induces an isomorphism between the adjoint and coadjoint representations of $G$, through which we may freely identify $\mathfrak{g}$ and $\mathfrak{g}^{*}$. Each adjoint orbit $\mathcal{O} \subseteq \mathfrak{g}$ thereby corresponds to a coadjoint orbit, and the latter comes equipped with its canonical Kirillov-Kostant-Souriau symplectic form. It follows that $\mathcal{O}$ is a symplectic manifold, and one sees that the $G$-action on $\mathcal{O}$ is Hamiltonian with moment map the inclusion $\mu: \mathcal{O} \longrightarrow \mathfrak{g}$. One can also verify that the Lagrangian relations

$$
\mathrm{pt} \stackrel{\Delta_{\mathcal{O}}}{\Longrightarrow} \overline{\mathcal{O}} \times \mathcal{O} \text { and } \overline{\mathcal{O}} \times \mathcal{O} \stackrel{\Lambda_{\mu}}{\Longrightarrow} T^{*} G
$$

are cleanly composable, so that we have a Lagrangian relation

$$
\mathrm{pt} \stackrel{\Lambda_{\mu \circ \Delta} \mathcal{O}}{\Longrightarrow} T^{*} G \text {. }
$$

This amounts to having a Lagrangian submanifold $\Lambda_{\mathcal{O}} \subseteq T^{*} G$. If one uses the left trivialization and $\langle\cdot, \cdot\rangle$ to identify $T^{*} G$ with $G \times \mathfrak{g}$, then

$$
\Lambda_{\mathcal{O}}=\left\{(g, \xi) \in G \times \mathfrak{g}: \xi \in \mathcal{O} \text { and } \operatorname{Ad}_{g}(\xi)=\xi\right\} .
$$

This submanifold is often called the orbit Lagrangian.

2.3. The enhanced symplectic "category". Guillemin and Sternberg [6] discuss an enrichment of the Weinstein symplectic "category". This involves replacing Lagrangian relations

$$
M_{1} \stackrel{\Lambda}{\Longrightarrow} M_{2}
$$

with arrows of the form

$$
M_{1} \stackrel{(\Lambda, \rho)}{\Longrightarrow} M_{2}
$$

where $\Lambda \subseteq \overline{M_{1}} \times M_{2}$ is a Lagrangian relation from $M_{1}$ to $M_{2}$ and $\rho$ is a half-density on $\Lambda$. Now suppose that $M_{1}, M_{2}$, and $M_{3}$ are symplectic manifolds with arrows

$$
M_{1} \stackrel{\left(\Lambda_{\left.12, \rho_{12}\right)}\right.}{\Longrightarrow} M_{2} \text { and } M_{2} \stackrel{\left(\Lambda_{23}, \rho_{23}\right)}{\Longrightarrow} M_{3} .
$$

Assuming that $\Lambda_{23}$ and $\Lambda_{12}$ are cleanly composable as Lagrangian relations, Guillemin and Sternberg construct a half-density $\rho_{23} \circ \rho_{12}$ on the Lagrangian submanifold $\Lambda_{23} \circ \Lambda_{12} \subseteq \overline{M_{1}} \times M_{3}$. The composition of the arrows (11) is then defined to be $(\Lambda, \rho)=\left(\Lambda_{23} \circ \Lambda_{12}, \rho_{23} \circ \rho_{12}\right)$, i.e. the new arrow

$$
M_{1} \stackrel{(\Lambda, \rho)}{\Longrightarrow} M_{3} .
$$


The enhanced symplectic "category" is defined to have symplectic manifolds as objects, arrows of the form

$$
M_{1} \stackrel{(\Lambda, \rho)}{\Longrightarrow} M_{2}
$$

as morphisms, and morphism composition as defined above. This fails to form a category, as one composes morphisms only if the underlying Lagrangian relations are cleanly composable.

2.4. BKS pairings. The enhanced symplectic "category" is compatible with the so-called BlattnerKostant-Sternberg $(\mathrm{BKS})$ pairing [4] in geometric quantization. To elaborate on this, we let $(V, \omega)$ be a $2 n$-dimensional symplectic vector space over $\mathbb{R}$. Recall that a density of order $\alpha \in(0, \infty)$ on an $m$-dimensional subspace $W \subseteq V$ is a map $\rho: W^{m} \longrightarrow \mathbb{C}$ satisfying

$$
\rho\left(A\left(v_{1}\right), \ldots, A\left(v_{m}\right)\right)=|\operatorname{det}(A)|^{\alpha} \rho\left(v_{1}, \ldots, v_{m}\right)
$$

for all $A \in \operatorname{End}(W)$ and $\left(v_{1}, \ldots, v_{m}\right) \in W^{m}$. We use the term density (resp. half-density) for a density of order one (resp. $\frac{1}{2}$ ), and write $|W|^{\alpha}$ for the one-dimensional complex vector space of densities of order $\alpha$ on $W$. Let us also write $|W|$ for $|W|^{\alpha}$ in the case $\alpha=1$. One crucial ingredient of BKS theory is the existence of a canonical isomorphism

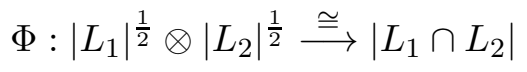

for any pair of Lagrangian subspaces $L_{1}, L_{2} \subseteq V$. A more detailed discussion appears in the appendix.

Now let $(M, \omega)$ be a symplectic manifold with cleanly intersecting Lagrangian submanifolds $\Lambda_{1}, \Lambda_{2} \subseteq M$. Assume that $\Lambda_{1} \cap \Lambda_{2}$ is compact, and that $\Lambda_{1}$ and $\Lambda_{2}$ come with respective halfdensities $\rho_{1}$ and $\rho_{2}$ and smooth functions $\psi_{1}: \Lambda_{1} \longrightarrow \mathbb{C}$ and $\psi_{2}: \Lambda_{2} \longrightarrow \mathbb{C}$. The half-densities $\rho_{1}$ and $\rho_{2}$ are sections of appropriate half-density bundles. One may therefore apply (2) on the level of tangent spaces to obtain a density $D\left(\rho_{1}, \rho_{2}\right)$ on $\Lambda_{1} \cap \Lambda_{2}$. The BKS pairing of $\left(\Lambda_{1}, \rho_{1}, \psi_{1}\right)$ and $\left(\Lambda_{2}, \rho_{2}, \psi_{2}\right)$ is then defined by

$$
\operatorname{BKS}\left(\left(\Lambda_{1}, \rho_{1}, \psi_{1}\right),\left(\Lambda_{2}, \rho_{2}, \psi_{2}\right)\right):=\int_{\Lambda_{1} \cap \Lambda_{2}} \psi_{1} \overline{\psi_{2}}
$$

where the integration is with respect to $D\left(\rho_{1}, \rho_{2}\right)$.

\section{LIE-THEORETIC CONSTRUCTIONS}

In what follows, we introduce and develop the Lie-theoretic underpinnings of our work.

3.1. The basics. Let $G$ be a compact, connected, simply-connected, $n$-dimensional, rank- $r$, simple Lie group with Lie algebra $\mathfrak{g}$, exponential map exp $: \mathfrak{g} \longrightarrow G$, and adjoint representation Ad $: G \longrightarrow$ $\mathrm{GL}(\mathfrak{g})$. Write $G_{g}$ for the $G$-centralizer of $g \in G$ and

$$
G_{\text {reg }}:=\left\{g \in G: \operatorname{dim}\left(G_{g}\right)=r\right\}
$$

for the open, dense, conjugation-invariant subset of regular elements in $G$. A conjugacy class $\mathcal{C} \subseteq G$ shall be called regular if $\mathcal{C} \subseteq G_{\text {reg, }}$, i.e. if $\mathcal{C}$ is the conjugacy class of a regular element in $G$.

We likewise write $G_{x} \subseteq G$ (resp. $\mathfrak{g}_{x} \subseteq \mathfrak{g}$ ) for the $G$-stabilizer (resp. $\mathfrak{g}$-centralizer) of $x \in \mathfrak{g}$ and

$$
\mathfrak{g}_{\text {reg }}:=\left\{x \in \mathfrak{g}: \operatorname{dim}\left(\mathfrak{g}_{x}\right)=r\right\}
$$

for the open, dense, $G$-invariant subset of regular elements in $\mathfrak{g}$. An adjoint orbit $\mathcal{O} \subseteq \mathfrak{g}$ shall be called regular if $\mathcal{O} \subseteq \mathfrak{g}_{\text {reg }}$, i.e. if $\mathcal{O}$ is the adjoint orbit of a regular element in $\mathfrak{g}$. 
Some of the preceding discussion fits into the following more general framework. Let $M$ be a $G$-manifold, i.e. a manifold equipped with a smooth left action of $G$. Each $m \in M$ then determines a $G$-stabilizer

and $G$-orbit

$$
G_{m}:=\{g \in G: g \cdot m=m\} \subseteq G
$$

$$
G m:=\{g \cdot m: g \in G\} \subseteq M .
$$

Let $T \subseteq G$ be a maximal torus with Lie algebra $\mathfrak{t} \subseteq \mathfrak{g}$, setting

$$
T_{\text {reg }}:=T \cap G_{\text {reg }} \quad \text { and } \quad \mathfrak{t}_{\text {reg }}:=\mathfrak{t} \cap \mathfrak{g}_{\text {reg }} \text {. }
$$

It follows that $t \in T$ (resp. $x \in \mathfrak{t}$ ) belongs to $T_{\text {reg }}\left(\right.$ resp. $\mathfrak{t}_{\text {reg }}$ ) if and only if $G_{t}=T$ (resp. $\mathfrak{g}_{x}=\mathfrak{t}$ ).

Let $X^{\bullet}(T)$ denote the weight lattice of all Lie group morphisms $T \longrightarrow S^{1}$. Each $\alpha \in X^{\bullet}(T)$ can be differentiated at $e \in T$ to yield an $\mathbb{R}$-linear map

$$
(d \alpha)_{e}: \mathfrak{t} \longrightarrow T_{1} S^{1}=i \mathbb{R} .
$$

The map

$$
X^{\bullet}(T) \longrightarrow \mathfrak{t}^{*}, \quad \alpha \mapsto \frac{1}{2 \pi i}(d \alpha)_{e}, \quad \alpha \in X^{\bullet}(T)
$$

is then an embedding of $\mathbb{Z}$-modules. Elements in its image are called integral weights.

Let us set $\mathfrak{g}_{\mathbb{C}}:=\mathfrak{g} \otimes_{\mathbb{C}} \mathbb{R}$ and write $\operatorname{Ad}^{\mathbb{C}}: G \longrightarrow \mathrm{GL}\left(\mathfrak{g}_{\mathbb{C}}\right)$ for the complexification of the adjoint representation. Recall that $\alpha \in X^{\bullet}(T) \backslash\{0\}$ is called a root if

$$
\mathfrak{g}_{\alpha}:=\left\{\xi \in \mathfrak{g}_{\mathbb{C}}: \operatorname{Ad}_{t}^{\mathbb{C}}(\xi)=\alpha(t) \xi \text { for all } t \in T\right\} \subseteq \mathfrak{g}_{\mathbb{C}}
$$

is one-dimensional. If one uses (3) to regard roots as being in $\mathfrak{t}^{*}$, then $\alpha \in \mathfrak{t}^{*} \backslash\{0\}$ is a root if and only if

$$
\mathfrak{g}_{\alpha}:=\left\{\xi \in \mathfrak{g}_{\mathbb{C}}:[\eta, \xi]=2 \pi i \alpha(\eta) \xi \text { for all } \eta \in \mathfrak{t}\right\} \subseteq \mathfrak{g}_{\mathbb{C}}
$$

is one-dimensional. This leads to the $T$-module decomposition

$$
\mathfrak{g}_{\mathbb{C}}=\mathfrak{t}_{\mathbb{C}} \oplus \bigoplus_{\alpha \in \Phi} \mathfrak{g}_{\alpha}
$$

where $\mathfrak{t}_{\mathbb{C}}:=\mathfrak{t} \otimes_{\mathbb{R}} \mathbb{C}$ and $\Phi$ is the set of all roots. Choose a decomposition $\Phi=\Phi_{+} \cup \Phi_{-}$, where $\Phi_{+}$ is a set of positive roots and $\Phi_{-}=-\Phi_{+}$is the corresponding set of negative roots. Let $\Delta \subseteq \Phi_{+}$ denote the resulting set of simple roots, and write $\alpha_{0} \in \Phi_{+}$for the highest root.

Let $\langle\cdot, \cdot\rangle: \mathfrak{g} \otimes_{\mathbb{R}} \mathfrak{g} \longrightarrow \mathbb{R}$ denote the normalized inner product on $\mathfrak{g}$. This is the unique $G$-invariant inner product satisfying a certain normalization condition, to be stated momentarily. Note that $\langle\cdot, \cdot\rangle$ induces an isomorphism

$$
\mathfrak{g} \stackrel{\cong}{\longrightarrow} \mathfrak{g}^{*}, \quad \xi \mapsto\langle\xi, \cdot\rangle, \quad \xi \in \mathfrak{g}
$$

between the adjoint representation and its dual. Our inner product also yields a vector space isomorphism

$$
\mathfrak{t} \stackrel{\cong}{\longrightarrow} \mathfrak{t}^{*}, \quad \xi \mapsto \xi^{\vee}:=\left.\langle\xi, \cdot\rangle\right|_{\mathfrak{t}}, \quad \xi \in \mathfrak{t}
$$

through which $\mathfrak{t}^{*}$ acquires an inner product. Abusing notation slightly, we also use $\langle\cdot, \cdot\rangle$ to denote this new inner product. Our normalization condition on $\langle\cdot, \cdot\rangle: \mathfrak{g} \otimes_{\mathbb{R}} \mathfrak{g} \longrightarrow \mathbb{R}$ is then given by

$$
\left\langle\alpha_{0}, \alpha_{0}\right\rangle=2
$$

Now consider the conjugation action of $G$ on itself, as well as its canonical lift to a Hamiltonian $G$-action on $T^{*} G$. If one uses $\langle\cdot, \cdot\rangle$ and the left trivialization to identify $T^{*} G$ with $G \times \mathfrak{g}$, then this latter action is given by

$$
g \cdot(h, x)=\left(g h g^{-1}, \operatorname{Ad}_{g}(x)\right)
$$


for all $g \in G$ and $(h, x) \in G \times \mathfrak{g}$. This is the only $G$-action on $T^{*} G$ that we will consider.

Recall that $\alpha \in \mathfrak{t}^{*}$ is called dominant if $\langle\alpha, \beta\rangle \geq 0$ for all $\beta \in \Delta$. We refer to $\xi \in \mathfrak{t}$ as being dominant if $\xi^{\vee}$ is so, i.e. $\xi \in \mathfrak{t}$ is dominant if and only if $\alpha(\xi) \geq 0$ for all $\alpha \in \Delta$. We likewise call an element $\xi \in \mathfrak{t}$ integral if $\xi^{\vee}$ is an integral weight in $\mathfrak{t}^{*}$. An adjoint orbit $\mathcal{O} \subseteq \mathfrak{g}$ shall be called integral if it contains an integral element of $t$.

Let $W:=N_{G}(T) / T$ be the Weyl group associated to $(G, T)$. Recall that $W$ acts linearly on $\mathfrak{t}$ through the adjoint representation of $G$. The induced $W$-action on $\mathfrak{t}^{*}$ preserves $X^{\bullet}(T)$ and $\Phi$, and we have a length function $\ell: W \longrightarrow \mathbb{Z}_{\geq 0}$ defined by

$$
\ell(w):=\left|\Phi_{+} \cap w^{-1} \Phi_{-}\right|, \quad w \in W .
$$

Consider the fundamental Weyl alcove $\mathfrak{A} \subseteq \mathfrak{t}$ defined by

$$
\mathfrak{A}:=\left\{\xi \in \mathfrak{t}: \alpha_{0}(\xi) \leq 1 \text { and } \alpha(\xi) \geq 0 \text { for all } \alpha \in \Delta\right\} .
$$

One has a bijection

$$
\mathfrak{A} \stackrel{\cong}{\longrightarrow} \text { conjugacy classes in } G\}, \quad \xi \mapsto \mathcal{C}_{\exp (\xi)}, \quad \xi \in \mathfrak{A},
$$

where $\mathcal{C}_{\exp (\xi)} \subseteq G$ denotes the conjugacy class of $\exp (\xi)$. Let

$$
\beta:\{\text { conjugacy classes in } G\} \stackrel{\cong}{\longrightarrow} \mathfrak{A}
$$

be the inverse bijection, i.e. $\beta(\mathcal{C})$ is the unique element of $\mathfrak{A}$ that exponentiates to an element of $\mathcal{C}$. Let us also suppose that $k$ is a positive integer. We then refer to a conjugacy class $\mathcal{C} \subseteq G$ as being regular (resp. $\frac{1}{k}$-integral) if $\beta(\mathcal{C})$ (resp. $k \beta(\mathcal{C})$ ) is a regular (resp. an integral) element of $\mathfrak{t}$. These notions features prominently in our work.

3.2. Adjoint orbits. Fix $\xi \in \mathfrak{g}$ and let $\mathcal{O} \subseteq \mathfrak{g}$ denote its adjoint orbit. Note that the isomorphism (4) identifies $\mathcal{O}$ with a coadjoint orbit in $\mathfrak{g}^{*}$. The Kirillov-Kostant-Souriau symplectic form on the latter orbit thereby corresponds to a symplectic form $\omega_{\mathcal{O}}$ on $\mathcal{O}$, which we now describe.

Let us write $V^{\perp}$ for the annihilator of a subspace $V \subseteq \mathfrak{g}$ with respect to $\langle\cdot, \cdot\rangle$. We may identify $T_{\xi} \mathcal{O}$ with $\mathfrak{g}_{\xi}^{\perp}$ in the usual way, i.e. via the isomorphism that sends each vector in $\mathfrak{g}_{\xi}^{\perp}$ to its fundamental vector field on $\mathcal{O}$ at $\xi$. Let $\omega_{\xi}$ denote the value of $\omega_{\mathcal{O}}$ at $\xi$, by which we mean

$$
\omega_{\xi}:=\left(\omega_{\mathcal{O}}\right)_{\xi} \in \wedge^{2}\left(\left(\mathfrak{g}_{\xi}^{\perp}\right)^{*}\right) \cong \wedge^{2}\left(\left(T_{\xi} \mathcal{O}\right)^{*}\right) .
$$

The form $\omega_{\mathcal{O}}$ is then characterized by being $G$-invariant and satisfying

$$
\omega_{\xi}\left(\eta_{1}, \eta_{2}\right)=\left\langle\xi,\left[\eta_{1}, \eta_{2}\right]\right\rangle
$$

for all $\eta_{1}, \eta_{2} \in \mathfrak{g}_{\xi}^{\perp} \cong T_{\xi} \mathcal{O}$.

Let $\Omega_{\mathcal{O}}$ denote the highest non-zero wedge power of $\omega_{\mathcal{O}}$ and set $\Omega_{\xi}:=\left(\Omega_{\mathcal{O}}\right)_{\xi}$. Note that

$$
\Omega_{\xi} \in \wedge^{n-r}\left(\left(\mathfrak{g}_{\xi}^{\perp}\right)^{*}\right) \cong \wedge^{n-r}\left(\left(T_{\xi} \mathcal{O}\right)^{*}\right)
$$

if $\xi \in \mathfrak{g}_{\mathrm{reg}}$.

Lemma 3.2.1. If $\xi \in \mathfrak{t}_{\text {reg }}$ and $w \in W$, then

$$
\Omega_{w \xi}=(-1)^{\ell(w)} \Omega_{\xi}
$$

as multilinear forms on $\mathfrak{t}^{\perp}$.

Proof. Choose root vectors $\left\{e_{\alpha} \in \mathfrak{g}_{\alpha}\right\}_{\alpha \in \Phi_{+}}$and $\left\{e_{-\alpha} \in \mathfrak{g}_{-\alpha}\right\}_{\alpha \in \Phi_{+}}$so that

$$
\left\{i\left(e_{\alpha}+e_{-\alpha}\right)\right\}_{\alpha \in \Phi_{+}} \cup\left\{e_{\alpha}-e_{-\alpha}\right\}_{\alpha \in \Phi_{+}}
$$


is an orthonormal basis of $\mathfrak{t}^{\perp}$. Let us record these basis vectors as

$$
x_{\alpha}:=i\left(e_{\alpha}+e_{-\alpha}\right) \quad \text { and } \quad y_{\alpha}:=e_{\alpha}-e_{-\alpha}
$$

for each $\alpha \in \Phi_{+}$. In the interest of what lies ahead, we note the following straightforward identity:

$$
\left[\xi, x_{\alpha}\right]=-2 \pi \alpha(\xi) y_{\alpha}
$$

for all $\alpha \in \Phi_{+}$.

Now note that

$$
\begin{aligned}
& \omega_{\xi}\left(x_{\alpha}, x_{\beta}\right)=\left\langle\xi,\left[x_{\alpha}, x_{\beta}\right]\right\rangle \\
& =\left\langle\left[\xi, x_{\alpha}\right], x_{\beta}\right\rangle \\
& =-2 \pi \alpha(\xi)\left\langle y_{\alpha}, x_{\beta}\right\rangle \quad \text { [by (하)] } \\
& =0 \quad \text { [by orthogonality] }
\end{aligned}
$$

for all $\alpha, \beta \in \Phi_{+}$. A similar argument establishes that $\omega_{\xi}\left(y_{\alpha}, y_{\beta}\right)=0$ for all $\alpha, \beta \in \Phi_{+}$. On the other hand,

$$
\begin{aligned}
\omega_{\xi}\left(x_{\alpha}, y_{\beta}\right) & =\left\langle\xi,\left[x_{\alpha}, y_{\beta}\right]\right\rangle \\
& =\left\langle\left[\xi, x_{\alpha}\right], y_{\beta}\right\rangle \\
& =-2 \pi \alpha(\xi)\left\langle y_{\alpha}, y_{\beta}\right\rangle \quad[\text { by (6) }]
\end{aligned}
$$

for all $\alpha, \beta \in \Phi_{+}$. We conclude that $\omega_{\xi}\left(x_{\alpha}, y_{\beta}\right)$ is zero if $\alpha \neq \beta$, and that it coincides with $-2 \pi \alpha(\xi)$ if $\alpha=\beta$.

The preceding calculations imply that

$$
\omega_{\xi}=-2 \pi \sum_{\alpha \in \Phi_{+}} \alpha(\xi) x_{\alpha}^{*} \wedge y_{\alpha}^{*}
$$

where $\left\{x_{\alpha}^{*}\right\}_{\alpha \in \Phi_{+}} \cup\left\{y_{\alpha}^{*}\right\}_{\alpha \in \Phi_{+}}$is the basis of $\left(\mathfrak{t}^{\perp}\right)^{*}$ induced by our basis $\left\{x_{\alpha}\right\}_{\alpha \in \Phi_{+}} \cup\left\{y_{\alpha}\right\}_{\alpha \in \Phi_{+}}$of $\mathfrak{t}^{\perp}$. Now let $\gamma$ denote the wedge product of the bilinear forms $x_{\alpha}^{*} \wedge y_{\alpha}^{*}$, with $\alpha$ ranging over all $\alpha \in \Phi_{+}$. Equation (7) then implies that

$$
\Omega_{\xi}=(-2 \pi)^{m}\left(\prod_{\alpha \in \Phi_{+}} \alpha(\xi)\right) \gamma
$$

where $m$ is the number of positive roots. At the same time, analogous arguments establish that

$$
\Omega_{w \xi}=(-2 \pi)^{m}\left(\prod_{\alpha \in \Phi_{+}} \alpha(w \xi)\right) \gamma
$$

for all $w \in W$. It therefore suffices to prove that

$$
\prod_{\alpha \in \Phi_{+}} \alpha(w \xi)=(-1)^{\ell(w)} \prod_{\alpha \in \Phi_{+}} \alpha(\xi)
$$


We have

$$
\begin{aligned}
\prod_{\alpha \in \Phi_{+}} \alpha(w \xi) & =\prod_{\alpha \in \Phi_{+}}\left(w^{-1} \alpha\right)(\xi) \\
& =\prod_{\alpha \in w^{-1} \Phi_{+}} \alpha(\xi) \\
& =\left(\prod_{\alpha \in \Phi_{+} \cap w^{-1} \Phi_{+}} \alpha(\xi)\right)\left(\prod_{\alpha \in \Phi_{-} \cap w^{-1} \Phi_{+}} \alpha(\xi)\right) \\
& =\left(\prod_{\alpha \in \Phi_{+} \cap w^{-1} \Phi_{+}} \alpha(\xi)\right)\left(\prod_{\alpha \in \Phi_{+} \cap w^{-1} \Phi_{-}}(-\alpha(\xi))\right) \\
& =(-1)^{\ell(w)}\left(\prod_{\alpha \in \Phi_{+} \cap w^{-1} \Phi_{+}} \alpha(\xi)\right)\left(\prod_{\alpha \in \Phi_{+} \cap w^{-1} \Phi_{-}} \alpha(\xi)\right) \\
& =(-1)^{\ell(w)} \prod_{\alpha \in \Phi_{+}} \alpha(\xi),
\end{aligned}
$$

where the second-last line follows from the fact that $\ell(w)=\left|\Phi_{+} \cap w^{-1} \Phi_{-}\right|$. This completes the proof.

Fix $\xi \in \mathfrak{t}_{\text {reg }}$ and let $\mathcal{O} \subseteq \mathfrak{g}$ denote its adjoint orbit. Consider the unique $G$-equivariant diffeomorphism

$$
G / T \stackrel{\cong}{\longrightarrow}
$$

that sends $[e] \in G / T$ to $\xi \in \mathcal{O}$, and let $\Xi_{\xi}$ denote the pullback of $\Omega_{\mathcal{O}}$ along this diffeomorphism. It follows that $\Xi_{\xi}$ is a $G$-invariant volume form on $G / T$. Taking absolute values yields a density $\left|\Xi_{\xi}\right|$, whose square root is a half-density $\left|\Xi_{\xi}\right|^{\frac{1}{2}}$. Note that if $\xi, \xi^{\prime} \in \mathfrak{t}_{\text {reg }}$, then the product $\left|\Xi_{\xi}\right|^{\frac{1}{2}}\left|\Xi_{\xi^{\prime}}\right|^{\frac{1}{2}}$ is a density on $G / T$.

Lemma 3.2.2. Suppose that $\xi, \xi^{\prime} \in \mathfrak{t}$ are dominant and regular. The volume of $G / T$ with respect to $\left|\Xi_{\xi}\right|^{\frac{1}{2}}\left|\Xi_{\xi^{\prime}}\right|^{\frac{1}{2}}$ is

$$
\operatorname{Vol}\left(G / T,\left|\Xi_{\xi}\right|^{\frac{1}{2}}\left|\Xi_{\xi^{\prime}}\right|^{\frac{1}{2}}\right)=\frac{\left(\prod_{\alpha \in \Phi_{+}} \alpha(\xi) \alpha\left(\xi^{\prime}\right)\right)^{\frac{1}{2}}}{\prod_{\alpha \in \Phi_{+}}\langle\alpha, \rho\rangle},
$$

where $\rho:=\frac{1}{2} \sum_{\alpha \in \Phi_{+}} \alpha$.

Proof. Note that evaluating $\left|\Xi_{\xi}\right|^{\frac{1}{2}}$ (resp. $\left|\Xi_{\xi^{\prime}}\right|^{\frac{1}{2}}$ ) at $[e] \in G / T$ produces a half-density $\left(\left|\Xi_{\xi}\right|^{\frac{1}{2}}\right)_{[e]}$ (resp. $\left.\left(\left|\Xi_{\xi^{\prime}}\right|^{\frac{1}{2}}\right)_{[e]}\right)$ on $T_{[e]}(G / T)=\mathfrak{t}^{\perp}$. To describe these half-densities, we recall the notation used in the proof of Lemma 3.2.1. The arguments used to establish (8) then imply that

$$
\left(\left|\Xi_{\xi}\right|^{\frac{1}{2}}\right)_{[e]}=(2 \pi)^{\frac{m}{2}}\left(\prod_{\alpha \in \Phi_{+}} \alpha(\xi)\right)^{\frac{1}{2}}|\gamma|^{\frac{1}{2}} \quad \text { and } \quad\left(\left|\Xi_{\xi^{\prime}}\right|^{\frac{1}{2}}\right)_{[e]}=(2 \pi)^{\frac{m}{2}}\left(\prod_{\alpha \in \Phi_{+}} \alpha\left(\xi^{\prime}\right)\right)^{\frac{1}{2}}|\gamma|^{\frac{1}{2}} \text {. }
$$

The value of $\left|\Xi_{\xi}\right|^{\frac{1}{2}}\left|\Xi_{\xi^{\prime}}\right|^{\frac{1}{2}}$ at $[e]$ is therefore given by

$$
\left(\left|\Xi_{\xi}\right|^{\frac{1}{2}}\left|\Xi_{\xi^{\prime}}\right|^{\frac{1}{2}}\right)_{[e]}=(2 \pi)^{m}\left(\prod_{\alpha \in \Phi_{+}} \alpha(\xi) \alpha\left(\xi^{\prime}\right)\right)^{\frac{1}{2}}|\gamma| .
$$


Now consider the unique $G$-invariant Riemannian metric on $G / T$ that coincides with $\langle\cdot, \cdot\rangle$ on $T_{[e]}(G / T)=\mathfrak{t}^{\perp}$, and write $\theta$ for the $G$-invariant density on $G / T$ induced by this metric. Let us also recall that $\left\{x_{\alpha}\right\}_{\alpha \in \Phi_{+}} \cup\left\{y_{\alpha}\right\}_{\alpha \in \Phi_{+}}$is an orthonormal basis of $\mathfrak{t}^{\perp}$ with respect to $\langle\cdot, \cdot\rangle$, and that $\gamma$ is the wedge product of all the skew-symmetric bilinear forms $x_{\alpha}^{*} \wedge y_{\alpha}^{*}$. This last sentence implies that $\theta_{[e]}=|\gamma|$, where $\theta_{[e]}$ denotes the value of $\theta$ at $[e]$. The statement (9) then becomes

$$
\left(\left|\Xi_{\xi}\right|^{\frac{1}{2}}\left|\Xi_{\xi^{\prime}}\right|^{\frac{1}{2}}\right)_{[e]}=(2 \pi)^{m}\left(\prod_{\alpha \in \Phi_{+}} \alpha(\xi) \alpha\left(\xi^{\prime}\right)\right)^{\frac{1}{2}} \theta_{[e]} .
$$

Since $\left|\Xi_{\xi}\right|^{\frac{1}{2}}\left|\Xi_{\xi^{\prime}}\right|^{\frac{1}{2}}$ and $\theta$ are $G$-invariant, it follows that

$$
\left|\Xi_{\xi}\right|^{\frac{1}{2}}\left|\Xi_{\xi^{\prime}}\right|^{\frac{1}{2}}=(2 \pi)^{m}\left(\prod_{\alpha \in \Phi_{+}} \alpha(\xi) \alpha\left(\xi^{\prime}\right)\right)^{\frac{1}{2}} \theta .
$$

We conclude that

$$
\operatorname{Vol}\left(G / T,\left|\Xi_{\xi}\right|^{\frac{1}{2}}\left|\Xi_{\xi^{\prime}}\right|^{\frac{1}{2}}\right)=(2 \pi)^{m}\left(\prod_{\alpha \in \Phi_{+}} \alpha(\xi) \alpha\left(\xi^{\prime}\right)\right)^{\frac{1}{2}} \operatorname{Vol}(G / T, \theta),
$$

where $\operatorname{Vol}(G / T, \theta)$ is the volume of $G / T$ with respect to $\theta$. At the same time, Equation (20) in [13] implies that

$$
\operatorname{Vol}(G / T, \theta)=\frac{1}{(2 \pi)^{m} \prod_{\alpha \in \Phi_{+}}\langle\alpha, \rho\rangle} .
$$

The desired result now follows from (10) and (11).

3.3. Haar measures on maximal tori. Let $T^{\prime} \subseteq G$ be any maximal torus, possibly different from the maximal torus $T$ fixed throughout this manuscript. Write $\Theta_{T^{\prime}} \in \Omega^{r}\left(T^{\prime}\right)$ for the left-invariant $r$-form that induces the Haar measure on $T^{\prime}$. The value of $\Theta_{T^{\prime}}$ at the identity is then a vector

$$
\left(\Theta_{T^{\prime}}\right)_{e} \in \wedge^{r}\left(\left(\mathfrak{t}^{\prime}\right)^{*}\right)
$$

where $\mathfrak{t}^{\prime}$ is the Lie algebra of $T^{\prime}$.

Proposition 3.3.1. Suppose that $T^{\prime}, T^{\prime \prime} \subseteq G$ are maximal tori with respective Lie algebras $\mathfrak{t}^{\prime}, \mathfrak{t}^{\prime \prime} \subseteq \mathfrak{g}$. If $\left\{\zeta_{1}^{\prime}, \ldots, \zeta_{r}^{\prime}\right\}$ and $\left\{\zeta_{1}^{\prime \prime}, \ldots, \zeta_{r}^{\prime \prime}\right\}$ are orthonormal bases of $\mathfrak{t}^{\prime}$ and $\mathfrak{t}^{\prime \prime}$, respectively, then

$$
\left|\left(\Theta_{T^{\prime}}\right)_{e}\left(\zeta_{1}^{\prime}, \ldots, \zeta_{r}^{\prime}\right)\right|=\left|\left(\Theta_{T^{\prime \prime}}\right)_{e}\left(\zeta_{1}^{\prime \prime}, \ldots, \zeta_{r}^{\prime \prime}\right)\right| .
$$

Proof. Choose an element $g \in G$ satisfying $T^{\prime \prime}=g T^{\prime} g^{-1}$. It follows that conjugation by $g$ defines a Lie group isomorphism $T^{\prime} \stackrel{\cong}{\longrightarrow} T^{\prime \prime}$, so that

$$
\left(\Theta_{T^{\prime}}\right)_{e}\left(\zeta_{1}^{\prime}, \ldots, \zeta_{r}^{\prime}\right)=\left(\Theta_{T^{\prime \prime}}\right)_{e}\left(\operatorname{Ad}_{g}\left(\zeta_{1}^{\prime}\right), \ldots, \operatorname{Ad}_{g}\left(\zeta_{r}^{\prime}\right)\right) .
$$

We also note that $\left\{\operatorname{Ad}_{g}\left(\zeta_{1}^{\prime}\right), \ldots, \operatorname{Ad}_{g}\left(\zeta_{r}^{\prime}\right)\right\}$ is an orthonormal basis of $\mathfrak{t}^{\prime \prime}$, owing to the fact that $\langle\cdot, \cdot\rangle$ is $G$-invariant. The bases $\left\{\operatorname{Ad}_{g}\left(\zeta_{1}^{\prime}\right), \ldots, \operatorname{Ad}_{g}\left(\zeta_{r}^{\prime}\right)\right\}$ and $\left\{\zeta_{1}^{\prime \prime}, \ldots, \zeta_{r}^{\prime \prime}\right\}$ are therefore related by a linear automorphism $\mathfrak{t}^{\prime \prime} \longrightarrow \mathfrak{t}^{\prime \prime}$ having determinant 1 or -1 , implying that

$$
\left|\left(\Theta_{T^{\prime \prime}}\right)_{e}\left(\operatorname{Ad}_{g}\left(\zeta_{1}^{\prime}\right), \ldots, \operatorname{Ad}_{g}\left(\zeta_{r}^{\prime}\right)\right)\right|=\left|\left(\Theta_{T^{\prime \prime}}\right)_{e}\left(\zeta_{1}^{\prime \prime}, \ldots, \zeta_{r}^{\prime \prime}\right)\right| .
$$

The desired result now follows from (12) and (13). 
One consequence is that the positive real number

$$
\kappa(G):=\left|\left(\Theta_{T^{\prime}}\right)_{e}\left(\zeta_{1}^{\prime}, \ldots, \zeta_{r}^{\prime}\right)\right|
$$

does not depend on the choice of maximal torus $T^{\prime} \subseteq G$ and orthonormal basis $\left\{\zeta_{1}^{\prime}, \ldots, \zeta_{r}^{\prime}\right\}$ of the Lie algebra of $T^{\prime}$.

3.4. The phase function on $\Lambda_{\mathcal{O}}$. Let $\mathcal{O} \subseteq \mathfrak{g}$ be a regular, integral adjoint orbit and recall the Lagrangian submanifold $\Lambda_{\mathcal{O}} \subseteq T^{*} G$ from Section [2.1. Note that if $(g, \xi) \in \Lambda_{\mathcal{O}}$, then $G_{\xi}$ is a maximal torus in $G$. It follows that the exponential map exp : $\mathfrak{g} \longrightarrow G$ restricts to a surjective group morphism $\mathfrak{g}_{\xi} \longrightarrow G_{\xi}$. We may therefore choose $\eta \in \mathfrak{g}_{\xi}$ satisfying $\exp (\eta)=g$ and define

$$
\psi_{\mathcal{O}}(g, \xi):=e^{2 \pi i\langle\xi, \eta\rangle} \in S^{1} \subseteq \mathbb{C} .
$$

Our integrality hypotheses implies that $\psi_{\mathcal{O}}(g, \xi)$ is independent of $\eta$, and we thereby obtain a well-defined map

$$
\psi_{\mathcal{O}}: \Lambda_{\mathcal{O}} \longrightarrow S^{1}
$$

Guillemin and Sternberg show $\psi_{\mathcal{O}}$ to be a phase function for the Lagrangian submanifold $\Lambda_{\mathcal{O}} \subseteq T^{*} G$.

Now recall the $G$-action on $T^{*} G$ defined in (5). This action leaves $\Lambda_{\mathcal{O}}$ invariant, in which context we have the following result.

Lemma 3.4.1. If $\mathcal{O} \subseteq \mathfrak{g}$ is a regular, integral adjoint orbit, then the phase function $\psi_{\mathcal{O}}: \Lambda_{\mathcal{O}} \longrightarrow S^{1}$ is $G$-invariant.

Proof. Suppose that $(g, \xi) \in \Lambda_{\mathcal{O}}$ and $h \in G$. Choose $\eta \in \mathfrak{g}_{\xi}$ for which $g=\exp (\eta)$, and consider the vectors $\xi^{\prime}=\operatorname{Ad}_{h}(\xi)$ and $\eta^{\prime}=\operatorname{Ad}_{h}(\eta)$. We then have $\eta^{\prime} \in \mathfrak{g}_{\xi^{\prime}}$ and $\exp \left(\eta^{\prime}\right)=h g h^{-1}$. It follows that

$$
\begin{aligned}
\psi_{\mathcal{O}}(h \cdot(g, \xi)) & =\psi_{\mathcal{O}}\left(h g h^{-1}, \xi^{\prime}\right) \\
& =e^{2 \pi i\left\langle\xi^{\prime}, \eta^{\prime}\right\rangle} \\
& =e^{2 \pi i\langle\xi, \eta\rangle} \\
& =\psi_{\mathcal{O}}(g, \xi),
\end{aligned}
$$

where the second-last line is a consequence of $\langle\cdot, \cdot\rangle$ being $G$-invariant. This completes the proof.

3.5. The half-density on $\Lambda_{\mathcal{O}}$. We now describe the half-density on $\Lambda_{\mathcal{O}}$ constructed in [6], where $\mathcal{O} \subseteq \mathfrak{g}$ is a regular adjoint orbit. To this end, recall the $G$-action on $\Lambda_{\mathcal{O}}$ mentioned in Section 3.4. Let $G(g, \xi) \subseteq \Lambda_{\mathcal{O}}$ denote the $G$-orbit of $(g, \xi) \in \Lambda_{\mathcal{O}}$; this is consistent with the notation for $G$-orbits explained in Section 3.1 .

Lemma 3.5.1. If $\mathcal{O} \subseteq \mathfrak{g}$ is a regular adjoint orbit, then

$$
T_{(g, \xi)} \Lambda_{\mathcal{O}}=T_{(g, \xi)}(G(g, \xi)) \oplus T_{(g, \xi)}\left(G_{\xi} \times\{\xi\}\right)
$$

for all $(g, \xi) \in \Lambda_{\mathcal{O}}$. The $G$-action on $\Lambda_{\mathcal{O}}$ respects these tangent space decompositions.

Proof. Since $\mathcal{O}$ is a regular, the group $G_{\xi}$ is abelian. A straightforward calculation then reveals that $G_{\xi}$ is the $G$-stabilizer of $(g, \xi) \in \Lambda_{\mathcal{O}}$. It follows that $T_{(g, \xi)}(G(g, \xi))$ has dimension $n-\operatorname{dim}\left(G_{\xi}\right)$, while we see that $T_{(g, \xi)}\left(G_{\xi} \times\{\xi\}\right)$ has dimension $\operatorname{dim}\left(G_{\xi}\right)$. We also know $T_{(g, \xi)} \Lambda_{\mathcal{O}}$ to have dimension $n$, as $\Lambda_{\mathcal{O}}$ is a Lagrangian submanifold of $T^{*} G$. In light of these last two sentences,

$$
T_{(g, \xi)} \Lambda_{\mathcal{O}}=T_{(g, \xi)}(G(g, \xi)) \oplus T_{(g, \xi)}\left(G_{\xi} \times\{\xi\}\right)
$$

holds if and only if

$$
T_{(g, \xi)}(G(g, \xi)) \cap T_{(g, \xi)}\left(G_{\xi} \times\{\xi\}\right)=\{0\} .
$$


Note that each vector in $T_{(g, \xi)}(G(g, \xi))$ has the form $\left(\left(\eta_{G}\right)_{g},\left(\eta_{\mathcal{O}}\right)_{\xi}\right)$ for $\eta \in \mathfrak{g}$, where $\eta_{G}$ (resp. $\eta_{\mathcal{O}}$ ) denotes the fundamental vector field for $\eta$ with respect to the conjugation action (resp. adjoint action) of $G$ on $G$ (resp. $\mathcal{O}$ ). With this in mind, let $\eta \in \mathfrak{g}$ be such that

$$
\left(\left(\eta_{G}\right)_{g},\left(\eta_{\mathcal{O}}\right)_{\xi}\right) \in T_{(g, \xi)}\left(G_{\xi} \times\{\xi\}\right) .
$$

It follows that $\left(\eta_{\mathcal{O}}\right)_{\xi}=0$, while a straightforward calculation reveals that $\left(\eta_{\mathcal{O}}\right)_{\xi}=[\eta, \xi]$. We conclude that $\eta \in \mathfrak{g}_{\xi}$. Since $G_{\xi}$ is abelian and contains $g$, this is easily seen to imply that $\left(\eta_{G}\right)_{g}=0$. Hence

$$
\left(\left(\eta_{G}\right)_{g},\left(\eta_{\mathcal{O}}\right)_{\xi}\right)=(0,0)
$$

and one deduces that $T_{(g, \xi)}(G(g, \xi))$ and $T_{(g, \xi)}\left(G_{\xi} \times\{\xi\}\right)$ intersect trivially. In light of the previous paragraph, we have

$$
T_{(g, \xi)} \Lambda_{\mathcal{O}}=T_{(g, \xi)}(G(g, \xi)) \oplus T_{(g, \xi)}\left(G_{\xi} \times\{\xi\}\right) .
$$

It remains to prove that the $G$-action on $\Lambda_{\mathcal{O}}$ respects our tangent space decompositions. We thus suppose that $(g, \xi) \in \Lambda_{\mathcal{O}}$ and $h \in G$, and we set

$$
\left(g^{\prime}, \xi^{\prime}\right):=\left(h g h^{-1}, \operatorname{Ad}_{h}(\xi)\right) .
$$

Our task is to verify that the action of $h$ sends $T_{(g, \xi)}(G(g, \xi))$ and $T_{(g, \xi)}\left(G_{\xi} \times\{\xi\}\right)$ to $T_{\left(g^{\prime}, \xi^{\prime}\right)}\left(G\left(g^{\prime}, \xi^{\prime}\right)\right)$ and $T_{\left(g^{\prime}, \xi^{\prime}\right)}\left(G_{\xi^{\prime}} \times\left\{\xi^{\prime}\right\}\right)$, respectively. But this follows from the observation that $h$ sends $G(g, \xi)$ and $G_{\xi} \times\{\xi\}$ to $G\left(g^{\prime}, \xi^{\prime}\right)$ and $h G_{\xi} h^{-1} \times\left\{\operatorname{Ad}_{h}(\xi)\right\}=G_{\xi^{\prime}} \times\left\{\xi^{\prime}\right\}$, respectively.

This result has the following immediate implication: if one has a half-density on each of $T_{(g, \xi)}(G(g, \xi))$ and $T_{(g, \xi)}\left(G_{\xi} \times\{\xi\}\right)$, then one also has a half-density on $T_{(g, \xi)} \Lambda_{\mathcal{O}}$ (see (48)). This principle gives rise to a half-density $\rho_{\mathcal{O}}$ on $\Lambda_{\mathcal{O}}$, as we now explain.

Let $\mathcal{O} \subseteq \mathfrak{g}$ be a regular adjoint orbit and fix $(g, \xi) \in \Lambda_{\mathcal{O}}$. Recall that $G_{\xi}$ is the $G$-stabilizer of $(g, \xi)$ in $\Lambda_{\mathcal{O}}$, as explained in the proof above. One straightforward consequence is that

$$
G(g, \xi) \longrightarrow \mathcal{O}, \quad(h, \eta) \mapsto \eta
$$

defines a $G$-equivariant diffeomorphism. This diffeomorphism identifies $T_{(g, \xi)}(G(g, \xi))$ with $T_{\xi} \mathcal{O}$, and we recall that the symplectic structure on $\mathcal{O}$ induces a half-density $\left|\Omega_{\xi}\right|^{\frac{1}{2}}$ on $T_{\xi} \mathcal{O} \cong \mathfrak{g}_{\xi}^{\perp}$. The tangent space $T_{(g, \xi)}(G(g, \xi))$ thereby inherits a half-density. We also have an obvious identification of $T_{(g, \xi)}\left(G_{\xi} \times\{\xi\}\right)$ with $T_{g} G_{\xi}$, and the Haar measure on $G_{\xi}$ induces a half-density $\left|\left(\Theta_{G_{\xi}}\right)_{g}\right|^{\frac{1}{2}}$ on $T_{g} G_{\xi}$ (see Section 3.3). These considerations produce a half-density on $T_{(g, \xi)}\left(G_{\xi} \times\{\xi\}\right.$ ), and it combines with that on $T_{(g, \xi)}(G(g, \xi))$ to yield a half-density on the direct $\operatorname{sum} T_{(g, \xi)}(G(g, \xi)) \oplus T_{(g, \xi)}\left(G_{\xi} \times\{\xi\}\right)=$ $T_{(g, \xi)} \Lambda_{\mathcal{O}}$ (see (48)). This new half-density is precisely $\rho_{\mathcal{O}}$ at the point $(g, \xi)$.

This next result uses the notation and discussion in Sections 3.2 and 3.3 .

Proposition 3.5.2. Let $\mathcal{O} \subseteq \mathfrak{g}$ be a regular adjoint orbit and suppose that $(g, \xi) \in \Lambda_{\mathcal{O}}$. Suppose also that $\left\{\eta_{1}, \ldots, \eta_{n-r}\right\}$ and $\left\{\zeta_{1}, \ldots, \zeta_{r}\right\}$ are bases of $\mathfrak{g}_{\xi}^{\perp}$ and $\mathfrak{g}_{\xi}$, respectively. Write $\overline{\eta_{j}}$ for the fundamental vector field on $T^{*} G$ associated to $\eta_{j}$ via the $G$-action (5), i.e.

$$
\overline{\eta_{j}}:=\left(\eta_{j}\right)_{T * G}
$$

for all $j \in\{1, \ldots, n-r\}$. Let us also define the vector

$$
\left(\widehat{\zeta_{k}}\right)_{(g, \xi)}:=\left(\left(d L_{g}\right)_{e}\left(\zeta_{k}\right), 0\right) \in T_{g} G_{\xi} \oplus\{0\}=T_{(g, \xi)}\left(G_{\xi} \times\{\xi\}\right) \subseteq T_{(g, \xi)} \Lambda_{\mathcal{O}}
$$

for each $k \in\{1, \ldots, r\}$, where $L_{g}: G \longleftrightarrow G$ denotes left translation by $g$. The following statements then hold. 
(i) The sets

$$
\left\{\left(\overline{\eta_{1}}\right)_{(g, \xi)}, \ldots,\left(\overline{\eta_{n-r}}\right)_{(g, \xi)}\right\} \quad \text { and } \quad\left\{\left(\widehat{\zeta_{1}}\right)_{(g, \xi)}, \ldots,\left(\widehat{\zeta_{r}}\right)_{(g, \xi)}\right\}
$$

are bases of $T_{(g, \xi)}(G(g, \xi))$ and $T_{(g, \xi)}\left(G_{\xi} \times\{\xi\}\right)$, respectively.

(ii) The set

is a basis of $T_{(g, \xi)} \Lambda_{\mathcal{O}}$.

$$
\left\{\left(\overline{\eta_{1}}\right)_{(g, \xi)}, \ldots,\left(\overline{\eta_{n-r}}\right)_{(g, \xi)},\left(\widehat{\zeta_{1}}\right)_{(g, \xi)}, \ldots,\left(\widehat{\zeta_{r}}\right)_{(g, \xi)}\right\}
$$

(iii) The value of $\left(\rho_{\mathcal{O}}\right)_{(g, \xi)}$ on this basis is

$$
\kappa(G)^{\frac{1}{2}} \cdot\left|\Omega_{\xi}\left(\eta_{1}, \ldots, \eta_{n-r}\right)\right|^{\frac{1}{2}} .
$$

Proof. We begin by proving (i). To this end, recall that the isomorphism

$$
T_{(g, \xi)}(G(g, \xi)) \stackrel{\cong}{\longrightarrow} T_{\xi} \mathcal{O}
$$

obtained by differentiating (15) at $\xi$. This isomorphism sends $\left(\overline{\eta_{1}}\right)_{(g, \xi)}, \ldots,\left(\overline{\eta_{n-r}}\right)_{(g, \xi)}$ to $\eta_{1}, \ldots, \eta_{n-r} \in$ $\mathfrak{g}_{\xi}^{\perp} \cong T_{\xi} \mathcal{O}$, respectively. It follows that $\left\{\left(\overline{\eta_{1}}\right)_{(g, \xi)}, \ldots,\left(\overline{\eta_{n-r}}\right)_{(g, \xi)}\right\}$ is a basis of $T_{(g, \xi)}(G(g, \xi))$. On the other hand, $\left\{\zeta_{1}, \ldots, \zeta_{r}\right\}$ being a basis of $\mathfrak{g}_{\xi}$ implies that $\left\{\left(d L_{g}\right)_{e}\left(\zeta_{1}\right), \ldots,\left(d L_{g}\right)_{e}\left(\zeta_{r}\right)\right\}$ is a basis of $T_{g} G_{\xi}$. Under the obvious identification

$$
T_{g} G_{\xi} \cong T_{(g, \xi)}\left(G_{\xi} \times\{\xi\}\right),
$$

this basis corresponds to the ordered set

$$
\left\{\left(\widehat{\zeta_{1}}\right)_{(g, \xi)}, \ldots,\left(\widehat{\zeta}_{r}\right)_{(g, \xi)}\right\} \subseteq T_{(g, \xi)}\left(G_{\xi} \times\{\xi\}\right) .
$$

We conclude that $\left\{\left(\widehat{\zeta}_{1}\right)_{(g, \xi)}, \ldots,\left(\widehat{\zeta}_{r}\right)_{(g, \xi)}\right\}$ is a basis of $T_{(g, \xi)}\left(G_{\xi} \times\{\xi\}\right)$, completing our proof of (i).

Part (ii) follows from (i) and Lemma 3.5.1. To prove (iii), recall our proof of (i) and the definition of $\left(\rho_{\mathcal{O}}\right)_{(g, \xi)}$ given in the paragraph preceding this proposition. These considerations imply that $\left(\rho_{\mathcal{O}}\right)_{(g, \xi)}$ takes the value

$$
\left|\Omega_{\xi}\left(\eta_{1}, \ldots, \eta_{n-r}\right)\right|^{\frac{1}{2}}\left|\left(\Theta_{G_{\xi}}\right)_{g}\left(\left(d L_{g}\right)_{e}\left(\zeta_{1}\right), \ldots,\left(d L_{g}\right)_{e}\left(\zeta_{r}\right)\right)\right|^{\frac{1}{2}}
$$

on the indicated basis.

We have the following additional property of $\rho_{\mathcal{O}}$.

Proposition 3.5.3. If $\mathcal{O} \subseteq \mathfrak{g}$ is a regular adjoint orbit, then $\rho_{\mathcal{O}}$ is invariant under the $G$-action on $\Lambda_{\mathcal{O}}$.

Proof. Suppose that $(g, \xi) \in \Lambda_{\mathcal{O}}$ and $h \in G$, and set

$$
\left(g^{\prime}, \xi^{\prime}\right):=\left(h g h^{-1}, \operatorname{Ad}_{h}(\xi)\right) .
$$

We now make three remarks. The first requires us to equip $T_{g} G_{\xi}$ and $T_{g^{\prime}} G_{\xi^{\prime}}$ with the half-densities arising from the Haar measures on $G_{\xi}$ and $G_{\xi^{\prime}}$, respectively. Since conjugation by $h$ defines a group isomorphism from $G_{\xi}$ to $G_{\xi^{\prime}}$, it identifies the half-density on $T_{g^{\prime}} G_{\xi^{\prime}}$ with that on $T_{g} G_{\xi}$. To begin our second remark, we note that $h$ preserves the symplectic form on $\mathcal{O}$. It follows that $h$ identifies the half-density on $T_{\xi^{\prime}} \mathcal{O}$ with that on $T_{\xi} \mathcal{O}$, where these two half-densities are induced by the symplectic form on $\mathcal{O}$. Our final remark is that $h$ respects the tangent space decompositions (14), a consequence of Lemma 3.5.1. By the description of $\rho_{\mathcal{O}}$ given in Proposition 3.5.2(iii), these three remarks show $h$ to preserve $\rho_{\mathcal{O}}$. We conclude that $\rho_{\mathcal{O}}$ is $G$-invariant.

\section{Some quasi-Hamiltonian geometry}

We now discuss the salient parts of quasi-Hamiltonian geometry [1, emphasizing that of the universal centralizers $\Lambda_{\mathcal{C}}$. 
4.1. Quasi-Hamiltonian $G$-spaces. Suppose that $M$ is a $G$-manifold and write $g \cdot m \in M$ for the action of $g \in G$ on a point $m \in M$. Each $\xi \in \mathfrak{g}$ then determines a fundamental vector field $\xi_{M}$ on $M$, defined by

$$
\left(\xi_{M}\right)_{m}:=\left.\frac{d}{d t}\right|_{t=0}(\exp (t \xi) \cdot m), \quad m \in M
$$

Now let $M$ be an arbitrary manifold and consider $\Omega^{1}(M ; \mathfrak{g})$, the real vector space of $\mathfrak{g}$-valued differential 1-forms on $M$. We have a bilinear pairing

$$
(\cdot, \cdot): \Omega^{1}(M ; \mathfrak{g}) \otimes_{\mathbb{R}} \Omega^{1}(M ; \mathfrak{g}) \longrightarrow \Omega^{2}(M)
$$

defined by

$$
(\alpha, \beta)_{m}\left(v_{1}, v_{2}\right)=\left\langle\alpha_{m}\left(v_{1}\right), \beta_{m}\left(v_{2}\right)\right\rangle-\left\langle\alpha_{m}\left(v_{2}\right), \beta_{m}\left(v_{1}\right)\right\rangle
$$

for all $\alpha, \beta \in \Omega^{1}(M ; \mathfrak{g}), m \in M$, and $v_{1}, v_{2} \in T_{m} M$. In the case $M=G$, we let $\theta^{L}, \theta^{R} \in \Omega^{1}(G ; \mathfrak{g})$ denote the left and right-invariant Maurer-Cartan forms, respectively. One then has

$$
\left(\theta^{L}\right)_{g}\left(\left(d L_{g}\right)_{e}(\xi)\right)=\xi \quad \text { and } \quad\left(\theta^{R}\right)_{g}\left(\left(d R_{g}\right)_{e}(\xi)\right)=\xi
$$

for all $g \in G$ and $\xi \in \mathfrak{g}$, where $L_{g}: G \longrightarrow G$ and $R_{g}: G \longrightarrow G$ are left and right translation by $g$, respectively.

Definition 4.1.1. A quasi-Hamiltonian $G$-space consists of a $G$-manifold $M$, a $G$-invariant 2 -form $\omega \in \Omega^{2}(M)$, and a smooth map $\mu: M \longrightarrow G$, subject to the following conditions:

(i) $d \omega=-\mu^{*} \chi$, where $\chi \in \Omega^{3}(G)$ is the Cartan 3-form;

(ii) $\iota_{\xi_{M}} \omega=\frac{1}{2}\left\langle\theta^{L}+\theta^{R}, \xi\right\rangle$;

(iii) $\operatorname{ker}\left(\omega_{m}\right)=\left\{\left(\xi_{M}\right)_{m}: \xi \in \operatorname{ker}\left(\operatorname{Ad}_{\mu(m)}+\operatorname{id}_{\mathfrak{g}}\right)\right\}$ for all $m \in M$;

(iv) $\mu$ is $G$-equivariant with respect to the given action on $M$ and the conjugation action on $G$. One refers to $\mu$ and $\omega$ as the moment map and quasi-Hamiltonian form, respectively.

Suppose that this definition is satisfied and write

$$
M^{\circ}:=\left\{m \in M: \operatorname{ker}\left(\omega_{m}\right)=\{0\}\right\}
$$

for the open, $G$-invariant, even-dimensional submanifold of $M$ on which $\omega$ is non-degenerate. Note that $\omega$ need not restrict to a symplectic form on $M^{\circ}$; this restricted form need not be closed. Nevertheless, $\left(T_{m} M, \omega_{m}\right)$ is a symplectic vector space for all $m \in M^{\circ}$.

Remark 4.1.2. We also observe that $\mu^{-1}(e) \subseteq M^{\circ}$, as follows from the condition (iii).

Definition 4.1.3. Consider the quasi-Hamiltonian $G$-space described in Definition 4.1.1. Let $L \subseteq$ $M$ be a submanifold with inclusion map $j: L \longrightarrow M$. We call $L$ an isotropic submanifold of $M$ if $j^{*} \omega=0$. If $L \subseteq M^{\circ}$ and $\operatorname{dim}(L)=\frac{1}{2} \operatorname{dim}\left(M^{\circ}\right)$ also hold, we call $L$ a quasi-Hamiltonian Lagrangian submanifold of $M$.

4.2. The internally fused double. Examples of quasi-Hamiltonian $G$-spaces include the so-called internally fused double $D(G)$, a construction described in [1]. To obtain it, one first notes that $D(G)=G \times G$ as a manifold. Now let $G$ act on $D(G)$ via

$$
g \cdot(h, k):=\left(g h g^{-1}, g k g^{-1}\right), \quad g \in G,(h, k) \in D(G) .
$$

The map

$$
\mu_{D(G)}: D(G) \longrightarrow G, \quad(g, h) \mapsto g h g^{-1} h^{-1}, \quad(g, h) \in D(G)
$$

is then $G$-equivariant with respect to the conjugation action on $G$. Let us also consider the two-form $\omega_{D(G)} \in \Omega^{2}(D(G))$ defined by

$$
\omega_{D(G)}:=\frac{1}{2}\left(\pi_{1}^{*} \theta^{L}, \pi_{2}^{*} \theta^{R}\right)+\frac{1}{2}\left(\pi_{1}^{*} \theta^{R}, \pi_{2}^{*} \theta^{L}\right)+\frac{1}{2}\left(\left(\pi_{1} \pi_{2}\right)^{*} \theta^{L},\left(\pi_{1}^{-1} \pi_{2}^{-1}\right)^{*} \theta^{R}\right),
$$


where $\pi_{1}, \pi_{2}: D(G) \longrightarrow G$ are the projections onto the first and second factors, respectively, and $\pi_{1} \pi_{2}, \pi_{1}^{-1} \pi_{2}^{-1}: D(G) \longrightarrow G$ are defined by

$$
\left(\pi_{1} \pi_{2}\right)(g, h)=g h, \quad\left(\pi_{1}^{-1} \pi_{2}^{-1}\right)(g, h)=g^{-1} h^{-1}, \quad(g, h) \in D(G) .
$$

A straightforward calculation shows $\omega$ to be $G$-invariant, an instance of the following more substantial result (cf. [1, Example 6.1]).

Proposition 4.2.1. The $G$-manifold $D(G)$, two-form $\omega_{D(G)}$, and map $\mu_{D(G)}$ constitute a quasiHamiltonian G-space.

One calls this quasi-Hamiltonian $G$-space the internally fused double of $G$.

4.3. The gauge-theoretic realization of $D(G)$. We now recall a gauge-theoretic realization of $D(G)$ described in [1]. The reader will find a more comprehensive account in Section 9 of the aforementioned reference.

Let $\Sigma$ denote a one-holed torus and fix a real number $\lambda>1$. Write $\mathcal{A}_{\text {flat }}(\Sigma)$ for the Banach manifold of flat connections on the trivial principal $G$-bundle $\Sigma \times G \longrightarrow \Sigma$ of Sobolev class $\lambda$, and write $\mathcal{G}(\Sigma)$ for the Banach Lie group of all maps $\Sigma \longrightarrow G$ having Sobolev class $\lambda+1$. This group acts on $\mathcal{A}_{\text {flat }}(\Sigma)$ in a canonical way (see [1, Section 9.1]), and one calls it the gauge group. Now fix a point $x$ on the boundary of $\Sigma$, and consider the restricted gauge group

$$
\mathcal{G}(\Sigma)_{\text {res }}:=\{f \in \mathcal{G}(\Sigma): f(x)=e\} .
$$

This subgroup of $\mathcal{G}(\Sigma)$ acts freely on $\mathcal{A}_{\text {flat }}(\Sigma)$, and the quotient

$$
\mathcal{M}(\Sigma):=\mathcal{A}_{\text {flat }}(\Sigma) / G(\Sigma)_{\text {res }}
$$

is a finite-dimensional manifold. The action of $\mathcal{G}(\Sigma)$ on $\mathcal{A}_{\text {flat }}(\Sigma)$ descends to an action of $\mathcal{G}(\Sigma) / \mathcal{G}(\Sigma)_{\text {res }} \cong$ $G$ on $M(\Sigma)$. One then has the following specialized version of [1, Theorem 9.1].

Theorem 4.3.1. The G-manifold $\mathcal{M}(\Sigma)$ is a quasi-Hamiltonian G-space in a canonical way.

Now choose loops $\gamma_{1}, \gamma_{2}:[0,1] \longrightarrow \Sigma$ at $x$ that satisfy the following properties:

(i) the images of $\gamma_{1}$ and $\gamma_{2}$ intersect only at $x$;

(ii) the homotopy classes $\left[\gamma_{1}\right]$ and $\left[\gamma_{2}\right]$ freely generate $\pi_{1}(\hat{\Sigma}, x)$ as an abelian group, where $\hat{\Sigma}$ is the torus obtained by capping off the boundary of $\Sigma$;

(iii) we have $\left[\gamma_{1}\right]\left[\gamma_{2}\right]\left[\gamma_{1}\right]^{-1}\left[\gamma_{2}\right]^{-1}=[\delta]$ in $\pi_{1}(\Sigma, x)$, where $[\delta]$ is a generator of $\pi_{1}(\partial \Sigma, x)$.

These choices give rise to a well-defined map

$$
\Phi_{\gamma_{1}, \gamma_{2}}: M(\Sigma) \longrightarrow D(G), \quad[\nabla] \mapsto\left(\operatorname{Hol}_{\gamma_{1}}(\nabla), \operatorname{Hol}_{\gamma_{2}}(\nabla)\right), \quad[\nabla] \in \mathcal{M}(\Sigma),
$$

where $\operatorname{Hol}_{\gamma_{1}}(\nabla), \operatorname{Hol}_{\gamma_{2}}(\nabla) \in G$ are the holonomies of $\nabla \in \mathcal{A}_{\text {flat }}(\Sigma)$ around $\gamma_{1}$ and $\gamma_{2}$, respectively. This leads to the following special case of [1, Theorem 9.3].

Theorem 4.3.2. Let $\gamma_{1}, \gamma_{2}:[0,1] \longrightarrow \Sigma$ be as described above, and equip $\mathcal{M}(\Sigma)$ with the quasiHamiltonian $G$-space structure from Theorem 4.3.1. The map $\Phi_{\gamma_{1}, \gamma_{2}}: \mathcal{M}(\Sigma) \longrightarrow D(G)$ is then an isomorphism of quasi-Hamiltonian $G$-spaces.

Now let $\operatorname{Diff}^{+}(\Sigma)$ denote the group of all orientation-preserving diffeomorphisms $\Sigma \longrightarrow \Sigma$, endowed with the $C^{1}$-topology. Consider the subgroup

$$
\operatorname{Diff}^{+}(\Sigma)_{\mathrm{res}}:=\left\{\phi \in \operatorname{Diff}^{+}(\Sigma): \phi(x)=x\right\}
$$

and its identify component $\left(\operatorname{Diff}^{+}(\Sigma)_{\text {res }}\right)^{\circ}$. The quotient group

$$
\Gamma(\Sigma):=\operatorname{Diff}^{+}(\Sigma)_{\mathrm{res}} /\left(\operatorname{Diff}^{+}(\Sigma)_{\mathrm{res}}\right)^{\circ}
$$


is then called the mapping class group. One has a well-defined action of $\Gamma(\Sigma)$ on $\mathcal{M}(\Sigma)$ given by

$$
[\phi] \cdot[\nabla]:=\left[\left(\phi^{-1}\right)^{*} \nabla\right], \quad[\phi] \in \Gamma(\Sigma),[\nabla] \in \mathcal{M}(\Sigma),
$$

where $\left(\phi^{-1}\right)^{*} \nabla$ denotes the pullback of $\nabla$ along the diffeomorphism $\phi^{-1}: \Sigma \longrightarrow \Sigma$. This gives context for the following result, which is explained in [1, Section 9.4].

Proposition 4.3.3. The above-defined action of $\Gamma(\Sigma)$ respects the quasi-Hamiltonian $G$-space structure on $\mathcal{M}(\Sigma)$.

The action of $\operatorname{Diff}^{+}(\Sigma)_{\text {res }}$ on $\pi_{1}(\Sigma, x)$ descends to an action of $\Gamma(\Sigma)$ by group automorphisms. This is amounts to

$$
\chi: \Gamma(\Sigma) \longrightarrow \operatorname{Aut}\left(\pi_{1}(\Sigma, x)\right), \quad[\phi] \mapsto \phi_{*}, \quad[\phi] \in \Gamma(\Sigma)
$$

being a well-defined group morphism, where $\phi_{*}: \pi_{1}(\Sigma, x) \longrightarrow \pi_{1}(\Sigma, x)$ is the map of fundamental groups induced by $\phi \in \operatorname{Diff}^{+}(\Sigma)_{\text {res }}$. One then has the following well-known fact (cf. [5, Section 1.1.1]).

Proposition 4.3.4. The group morphism $\chi: \Gamma(\Sigma) \longrightarrow \operatorname{Aut}\left(\pi_{1}(\Sigma, x)\right)$ is injective.

Now let $\gamma_{1}$ and $\gamma_{2}$ be as chosen earlier. The Dehn twist about $\gamma_{1}$ is then the unique element $[\vartheta] \in \Gamma(\Sigma)$ that satisfies

$$
\vartheta_{*}\left(\left[\gamma_{1}\right]\right)=\left[\gamma_{1}\right] \text { and } \vartheta_{*}\left(\left[\gamma_{2}\right]\right)=\left[\gamma_{2}\right]\left[\gamma_{1}\right] .
$$

Let $S: \mathcal{M}(\Sigma) \longrightarrow \mathcal{M}(\Sigma)$ be the diffeomorphism through which $[\vartheta]$ acts on $\mathcal{M}(\Sigma)$, i.e.

$$
S([\nabla])=[\vartheta] \cdot[\nabla], \quad[\nabla] \in \mathcal{M}(\Sigma) .
$$

If we identify $\mathcal{M}(\Sigma)$ with $D(G)$ via $\Phi_{\gamma_{1}, \gamma_{2}}$, then $S$ identifies with a diffeomorphism $R: D(G) \longrightarrow$ $D(G)$. We thereby obtain the commutative diagram

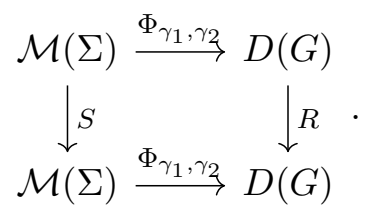

Proposition 4.3.5. The diffeomorphism $R: D(G) \longrightarrow D(G)$ is given by

$$
R(g, h)=\left(g, h g^{-1}\right), \quad(g, h) \in D(G),
$$

and it is a quasi-Hamiltonian G-space automorphism.

Proof. Since $\Gamma(\Sigma)$ acts on $\mathcal{M}(\Sigma)$ by quasi-Hamiltonian $G$-space automorphisms, $S$ is an automorphism of this type. This observation combines with Theorem 4.3.2 and the diagram (17) to imply that $R$ is a quasi-Hamiltonian $G$-space automorphism.

It remains only to verify that $R(g, h)=\left(g, h g^{-1}\right)$ for all $(g, h) \in D(G)$. To this end, fix $(g, h) \in$ $D(G)$ and let $[\nabla] \in \mathcal{M}(\Sigma)$ be such that $\Phi_{\gamma_{1}, \gamma_{2}}([\nabla])=(g, h)$. This amounts to the conditions

$$
\operatorname{Hol}_{\gamma_{1}}(\nabla)=g \text { and } \operatorname{Hol}_{\gamma_{2}}(\nabla)=h .
$$

We have

$$
\operatorname{Hol}_{\gamma_{1}}(\nabla)=\operatorname{Hol}_{\vartheta \circ \gamma_{1}}\left(\left(\vartheta^{-1}\right)^{*} \nabla\right) \text { and } \operatorname{Hol}_{\gamma_{2}}(\nabla)=\operatorname{Hol}_{\vartheta \circ \gamma_{2}}\left(\left(\vartheta^{-1}\right)^{*} \nabla\right) .
$$

On the other hand, the relations (16) imply that

$$
\operatorname{Hol}_{\vartheta \circ \gamma_{1}}\left(\left(\vartheta^{-1}\right)^{*} \nabla\right)=\operatorname{Hol}_{\gamma_{1}}\left(\left(\vartheta^{-1}\right)^{*} \nabla\right) \quad \text { and } \quad \operatorname{Hol}_{\vartheta \circ \gamma_{2}}\left(\left(\vartheta^{-1}\right)^{*} \nabla\right)=\operatorname{Hol}_{\gamma_{2}}\left(\left(\vartheta^{-1}\right)^{*} \nabla\right) \operatorname{Hol}_{\gamma_{1}}\left(\left(\vartheta^{-1}\right)^{*} \nabla\right) \text {. }
$$


These combine with (18) and (19) to imply that

$$
\operatorname{Hol}_{\gamma_{1}}\left(\left(\vartheta^{-1}\right)^{*} \nabla\right)=g \quad \text { and } \operatorname{Hol}_{\gamma_{2}}\left(\left(\vartheta^{-1}\right)^{*} \nabla\right)=h g^{-1} .
$$

Using this and the commutativity of (17), we obtain

$$
\begin{aligned}
R(g, h) & =R\left(\Phi_{\gamma_{1}, \gamma_{2}}([\nabla])\right) \\
& =\Phi_{\gamma_{1}, \gamma_{2}}(S([\nabla])) \\
& =\Phi_{\gamma_{1}, \gamma_{2}}\left(\left[\left(\vartheta^{-1}\right)^{*} \nabla\right]\right) \\
& =\left(\operatorname{Hol}_{\gamma_{1}}\left(\left(\vartheta^{-1}\right)^{*} \nabla\right), \operatorname{Hol}_{\gamma_{2}}\left(\left(\vartheta^{-1}\right)^{*} \nabla\right)\right) \\
& =\left(g, h g^{-1}\right)
\end{aligned}
$$

4.4. The symplectic submanifold $T \times T$. Recall our fixed maximal torus $T \subseteq G$ and its Lie algebra $\mathfrak{t} \subseteq \mathfrak{g}$. The manifold $T \times T$ then carries a symplectic form $\omega_{T \times T} \in \Omega^{2}(T \times T)$, defined as follows on each tangent space $T_{(g, h)}(T \times T)=T_{g} T \oplus T_{h} T$ :

$$
\left(\omega_{T \times T}\right)_{(g, h)}\left(\left(\left(d L_{g}\right)_{e}\left(\xi_{1}\right),\left(d L_{h}\right)_{e}\left(\xi_{2}\right)\right),\left(\left(d L_{g}\right)_{e}\left(\eta_{1}\right),\left(d L_{h}\right)_{e}\left(\eta_{2}\right)\right)\right)=\left\langle\xi_{1}, \eta_{2}\right\rangle-\left\langle\eta_{1}, \xi_{2}\right\rangle
$$

for all $\xi_{1}, \xi_{2}, \eta_{1}, \eta_{2} \in \mathfrak{t}$. This symplectic form turns out to be compatible with the quasi-Hamiltonian form on $D(G)$.

Proposition 4.4.1. We have $j^{*}\left(\omega_{D(G)}\right)=\omega_{T \times T}$, where $j: T \times T \longrightarrow D(G)$ is the inclusion map.

Proof. Let us fix elements $g, h \in T$ and $\xi_{1}, \xi_{2}, \eta_{1}, \eta_{2} \in \mathfrak{t}$. Our task is to prove that

$$
\left(\omega_{D(G)}\right)_{(g, h)}\left(\left(\left(d L_{g}\right)_{e}\left(\xi_{1}\right),\left(d L_{h}\right)_{e}\left(\xi_{2}\right)\right),\left(\left(d L_{g}\right)_{e}\left(\eta_{1}\right),\left(d L_{h}\right)_{e}\left(\eta_{2}\right)\right)\right)=\left\langle\xi_{1}, \eta_{2}\right\rangle-\left\langle\eta_{1}, \xi_{2}\right\rangle .
$$

To this end, consider the two-forms on $D(G)$ defined by

$$
\alpha:=\frac{1}{2}\left(\pi_{1}^{*} \theta^{L}, \pi_{2}^{*} \theta^{R}\right), \quad \beta:=\frac{1}{2}\left(\pi_{1}^{*} \theta^{R}, \pi_{2}^{*} \theta^{L}\right), \quad \text { and } \quad \gamma:=\frac{1}{2}\left(\left(\pi_{1} \pi_{2}\right)^{*} \theta^{L},\left(\pi_{1}^{-1} \pi_{2}^{-1}\right)^{*} \theta^{R}\right) .
$$

One has $\omega_{D(G)}=\alpha+\beta+\gamma$, and it therefore suffices to prove the following identities:

$$
\begin{aligned}
& \alpha_{(g, h)}\left(\left(\left(d L_{g}\right)_{e}\left(\xi_{1}\right),\left(d L_{h}\right)_{e}\left(\xi_{2}\right)\right),\left(\left(d L_{g}\right)_{e}\left(\eta_{1}\right),\left(d L_{h}\right)_{e}\left(\eta_{2}\right)\right)\right)=\frac{1}{2}\left(\left\langle\xi_{1}, \eta_{2}\right\rangle-\left\langle\eta_{1}, \xi_{2}\right\rangle\right) ; \\
& \beta_{(g, h)}\left(\left(\left(d L_{g}\right)_{e}\left(\xi_{1}\right),\left(d L_{h}\right)_{e}\left(\xi_{2}\right)\right),\left(\left(d L_{g}\right)_{e}\left(\eta_{1}\right),\left(d L_{h}\right)_{e}\left(\eta_{2}\right)\right)\right)=\frac{1}{2}\left(\left\langle\xi_{1}, \eta_{2}\right\rangle-\left\langle\eta_{1}, \xi_{2}\right\rangle\right) ; \\
& \gamma_{(g, h)}\left(\left(\left(d L_{g}\right)_{e}\left(\xi_{1}\right),\left(d L_{h}\right)_{e}\left(\xi_{2}\right)\right),\left(\left(d L_{g}\right)_{e}\left(\eta_{1}\right),\left(d L_{h}\right)_{e}\left(\eta_{2}\right)\right)\right)=0 .
\end{aligned}
$$

We begin by verifying (21a). First note that $T$ being abelian forces $\left(d L_{k}\right)_{e}(x)=\left(d R_{k}\right)_{e}(x)$ to hold for all $k \in T$ and $x \in \mathfrak{t}$. We conclude that

$$
\left(\theta^{L}\right)_{k}\left(\left(d L_{k}\right)_{e}(x)\right)=x=\left(\theta^{R}\right)_{k}\left(\left(d L_{k}\right)_{e}(x)\right)
$$


for all $k \in T$ and $x \in \mathfrak{t}$, and this implies the following identities:

$$
\begin{aligned}
& \left(\theta^{L}\right)_{g}\left(\left(d L_{g}\right)_{e}\left(\xi_{1}\right)\right)=\xi_{1} ; \\
& \left(\theta^{L}\right)_{g}\left(\left(d L_{g}\right)_{e}\left(\eta_{1}\right)\right)=\eta_{1} ; \\
& \left(\theta^{R}\right)_{h}\left(\left(d L_{h}\right)_{e}\left(\xi_{2}\right)\right)=\xi_{2} ; \\
& \left(\theta^{R}\right)_{h}\left(\left(d L_{h}\right)_{e}\left(\eta_{2}\right)\right)=\eta_{2} .
\end{aligned}
$$

It follows that

$$
\begin{aligned}
& \alpha_{(g, h)}\left(\left(\left(d L_{g}\right)_{e}\left(\xi_{1}\right),\left(d L_{h}\right)_{e}\left(\xi_{2}\right)\right),\left(\left(d L_{g}\right)_{e}\left(\eta_{1}\right),\left(d L_{h}\right)_{e}\left(\eta_{2}\right)\right)\right) \\
& =\frac{1}{2}\left(\left\langle\left(\theta^{L}\right)_{g}\left(\left(d L_{g}\right)_{e}\left(\xi_{1}\right)\right),\left(\theta^{R}\right)_{h}\left(\left(d L_{h}\right)_{e}\left(\eta_{2}\right)\right)\right\rangle-\left\langle\left(\theta^{L}\right)_{g}\left(\left(d L_{g}\right)_{e}\left(\eta_{1}\right)\right),\left(\theta^{R}\right)_{h}\left(\left(d L_{h}\right)_{e}\left(\xi_{2}\right)\right)\right\rangle\right) \\
& =\frac{1}{2}\left(\left\langle\xi_{1}, \eta_{2}\right\rangle-\left\langle\eta_{1}, \xi_{2}\right\rangle\right) .
\end{aligned}
$$

The verification of (21b) proceeds analogously.

It remains only to prove (21c). To this end, it is straightforward to verify the following identities:

$$
\begin{aligned}
& d\left(\pi_{1} \pi_{2}\right)_{(g, h)}\left(\left(d L_{g}\right)_{e}\left(\xi_{1}\right),\left(d L_{h}\right)_{e}\left(\xi_{2}\right)\right)=\left(d L_{g h}\right)_{e}\left(\xi_{1}+\xi_{2}\right) ; \\
& d\left(\pi_{1} \pi_{2}\right)_{(g, h)}\left(\left(d L_{g}\right)_{e}\left(\eta_{1}\right),\left(d L_{h}\right)_{e}\left(\eta_{2}\right)\right)=\left(d L_{g h}\right)_{e}\left(\eta_{1}+\eta_{2}\right) ; \\
& d\left(\pi_{1}^{-1} \pi_{2}^{-1}\right)_{(g, h)}\left(\left(d L_{g}\right)_{e}\left(\xi_{1}\right),\left(d L_{h}\right)_{e}\left(\xi_{2}\right)\right)=-\left(d L_{g^{-1} h^{-1}}\right)_{e}\left(\xi_{1}+\xi_{2}\right) ; \\
& d\left(\pi_{1}^{-1} \pi_{2}^{-1}\right)_{(g, h)}\left(\left(d L_{g}\right)_{e}\left(\eta_{1}\right),\left(d L_{h}\right)_{e}\left(\eta_{2}\right)\right)=-\left(d L_{g^{-1} h^{-1}}\right)_{e}\left(\eta_{1}+\eta_{2}\right) .
\end{aligned}
$$

One may also use (22) to deduce the following identities:

$$
\begin{aligned}
& \left(\theta^{L}\right)_{g h}\left(\left(d L_{g h}\right)_{e}\left(\xi_{1}+\xi_{2}\right)\right)=\xi_{1}+\xi_{2} \\
& \left(\theta^{L}\right)_{g h}\left(\left(d L_{g h}\right)_{e}\left(\eta_{1}+\eta_{2}\right)\right)=\eta_{1}+\eta_{2} \\
& \left(\theta^{R}\right)_{g^{-1} h^{-1}}\left(\left(d L_{g^{-1} h^{-1}}\right)_{e}\left(\xi_{1}+\xi_{2}\right)\right)=\xi_{1}+\xi_{2} \\
& \left(\theta^{R}\right)_{g^{-1} h^{-1}}\left(\left(d L_{g^{-1} h^{-1}}\right)_{e}\left(\eta_{1}+\eta_{2}\right)\right)=\eta_{1}+\eta_{2} .
\end{aligned}
$$

These last two sentences imply that

$$
\begin{aligned}
& \gamma_{(g, h)}\left(\left(\left(d L_{g}\right)_{e}\left(\xi_{1}\right),\left(d L_{h}\right)_{e}\left(\xi_{2}\right)\right),\left(\left(d L_{g}\right)_{e}\left(\eta_{1}\right),\left(d L_{h}\right)_{e}\left(\eta_{2}\right)\right)\right) \\
& =-\frac{1}{2}\left\langle\left(\theta^{L}\right)_{g h}\left(\left(d L_{g h}\right)_{e}\left(\xi_{1}+\xi_{2}\right)\right),\left(\theta^{R}\right)_{g^{-1} h^{-1}}\left(\left(d L_{g^{-1} h^{-1}}\right)_{e}\left(\eta_{1}+\eta_{2}\right)\right)\right\rangle \\
& +\frac{1}{2}\left\langle\left(\theta^{L}\right)_{g h}\left(\left(d L_{g h}\right)_{e}\left(\eta_{1}+\eta_{2}\right)\right),\left(\theta^{R}\right)_{g^{-1} h^{-1}}\left(\left(d L_{g^{-1} h^{-1}}\right)_{e}\left(\xi_{1}+\xi_{2}\right)\right)\right\rangle \\
& =-\frac{1}{2}\left\langle\xi_{1}+\xi_{2}, \eta_{1}+\eta_{2}\right\rangle+\frac{1}{2}\left\langle\eta_{1}+\eta_{2}, \xi_{1}+\xi_{2}\right\rangle \\
& =0
\end{aligned}
$$

completing the proof. 
4.5. A special instance of quasi-Hamiltonian reduction. Recall the moment map $\mu_{D(G)}$ : $D(G) \longrightarrow G$ and set $D(G)_{\text {reg }}:=G \times G_{\text {reg. }}$. Let us also define

$$
\mu_{D(G)}^{-1}(e)_{\mathrm{reg}}:=\mu_{D(G)}^{-1}(e) \cap D(G)_{\mathrm{reg}} .
$$

Lemma 4.5.1. If $x \in \mu_{D(G)}^{-1}(e)_{\mathrm{reg}}$, then $G_{x}$ is a maximal torus in $G$.

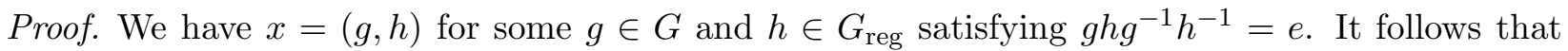
$G_{h}$ is a maximal torus in $G$ containing $g$, and we conclude that $G_{h} \subseteq G_{g}$. Since $G_{x}=G_{g} \cap G_{h}$, we conclude that $G_{x}=G_{h}$. This completes the proof.

This leads to the following additional fact. To this end, recall that $n$ and $r$ denote the dimension and rank of $G$, respectively.

Lemma 4.5.2. The differential of $\mu_{D(G)}$ has constant rank $n-r$ on $\mu_{D(G)}^{-1}(e)_{\mathrm{reg}}$.

Proof. Suppose that $(g, h) \in \mu_{D(G)}^{-1}(e)_{\text {reg }}$ and set $x:=(g, h)$ for notational simplicity. An application of [1, Proposition 4.1] then yields

$$
\operatorname{ker}\left(\left(d \mu_{D(G)}\right)_{x}\right)^{\left(\omega_{D(G)}\right)_{x}}=\left\{\left(\xi_{D(G)}\right)_{x}: \xi \in \mathfrak{g}\right\},
$$

where the the left-hand side denotes the annihilator of $\operatorname{ker}\left(\left(d \mu_{D(G)}\right)_{x}\right) \subseteq T_{x}(D(G))$ under $\left(\omega_{D(G)}\right)_{x}$. The right-hand side has dimension $n-\operatorname{dim}\left(G_{x}\right)$, which by Lemma 4.5.1 coincides with $n-r$. On the other hand, the non-degeneracy of $\left(\omega_{D(G)}\right)_{x}$ (see Remark 4.1.2) forces $\operatorname{ker}\left(\left(d \mu_{D(G)}\right)_{x}\right)^{\left(\omega_{D(G)}\right)_{x}}$ to have dimension $\operatorname{dim}(D(G))-\operatorname{dim}\left(\operatorname{ker}\left(\left(d \mu_{D(G)}\right)_{x}\right)\right.$. It follows that

$$
\operatorname{dim}(D(G))-\operatorname{dim}\left(\operatorname{ker}\left(\left(d \mu_{D(G)}\right)_{x}\right)\right)=n-r
$$

or equivalently

$$
\operatorname{dim}\left(\operatorname{ker}\left(\left(d \mu_{D(G)}\right)_{x}\right)\right)=n+r .
$$

This amounts to $\left(d \mu_{D(G)}\right)_{x}$ having rank $n-r$.

Proposition 4.5.3. The subset $\mu_{D(G)}^{-1}(e)_{\mathrm{reg}} \subseteq D(G)$ is a locally closed, G-invariant submanifold of dimension $n+r$.

Proof. Since $\mu_{D(G)}^{-1}(e)$ and $D(G)_{\text {reg }}$ are $G$-invariant subsets of $D(G)$, the same is true of $\mu_{D(G)}^{-1}(e)_{\text {reg }}=$ $\mu_{D(G)}^{-1}(e) \cap\left(D(G)_{\text {reg }}\right)$. The rest of this proposition follows immediately from Lemma 4.5.2.

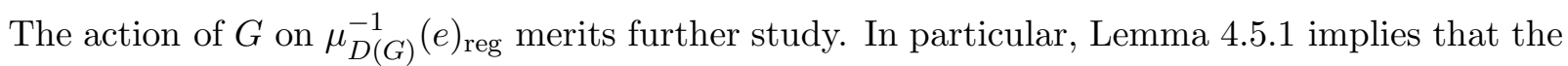
$G$-stabilizers of any two points in $\mu_{D(G)}^{-1}(e)_{\text {reg }}$ are conjugate. The topological quotient $\mu_{D(G)}^{-1}(e)_{\mathrm{reg}} / G$ therefore carries a unique manifold structure for which the quotient map

$$
q: \mu_{D(G)}^{-1}(e)_{\mathrm{reg}} \longrightarrow \mu_{D(G)}^{-1}(e)_{\mathrm{reg}} / G
$$

is a submersion.

Proposition 4.5.4. Let $j: \mu_{D(G)}^{-1}(e)_{\mathrm{reg}} \longrightarrow D(G)$ denote the inclusion map. The quotient manifold $\mu_{D(G)}^{-1}(e)_{\mathrm{reg}} / G$ then carries a unique symplectic form $\overline{\omega_{D(G)}}$ for which $q^{*} \overline{\omega_{D(G)}}=j^{*} \omega_{D(G)}$.

Proof. The arguments given in the proof of [1, Proposition 5.1] apply in our setting.

Remark 4.5.5. The statement of [1, Proposition 5.1] does not apply to the setting of Proposition 4.5.4 it would apply only if the $G$-action on $\mu_{D(G)}^{-1}(e)_{\text {reg }}$ were free. One must examine the proof of [1, Proposition 5.1] to deduce Proposition 4.5.4. 
Now consider the Weyl group $W:=N_{G}(T) / T$ and its action on $T \times T$ defined by

$$
w \cdot(s, t):=\left(w s w^{-1}, w t w^{-1}\right), \quad w \in W,(s, t) \in T \times T .
$$

The subset $T \times T_{\text {reg }} \subseteq T \times T$ is $W$-invariant, and we observe that $W$ acts freely on $T \times T_{\text {reg. }}$. We also observe that the inclusion $T \times T_{\text {reg }} \longrightarrow \mu_{D(G)}^{-1}(e)_{\text {reg }}$ descends to a well-defined smooth map

$$
\varphi:\left(T \times T_{\mathrm{reg}}\right) / W \longrightarrow \mu_{D(G)}^{-1}(e)_{\mathrm{reg}} / G .
$$

At the same time, observe that the symplectic form $\omega_{T \times T} \in \Omega^{2}(T \times T)$ is $W$-invariant. It therefore induces a symplectic form $\overline{\omega_{T \times T}}$ on $\left(T \times T_{\text {reg }}\right) / W$.

Proposition 4.5.6. The map $\varphi:\left(T \times T_{\mathrm{reg}}\right) / W \longrightarrow \mu_{D(G)}^{-1}(e)_{\mathrm{reg}} / G$ is a symplectomorphism from $\left(\left(T \times T_{\mathrm{reg}}\right) / W, \overline{\omega_{T \times T}}\right)$ to $\left(\mu_{D(G)}^{-1}(e)_{\mathrm{reg}} / G, \overline{\omega_{D(G)}}\right)$.

Proof. We begin by verifying that $\varphi^{*}\left(\overline{\omega_{D(G)}}\right)=\overline{\omega_{T \times T}}$. Consider the commutative diagram

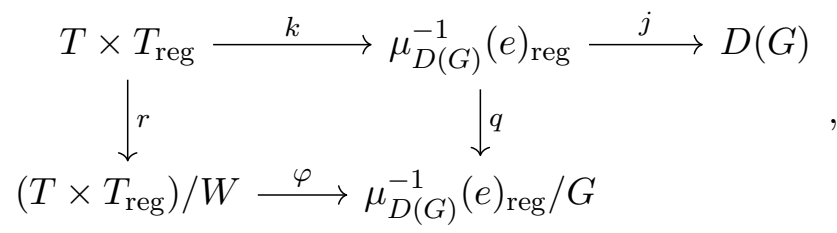

where $j$ and $k$ (resp. $q$ and $r$ ) are the natural inclusions (resp. quotient maps). We have

$$
\begin{aligned}
r^{*}\left(\varphi^{*}\left(\overline{\omega_{D(G)}}\right)\right) & =k^{*}\left(q^{*}\left(\overline{\omega_{D(G)}}\right)\right) \\
& =k^{*}\left(j^{*}\left(\omega_{D(G)}\right)\right) \\
& =\ell^{*}\left(\omega_{D(G)}\right),
\end{aligned}
$$

where $\ell: T \times T_{\text {reg }} \longrightarrow D(G)$ is the inclusion. Proposition 5.5 .2 implies that $\ell^{*}\left(\omega_{D(G)}\right)$ is the restriction of $\omega_{T \times T} \in \Omega^{2}(T \times T)$ to the open subset $T \times T_{\text {reg }} \subseteq T \times T$, and we know this restriction to be $r^{*}\left(\overline{\omega_{T \times T}}\right)$. It follows that $r^{*}\left(\varphi^{*}\left(\overline{\omega_{D(G)}}\right)\right)=r^{*}\left(\overline{\omega_{T \times T}}\right)$, yielding $\varphi^{*}\left(\overline{\omega_{D(G)}}\right)=\overline{\omega_{T \times T}}$.

It remains to verify that $\varphi$ is a diffeomorphism. We first apply Lemma 4.5.1 and Proposition 4.5.3 to conclude that $\mu_{D(G)}^{-1}(e)_{\mathrm{reg}} / G$ is $2 r$-dimensional. The manifold $\left(T \times T_{\mathrm{reg}}\right) / W$ is also $2 r$ dimensional, while the condition $\varphi^{*}\left(\overline{\omega_{D(G)}}\right)=\overline{\omega_{T \times T}}$ forces $\varphi$ to be an immersion. We conclude that $\varphi$ is a local diffeomorphism, and are thereby reduced to proving that $\varphi$ is bijective.

To establish surjectivity, we suppose that $[(g, h)] \in \mu_{D(G)}^{-1}(e)_{\mathrm{reg}} / G$. Note that $k h k^{-1} \in T_{\mathrm{reg}}$ for some $k \in G$, and that $k g k^{-1}$ commutes with $k h k^{-1}$. It follows that $k g k^{-1} \in T$ and

$$
\varphi\left(\left[\left(k g k^{-1}, k h k^{-1}\right)\right]\right)=[(g, h)],
$$

proving surjectivity.

To verify injectivity, assume that $(s, t),\left(s^{\prime}, t^{\prime}\right) \in T \times T_{\text {reg }}$ satisfy $[(s, t)]=\left[\left(s^{\prime}, t^{\prime}\right)\right]$ in $\mu_{D(G)}^{-1}(e)_{\mathrm{reg}} / G$. This assumption amounts to the existence of $g \in G$ for which $s^{\prime}=g s g^{-1}$ and $t^{\prime}=g t g^{-1}$. We may therefore find $w \in W$ with $t^{\prime}=w t w^{-1}$. Now choose a lift $h \in N_{G}(T)$ of $w$ and note that

$$
g t g^{-1}=t^{\prime}=h t h^{-1} \text {. }
$$

Hence

$$
\left(g^{-1} h\right) t\left(g^{-1} h\right)^{-1}=t,
$$

and this combines with the fact that $t \in T_{\text {reg }}$ to yield $g^{-1} h \in T$. It follows that

$$
s^{\prime}=g s g^{-1}=g\left(\left(g^{-1} h\right) s\left(g^{-1} h\right)^{-1}\right) g^{-1}=h s h^{-1}=w s w^{-1},
$$


so that $\left[\left(s^{\prime}, t^{\prime}\right)\right]=\left[\left(w s w^{-1}, w t w^{-1}\right)\right]=[(s, t)]$ in $\left(T \times T_{\text {reg }}\right) / W$. This shows $\varphi$ to be injective, completing the proof.

Fix a point $(s, t) \in T \times T_{\text {reg }}$ and set $x:=(s, t)$. Write $\bar{x}$ and $[x]$ for the equivalence classes of $x$ in $\left(T \times T_{\text {reg }}\right) / W$ and $\mu_{D(G)}^{-1}(e)_{\mathrm{reg}} / G$, respectively. The differential of $\varphi$ at $\bar{x}$ is then a symplectic vector space isomorphism

$$
(d \varphi)_{\bar{x}}: T_{\bar{x}}\left(\left(T \times T_{\mathrm{reg}}\right) / W\right) \stackrel{\cong}{\longrightarrow} T_{[x]}\left(\mu_{D(G)}^{-1}(e)_{\mathrm{reg}} / G\right)
$$

with respect to the forms $\left(\overline{\omega_{T \times T}}\right)_{\bar{x}}$ and $\left(\overline{\omega_{D(G)}}\right)_{[x]}$. At the same time, we have

$$
T_{\bar{x}}\left(\left(T \times T_{\mathrm{reg}}\right) / W\right)=T_{x}\left(T \times T_{\mathrm{reg}}\right)=T_{x}(T \times T) \quad \text { and } \quad T_{[x]}\left(\mu_{D(G)}^{-1}(e)_{\mathrm{reg}} / G\right)=\frac{T_{x}\left(\mu_{D(G)}^{-1}(e)_{\mathrm{reg}}\right)}{T_{x}(G x)} .
$$

Our isomorphism $(d \varphi)_{\bar{x}}$ then takes the form

$$
(d \varphi)_{\bar{x}}: T_{x}(T \times T) \longrightarrow \frac{T_{x}\left(\mu_{D(G)}^{-1}(e)_{\mathrm{reg}}\right)}{T_{x}(G x)}, \quad v \mapsto[v], \quad v \in T_{x}(T \times T),
$$

where $[v] \in \frac{T_{x}\left(\mu_{D(G)}^{-1}(e)_{\mathrm{reg}}\right)}{T_{x}(G x)}$ denotes the equivalence class of $v \in T_{x}(T \times T) \subseteq T_{x}\left(\mu_{D(G)}^{-1}(e)_{\mathrm{reg}}\right)$. We also note that $\left(\overline{\omega_{T \times T}}\right)_{\bar{x}}=\left(\omega_{T \times T}\right)_{x}$ as bilinear forms on $T_{\bar{x}}\left(\left(T \times T_{\text {reg }}\right) / W\right)=T_{x}(T \times T)$. The condition that $(d \varphi)_{\bar{x}}$ respects symplectic forms then becomes the statement

$$
\left(\overline{\omega_{D(G)}}\right)_{[x]}([v],[w])=\left(\omega_{T \times T}\right)_{x}(v, w)
$$

for all $v, w \in T_{x}(T \times T)$.

4.6. Universal centralizers of conjugacy classes. Recall the Lagrangian submanifolds $\Lambda_{\mathcal{O}} \subseteq$ $T^{*} G$ discussed in Section 3. The quasi-Hamiltonian counterparts of the $\Lambda_{\mathcal{O}}$ are the

$$
\Lambda_{\mathcal{C}}:=\left\{(g, h) \in D(G): h \in \mathcal{C} \text { and } g h g^{-1}=h\right\},
$$

where $\mathcal{C} \subseteq G$ ranges over the regular conjugacy classes. Note that

$$
\Lambda_{\mathcal{C}} \longrightarrow \mathcal{C}, \quad(g, h) \mapsto h, \quad(g, h) \in \Lambda_{\mathcal{C}}
$$

defines a fibre bundle in which the fibre over each $h \in \mathcal{C}$ is the maximal torus $G_{h}$. In light of this, we call $\Lambda_{\mathcal{C}}$ the universal centralizer of $\mathcal{C}$. This object exemplifies Definition 4.1.3, as our next result demonstrates.

Theorem 4.6.1. If $\mathcal{C} \subseteq G$ is a regular conjugacy class, then the universal centralizer $\Lambda_{\mathcal{C}}$ is a $G$-invariant, quasi-Hamiltonian Lagrangian submanifold of $D(G)$.

Proof. Our $G$-invariance claim follows from a direct calculation. To establish the rest of our proposition, we recall the requirements of Definition 4.1.3. This leads us to observe that $\Lambda_{\mathcal{C}} \subseteq \mu_{D(G)}^{-1}(e) \subseteq$ $(D(G))^{\circ}$. We also recall that $\Lambda_{\mathcal{C}}$ is a fibre bundle over $\mathcal{C}$, and that the fibres are maximal tori of $G$. It follows that

$$
\operatorname{dim}\left(\Lambda_{\mathcal{C}}\right)=\operatorname{dim}(\mathcal{C})+r=(n-r)+r=n=\frac{1}{2} \operatorname{dim}(D(G)) .
$$

These considerations reduce us to proving that $\Lambda_{\mathcal{C}}$ is isotropic in $D(G)$.

Consider the commutative diagram

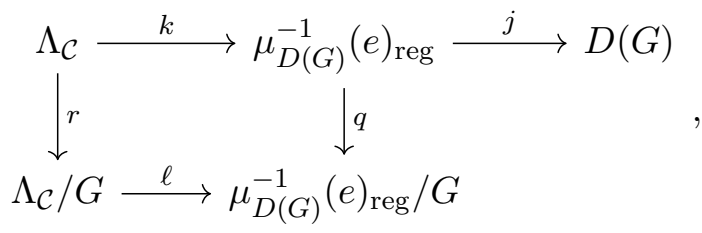


where $j, k$, and $\ell$ (resp. $q$ and $r$ ) are the natural inclusions (resp. quotient maps). Observe that $k^{*}\left(j^{*} \omega_{D(G)}\right)$ is the pullback of $\omega_{D(G)}$ to $\Lambda_{\mathcal{C}}$. On the other hand, Proposition 4.5.4 and the commutitivity of our diagram imply that

$$
k^{*}\left(j^{*} \omega_{D(G)}\right)=k^{*}\left(q^{*} \overline{\omega_{D(G)}}\right)=r^{*}\left(\ell^{*} \overline{\omega_{D(G)}}\right) .
$$

It therefore suffices to prove that $\Lambda_{\mathcal{C}} / G$ is isotropic in the symplectic manifold $\mu_{D(G)}^{-1}(e)_{\mathrm{reg}} / G$.

Recall the symplectomorphism $\varphi:\left(T \times T_{\text {reg }}\right) / W \longrightarrow \mu_{D(G)}^{-1}(e)_{\text {reg }} / G$ discussed in Proposition 4.5.6. Since we have

$$
\varphi((T \times(T \cap \mathcal{C})) / W)=\Lambda_{\mathcal{C}} / G
$$

it suffices to prove that $(T \times(T \cap \mathcal{C})) / W$ is isotropic in $\left(T \times T_{\text {reg }}\right) / W$. This is equivalent to $T \times(T \cap \mathcal{C})$ being isotropic in $T \times T$, which follows from the formula (20) and the fact that $T \cap \mathcal{C}$ is finite. The proof is complete.

\section{BKS PAIRINGS ON THE INTERNALLY FUSED DOUBLE}

Recall the diffeomorphism $R: D(G) \longrightarrow D(G)$ discussed in Section 4.3, We now examine the geometry of $R\left(\Lambda_{\mathcal{C}}\right) \cap \Lambda_{\mathcal{C}^{\prime}}$, where $\mathcal{C}, \mathcal{C}^{\prime} \subseteq G$ are regular conjugacy classes. This will give context for our BKS pairing computation, as described in the introduction of this paper.

5.1. The geometry of $R\left(\Lambda_{\mathcal{C}}\right) \cap \Lambda_{\mathcal{C}^{\prime}}$. Recall the fundamental Weyl alcove $\mathcal{A} \subseteq \mathfrak{t}$ and the associated notation discussed in Section 3.1. Let $\mathcal{C}, \mathcal{C}^{\prime} \subseteq G$ be regular conjugacy classes and set

$$
\beta:=\beta(\mathcal{C}) \in \mathfrak{A} \quad \text { and } \quad \beta^{\prime}:=\beta\left(\mathcal{C}^{\prime}\right) \in \mathfrak{A} .
$$

Let us also recall that $\Lambda_{\mathcal{C}}$ and $\Lambda_{\mathcal{C}^{\prime}}$ are $G$-invariant submanifolds of $D(G)$, and that the diffeomorphism $R: D(G) \longrightarrow D(G)$ is $G$-equivariant. These considerations force the intersection $R\left(\Lambda_{\mathcal{C}}\right) \cap \Lambda_{\mathcal{C}^{\prime}}$ to be $G$-invariant, i.e. $R\left(\Lambda_{\mathcal{C}}\right) \cap \Lambda_{\mathcal{C}^{\prime}}$ is a union of $G$-orbits. To find these $G$-orbits, we first observe that each $w \in W$ determines a point

$$
z_{w}:=\left(\exp \left(w \beta-\beta^{\prime}\right), \exp \left(\beta^{\prime}\right)\right) \in R\left(\Lambda_{\mathcal{C}}\right) \cap \Lambda_{\mathcal{C}^{\prime}}
$$

It follows that

$$
\Gamma_{w}:=G z_{w}
$$

is a $G$-orbit in $R\left(\Lambda_{\mathcal{C}}\right) \cap \Lambda_{\mathcal{C}^{\prime}}$ for all $w \in W$.

Proposition 5.1.1. The association $w \mapsto \Gamma_{w}$ is a bijection from $W$ to the set of $G$-orbits in $R\left(\Lambda_{\mathcal{C}}\right) \cap \Lambda_{\mathcal{C}^{\prime}}$

Proof. We begin with a proof of injectivity, letting $u, w \in W$ be such that $\Gamma_{u}=\Gamma_{w}$. This amounts to the existence of $g \in G$ for which $g \cdot z_{u}=z_{w}$, i.e.

$$
\left(g \exp \left(u \beta-\beta^{\prime}\right) g^{-1}, g \exp \left(\beta^{\prime}\right) g^{-1}\right)=\left(\exp \left(w \beta-\beta^{\prime}\right), \exp \left(\beta^{\prime}\right)\right) .
$$

Since $\beta^{\prime}$ is a regular element of $\mathfrak{t}$, the condition $g \exp \left(\beta^{\prime}\right) g^{-1}=\exp \left(\beta^{\prime}\right)$ implies that $g \in T$. It follows that $\exp \left(u \beta-\beta^{\prime}\right)=\exp \left(w \beta-\beta^{\prime}\right)$, or equivalently that $\exp (u \beta)=\exp (w \beta)$. The regularity of $\beta$ then forces $u=w$ to hold, proving injectivity.

Our claim of surjectivity is equivalent to the following assertion: each point in $R\left(\Lambda_{\mathcal{C}}\right) \cap \Lambda_{\mathcal{C}^{\prime}}$ lies in the $G$-orbit of $z_{w}$ for a suitable $w \in W$. To verify this assertion, we suppose that $(g, h) \in$ $R\left(\Lambda_{\mathcal{C}}\right) \cap \Lambda_{\mathcal{C}^{\prime}}$. It follows that $h \in \mathcal{C}^{\prime}$, i.e. $(g, h)$ lies in the $G$-orbit of a point having $\exp \left(\beta^{\prime}\right)$ as its second coordinate. We may therefore assume that $h=\exp \left(\beta^{\prime}\right)$. Since $g$ commutes with $h=\exp \left(\beta^{\prime}\right)$, we must have $g \in T$. We also have $\left(g, \exp \left(\beta^{\prime}\right)\right) \in R\left(\Lambda_{\mathcal{C}}\right)$, forcing $\exp \left(\beta^{\prime}\right) g \in \mathcal{C}$ to hold. These last two sentences imply that $\exp \left(\beta^{\prime}\right) g \in T \cap \mathcal{C}$, while one knows that $T \cap \mathcal{C}=\{\exp (w \beta): w \in W\}$. Hence $g=\exp \left(w \beta-\beta^{\prime}\right)$ for some $w \in W$, and we see that $(g, h)=z_{w}$. This completes the proof. 
Corollary 5.1.2. The intersection $R\left(\Lambda_{\mathcal{C}}\right) \cap \Lambda_{\mathcal{C}^{\prime}}$ is an $(n-r)$-dimensional submanifold of $D(G)$. Its connected components coincide with its $G$-orbits.

Proof. Note that $T$ is the $G$-stabilizer of $z_{w}$ for each $w \in W$. It follows that $\Gamma_{w}$ has dimension $n-r$ for all $w \in W$. This combines with Proposition 5.1.1 to imply the desired results.

Observe that $R\left(\Lambda_{\mathcal{C}}\right) \subseteq \mu_{D(G)}^{-1}(e)$ and $\Lambda_{\mathcal{C}^{\prime}} \subseteq \mu_{D(G)}^{-1}(e)_{\text {reg }}$, implying that $R\left(\Lambda_{\mathcal{C}}\right) \cap \Lambda_{\mathcal{C}^{\prime}} \subseteq \mu_{D(G)}^{-1}(e)_{\text {reg }}$. In what follows, we fix $x \in R\left(\Lambda_{\mathcal{C}}\right) \cap \Lambda_{\mathcal{C}^{\prime}}$ and consider the three subspaces $T_{x} R\left(\Lambda_{\mathcal{C}}\right), T_{x} \Lambda_{\mathcal{C}^{\prime}}$, and $T_{x}\left(\mu_{D(G)}^{-1}(e)_{\mathrm{reg}}\right)$ of $T_{x}(D(G))$.

Lemma 5.1.3. Suppose that $x \in R\left(\Lambda_{\mathcal{C}}\right) \cap \Lambda_{\mathcal{C}^{\prime}}$ and let $G x \subseteq R\left(\Lambda_{\mathcal{C}}\right) \cap \Lambda_{\mathcal{C}^{\prime}}$ denote the $G$-orbit through $x$. The following statements hold:

(i) $T_{x} R\left(\Lambda_{\mathcal{C}}\right) \cap T_{x} \Lambda_{\mathcal{C}^{\prime}}=T_{x}\left(R\left(\Lambda_{\mathcal{C}}\right) \cap \Lambda_{\mathcal{C}^{\prime}}\right)=T_{x}(G x)$;

(ii) $T_{x} R\left(\Lambda_{\mathcal{C}}\right)+T_{x} \Lambda_{\mathcal{C}^{\prime}}=T_{x}\left(\mu_{D(G)}^{-1}(e)_{\mathrm{reg}}\right)$.

Proof. Corollary 5.1 .2 implies that

$$
T_{x}\left(R\left(\Lambda_{\mathcal{C}}\right) \cap \Lambda_{\mathcal{C}^{\prime}}\right)=T_{x}(G x),
$$

one of the equalities in (i). Using this equality together with the inclusion $T_{x}\left(R\left(\Lambda_{\mathcal{C}}\right) \cap \Lambda_{\mathcal{C}^{\prime}}\right) \subseteq$ $T_{x} R\left(\Lambda_{\mathcal{C}}\right) \cap T_{x} \Lambda_{\mathcal{C}^{\prime}}$ and Corollary [5.1.2, we obtain

$$
\begin{aligned}
\operatorname{dim}\left(T_{x} R\left(\Lambda_{\mathcal{C}}\right)+T_{x} \Lambda_{\mathcal{C}^{\prime}}\right) & =\operatorname{dim}\left(T_{x} R\left(\Lambda_{\mathcal{C}}\right)\right)+\operatorname{dim}\left(T_{x} \Lambda_{\mathcal{C}^{\prime}}\right)-\operatorname{dim}\left(T_{x} R\left(\Lambda_{\mathcal{C}}\right) \cap T_{x} \Lambda_{\mathcal{C}^{\prime}}\right) \\
& \geq \operatorname{dim}\left(T_{x} R\left(\Lambda_{\mathcal{C}}\right)+\operatorname{dim}\left(T_{x} \Lambda_{\mathcal{C}^{\prime}}\right)-\operatorname{dim}\left(T_{x}\left(R\left(\Lambda_{\mathcal{C}}\right) \cap \Lambda_{\mathcal{C}^{\prime}}\right)\right)\right. \\
& =n+n-\operatorname{dim}(G x) \\
& =2 n-(n-r) \\
& =n+r .
\end{aligned}
$$

We also note that the inclusions $R\left(\Lambda_{\mathcal{C}}\right) \subseteq \mu_{D(G)}^{-1}(e)$ and $\Lambda_{\mathcal{C}^{\prime}} \subseteq \mu_{D(G)}^{-1}(e)$ force $T_{x} R\left(\Lambda_{\mathcal{C}}\right)+T_{x} \Lambda_{\mathcal{C}^{\prime}} \subseteq$ $T_{x}\left(\mu_{D(G)}^{-1}(e)_{\text {reg }}\right)$ to hold. Applying Proposition 4.5.3, one obtains

$$
\operatorname{dim}\left(T_{x} R\left(\Lambda_{\mathcal{C}}\right)+T_{x} \Lambda_{\mathcal{C}^{\prime}}\right) \leq n+r .
$$

It follows that (28) and (32) are equalities. Lines (27)-(32) now imply that

$$
\operatorname{dim}\left(T_{x} R\left(\Lambda_{\mathcal{C}}\right) \cap T_{x} \Lambda_{\mathcal{C}^{\prime}}\right)=\operatorname{dim}\left(T_{x}\left(R\left(\Lambda_{\mathcal{C}}\right) \cap \Lambda_{\mathcal{C}^{\prime}}\right)\right),
$$

or equivalently

$$
T_{x}\left(R\left(\Lambda_{\mathcal{C}}\right) \cap \Lambda_{\mathcal{C}^{\prime}}\right)=T_{x} R\left(\Lambda_{\mathcal{C}}\right) \cap T_{x} \Lambda_{\mathcal{C}^{\prime}}
$$

This completes our proof of (i). On the other hand, the equality (32), the inclusion $T_{x} R\left(\Lambda_{\mathcal{C}}\right)+$ $T_{x} \Lambda_{\mathcal{C}^{\prime}} \subseteq T_{x}\left(\mu_{D(G)}^{-1}(e)_{\text {reg }}\right)$, Proposition 4.5.3, and a dimension count yield

$$
\operatorname{dim}\left(T_{x} R\left(\Lambda_{\mathcal{C}}\right)+T_{x} \Lambda_{\mathcal{C}^{\prime}}\right)=\operatorname{dim}\left(T_{x}\left(\mu_{D(G)}^{-1}(e)_{\mathrm{reg}}\right)\right) .
$$

This proves (ii).

5.2. Half-densities on $R\left(\Lambda_{\mathcal{C}}\right)$ and $\Lambda_{\mathcal{C}^{\prime}}$. Recall the brief discussion of conjugacy classes in Section 3.1. Let us now fix a positive integer $k$ and consider a regular, $\frac{1}{k}$-integral conjugacy class $\mathcal{C} \subseteq G$. Let us also set

$$
\beta:=\beta(\mathcal{C}) \in \mathfrak{A}
$$

and write $\mathcal{O} \subseteq \mathfrak{g}$ for the adjoint orbit of $k \beta$. One then has a $G$-equivariant diffeomorphism

$$
\varphi_{\mathcal{O}}^{\mathcal{C}}: \Lambda_{\mathcal{O}} \longrightarrow \Lambda_{\mathcal{C}}, \quad(g, \xi) \mapsto\left(g, \exp \left(\frac{1}{k} \xi\right)\right), \quad(g, \xi) \in \Lambda_{\mathcal{O}}
$$


Lemma 5.2.1. The following assertions are true.

(i) Suppose that $(g, h) \in \Lambda_{\mathcal{C}}$, and let $\xi \in \mathcal{O}$ be the unique element for which $(g, h)=\varphi_{\mathcal{O}}^{\mathcal{C}}(g, \xi)$. The diffeomorphism $\varphi_{\mathcal{O}}^{\mathcal{C}}$ then satisfies

$$
\varphi_{\mathcal{O}}^{\mathcal{C}}(G(g, \xi))=G(g, h) \quad \text { and } \quad \varphi_{\mathcal{O}}^{\mathcal{C}}\left(G_{\xi} \times\{\xi\}\right)=G_{h} \times\{h\} .
$$

(ii) We have the direct sum decomposition

$$
T_{(g, h)} \Lambda_{\mathcal{C}}=T_{(g, h)}(G(g, h)) \oplus T_{(g, h)}\left(G_{h} \times\{h\}\right)
$$

for all $(g, h) \in \Lambda_{\mathcal{C}}$. The $G$-action on $\Lambda_{\mathcal{C}}$ respects these tangent space decompositions.

Proof. Since $\varphi_{\mathcal{O}}^{\mathcal{C}}$ is $G$-equivariant and satisfies $(g, h)=\varphi_{\mathcal{O}}^{\mathcal{C}}(g, \xi)$, we must have $\varphi_{\mathcal{O}}^{\mathcal{C}}(G(g, \xi))=$ $G(g, h)$. The second identity $\varphi_{\mathcal{O}}^{\mathcal{C}}\left(G_{\xi} \times\{\xi\}\right)=G_{h} \times\{h\}$ follows from the definition of $\varphi_{\mathcal{O}}^{\mathcal{C}}$ and the observation that

$$
G_{\xi}=G_{\frac{1}{k} \xi}=G_{\exp \left(\frac{1}{k} \xi\right)}=G_{h} .
$$

This proves (i). The assertion (ii) is a consequence of (i) and Lemma 3.5.1.

Now recall the half-density $\rho_{\mathcal{O}}$ on $\Lambda_{\mathcal{O}}$ constructed in Section 3.5, Let $\rho_{\mathcal{C}}$ denote the half-density on $\Lambda_{\mathcal{C}}$ corresponding to $\rho_{\mathcal{O}}$ under the diffeomorphism (33). This new half-density admits the following description. To prepare for it, we invite the reader to recall the notation and discussion in Sections 3.2 and 3.3 .

Proposition 5.2.2. Fix $(g, h) \in \Lambda_{\mathcal{C}}$ and let $\xi \in \mathcal{O}$ be the unique element satisfying $\exp \left(\frac{1}{k} \xi\right)=h$. Assume that $\left\{\eta_{1}, \ldots, \eta_{n-r}\right\}$ and $\left\{\zeta_{1}, \ldots, \zeta_{r}\right\}$ are bases of $\mathfrak{g}_{\xi}^{\perp}$ and $\mathfrak{g}_{\xi}$, respectively. Write $\widetilde{\eta}_{j}$ for the fundamental vector field on $D(G)$ associated to $\eta_{j}$, i.e.

$$
\widetilde{\eta}_{j}:=\left(\eta_{j}\right)_{D(G)}
$$

for all $j \in\{1, \ldots, n-r\}$. Let us also define the vector

$$
\left(\dot{\zeta}_{k}\right)_{(g, h)}:=\left(\left(d L_{g}\right)_{e}\left(\zeta_{k}\right), 0\right) \in T_{g} G_{\xi} \oplus\{0\}=T_{(g, \xi)}\left(G_{h} \times\{h\}\right) \subseteq T_{(g, h)} \Lambda_{\mathcal{C}}
$$

for each $k \in\{1, \ldots, r\}$. The following statements then hold.

(i) The sets

$$
\left\{\left(\widetilde{\eta_{1}}\right)_{(g, h)}, \ldots,\left(\widetilde{\eta_{n-r}}\right)_{(g, h)}\right\} \quad \text { and } \quad\left\{\left(\dot{\zeta_{1}}\right)_{(g, h)}, \ldots,\left(\dot{\zeta_{r}}\right)_{(g, h)}\right\}
$$

are bases of $T_{(g, h)}(G(g, h))$ and $T_{(g, h)}\left(G_{h} \times\{h\}\right)$, respectively.

(ii) The set

$$
\left\{\left(\widetilde{\eta_{1}}\right)_{(g, h)}, \ldots,\left(\widetilde{\eta_{n-r}}\right)_{(g, h)},\left(\dot{\zeta_{1}}\right)_{(g, h)}, \ldots,\left(\dot{\zeta_{r}}\right)_{(g, h)}\right\}
$$

is a basis of $T_{(g, h)} \Lambda_{\mathcal{C}}$.

(iii) The value of $\left(\rho_{\mathcal{C}}\right)_{(g, h)}$ on this basis is

$$
\kappa(G)^{\frac{1}{2}} \cdot\left|\Omega_{\xi}\left(\eta_{1}, \ldots, \eta_{n-r}\right)\right|^{\frac{1}{2}} .
$$

Proof. Recall the notation in Proposition 3.5.2 and consider the differential of $\varphi_{\mathcal{O}}^{\mathcal{C}}$ at $(g, \xi) \in \Lambda_{\mathcal{O}}$, i.e.

$$
\left(d \varphi_{\mathcal{O}}^{\mathcal{C}}\right)_{(g, \xi)}: T_{(g, \xi)} \Lambda_{\mathcal{O}} \stackrel{\cong}{\longrightarrow} T_{(g, h)} \Lambda_{\mathcal{C}}
$$

Since $\varphi_{\mathcal{O}}^{\mathcal{C}}$ is $G$-equivariant, we must have

$$
\left(d \varphi_{\mathcal{O}}^{\mathcal{C}}\right)_{(g, \xi)}\left(\left(\overline{\eta_{j}}\right)_{(g, \xi)}\right)=\left(\widetilde{\eta}_{j}\right)_{(g, h)}
$$

for all $j \in\{1, \ldots, n-r\}$. It is also straightforward to verify that

$$
\left(d \varphi_{\mathcal{O}}^{\mathcal{C}}\right)_{(g, \xi)}\left(\left(\widehat{\zeta_{k}}\right)_{(g, \xi)}\right)=\left(\dot{\zeta_{k}}\right)_{(g, h)}
$$

for all $k \in\{1, \ldots, r\}$. The desired results now follow from Proposition 3.5 .2 and Lemma 5.2 .1 
Corollary 5.2.3. We have

$$
T_{(g, h)} R\left(\Lambda_{\mathcal{C}}\right)=T_{(g, h)}(G(g, h)) \oplus T_{(g, h)}\left(R\left(G_{h g} \times\{h g\}\right)\right)
$$

for all $(g, h) \in R\left(\Lambda_{\mathcal{C}}\right)$. The $G$-action on $R\left(\Lambda_{\mathcal{C}}\right)$ respects these tangent space decompositions.

Proof. Suppose that $(g, h) \in R\left(\Lambda_{\mathcal{C}}\right)$. We then have $R^{-1}(g, h)=(g, h g) \in \Lambda_{\mathcal{C}}$, in which context Lemma 5.2.1(ii) yields

$$
T_{(g, h g)} \Lambda_{\mathcal{C}}=T_{(g, h g)}(G(g, h g)) \oplus T_{(g, h g)}\left(G_{h g} \times\{h g\}\right) .
$$

It follows that

$$
T_{(g, h)} R\left(\Lambda_{\mathcal{C}}\right)=T_{(g, h)}(R(G(g, h g))) \oplus T_{(g, h)}\left(R\left(G_{h g} \times\{h g\}\right)\right) .
$$

On the other hand, the $G$-equivariance of $R$ implies that $R(G(g, h g))=G(g, h)$. Hence

$$
T_{(g, h)} R\left(\Lambda_{\mathcal{C}}\right)=T_{(g, h)}(G(g, h)) \oplus T_{(g, h)}\left(R\left(G_{h g} \times\{h g\}\right)\right),
$$

as desired.

The arguments in the previous paragraph lead to the following observation: the tangent space decompositions (35) are the result of applying $R$ to the decompositions (34). This combines with Lemma 5.2.1(ii) and the fact that $R$ is a $G$-equivariant diffeomorphism to imply that $G$ respects the decompositions (35). The proof is therefore complete.

Lemma 5.2.4. Fix $(g, h) \in D(G)$ and make the identifications

$$
T_{(g, h)}(D(G))=T_{g} G \oplus T_{h} G \quad \text { and } \quad T_{(g, h g)}(D(G))=T_{g} G \oplus T_{h g} G .
$$

We then have

$$
(d R)_{(g, h g)}\left(\left(d L_{g}\right)_{e}(\zeta), 0\right)=\left(\left(d L_{g}\right)_{e}(\zeta),-\left(d L_{h}\right)_{e}\left(\operatorname{Ad}_{g}(\zeta)\right)\right)
$$

for all $\zeta \in \mathfrak{g}$, where

$$
(d R)_{(g, h g)}: T_{g} G \oplus T_{h g} G \longrightarrow T_{g} G \oplus T_{h} G
$$

is the differential of $R$ at $(g, h g)$.

Proof. We have

$$
\begin{aligned}
(d R)_{(g, h g)}\left(\left(d L_{g}\right)_{e}(\zeta), 0\right) & =\left.\frac{d}{d t}\right|_{t=0} R(g \exp (t \zeta), h g) \\
& =\left.\frac{d}{d t}\right|_{t=0}\left(g \exp (t \zeta), h g \exp (t \zeta)^{-1} g^{-1}\right) \\
& =\left.\frac{d}{d t}\right|_{t=0}\left(g \exp (t \zeta), h \exp \left(-t \operatorname{Ad}_{g}(\zeta)\right)\right) \\
& =\left(\left(d L_{g}\right)_{e}(\zeta),-\left(d L_{h}\right)_{e}\left(\operatorname{Ad}_{g}(\zeta)\right)\right) .
\end{aligned}
$$

Now note that $R$ identifies $\rho_{\mathcal{C}}$ with a half-density $\nu_{\mathcal{C}}$ on $R\left(\Lambda_{\mathcal{C}}\right)$. This leads to the following result.

Proposition 5.2.5. Fix $(g, h) \in R\left(\Lambda_{\mathcal{C}}\right)$ and let $\xi \in \mathcal{O}$ be the unique element satisfying $\exp \left(\frac{1}{k} \xi\right)=$ hg. Assume that $\left\{\eta_{1}, \ldots, \eta_{n-r}\right\}$ and $\left\{\zeta_{1}, \ldots, \zeta_{r}\right\}$ are bases of $\mathfrak{g}_{\xi}^{\perp}$ and $\mathfrak{g}_{\xi}$, respectively. Write $\widetilde{\eta}_{j}$ for the fundamental vector field on $D(G)$ associated to $\eta_{j}$, i.e.

$$
\widetilde{\eta}_{j}:=\left(\eta_{j}\right)_{D(G)}
$$

for all $j \in\{1, \ldots, n-r\}$. Let us also define the vector

$$
\left(\zeta_{k}^{\prime}\right)_{(g, h)}:=\left(\left(d L_{g}\right)_{e}\left(\zeta_{k}\right),-\left(d L_{h}\right)_{e}\left(\zeta_{k}\right)\right) \in T_{(g, h)} R\left(G_{h g} \times\{h g\}\right) \subseteq T_{(g, h)} R\left(\Lambda_{\mathcal{C}}\right)
$$

for each $k \in\{1, \ldots, r\}$. The following statements then hold. 
(i) The sets

$$
\left\{\left(\widetilde{\eta_{1}}\right)_{(g, h)}, \ldots,\left(\widetilde{\eta_{n-r}}\right)_{(g, h)}\right\} \quad \text { and } \quad\left\{\left(\zeta_{1}^{\prime}\right)_{(g, h)}, \ldots,\left(\zeta_{r}^{\prime}\right)_{(g, h)}\right\}
$$

are bases of $T_{(g, h)}(G(g, h))$ and $T_{(g, h)} R\left(G_{h g} \times\{h g\}\right)$, respectively.

(ii) The set

$$
\left\{\left(\widetilde{\eta_{1}}\right)_{(g, h)}, \ldots,\left(\widetilde{\eta_{n-r}}\right)_{(g, h)},\left(\zeta_{1}^{\prime}\right)_{(g, h)}, \ldots,\left(\zeta_{r}^{\prime}\right)_{(g, h)}\right\}
$$

is a basis of $T_{(g, h)} R\left(\Lambda_{\mathcal{C}}\right)$.

(iii) The value of $\left(\nu_{\mathcal{C}}\right)_{(g, h)}$ on this basis is

$$
\kappa(G)^{\frac{1}{2}} \cdot\left|\Omega_{\xi}\left(\eta_{1}, \ldots, \eta_{n-r}\right)\right|^{\frac{1}{2}} .
$$

Proof. Recall the notation used in Proposition 5.2.2, Lemma 5.2.4 then yields

$$
(d R)_{(g, h g)}\left(\left(\dot{\zeta}_{k}\right)_{(g, h g)}\right)=\left(\zeta_{k}^{\prime}\right)_{(g, h)}
$$

for all $k \in\{1, \ldots, r\}$. At the same time, the $G$-equivariance of $R$ implies that

$$
(d R)_{(g, h g)}\left(\left(\widetilde{\eta}_{j}\right)_{(g, h g)}\right)=\left(\widetilde{\eta}_{j}\right)_{(g, h)}
$$

for all $j \in\{1, \ldots, n-r\}$. The desired results now follow from Proposition 5.2 .2 and Corollary 5.2 .3

5.3. Phase functions on $R\left(\Lambda_{\mathcal{C}}\right)$ and $\Lambda_{\mathcal{C}^{\prime}}$. Fix a positive integer $k$ and let $\mathcal{C} \subseteq G$ be a regular, $\frac{1}{k}$-integral conjugacy class. Let $\mathcal{O} \subseteq \mathfrak{g}$ be the adjoint orbit of $k \beta(\mathcal{C})$, and recall the $G$-equivariant diffeomorphism $\varphi_{\mathcal{O}}^{\mathcal{C}}: \Lambda_{\mathcal{O}} \stackrel{\cong}{\longrightarrow} \Lambda_{\mathcal{C}}$ defined in (33). Let us also recall the $G$-invariant phase function $\psi_{\mathcal{O}}: \Lambda_{\mathcal{O}} \longrightarrow S^{1}$ considered in Section 3.4. We then have $\psi_{\mathcal{O}}=\left(\varphi_{\mathcal{O}}^{\mathcal{C}}\right)^{*} \psi_{\mathcal{C}}$, where $\psi_{\mathcal{C}}: \Lambda_{\mathcal{C}} \longrightarrow S^{1}$ is the $G$-invariant map defined as follows:

$$
\psi_{\mathcal{C}}(g, h)=e^{2 \pi i\langle\xi, \eta\rangle},
$$

where $\xi \in \mathcal{O}$ is the unique element for which $\exp \left(\frac{1}{k} \xi\right)=h$ and $\eta \in \mathfrak{g}_{\xi}$ satisfies $g=\exp (\eta)$. At the same time, one can pull $\psi_{\mathcal{C}}$ back along the $G$-equivariant diffeomorphism

$$
\left.R^{-1}\right|_{R\left(\Lambda_{\mathcal{C}}\right)}: R\left(\Lambda_{\mathcal{C}}\right) \stackrel{\cong}{\longrightarrow} \Lambda_{\mathcal{C}}
$$

to obtain a $G$-invariant map $\vartheta_{\mathcal{C}}: R\left(\Lambda_{\mathcal{C}}\right) \longrightarrow S^{1}$. It follows that

$$
\vartheta_{\mathcal{C}}(g, h)=\psi_{\mathcal{C}}(g, h g)
$$

for all $(g, h) \in R\left(\Lambda_{\mathcal{C}}\right)$.

5.4. Some technical lemmas. Fix a positive integer $k$ and let $\mathcal{C}, \mathcal{C}^{\prime} \subseteq G$ be regular, $\frac{1}{k}$-integral conjugacy classes. Write $\mathcal{O}, \mathcal{O}^{\prime} \subseteq \mathfrak{g}$ for the adjoint orbits of $k \beta(\mathcal{C}), k \beta\left(\mathcal{C}^{\prime}\right)$, respectively. Section 5.2 uses this information to construct half-densities $\nu_{\mathcal{C}}$ and $\rho_{\mathcal{C}^{\prime}}$ on $R\left(\Lambda_{\mathcal{C}}\right)$ and $\Lambda_{\mathcal{C}^{\prime}}$, respectively. Let us also recall that $R\left(\Lambda_{\mathcal{C}}\right)$ and $\Lambda_{\mathcal{C}^{\prime}}$ are cleanly intersecting Lagrangian submanifolds of $D(G)$ (see Lemma 5.1.3). It now follows from Section $\mathrm{A3}$ that $\nu_{\mathcal{C}}$ and $\rho_{\mathcal{C}^{\prime}}$ determine a density $D\left(\nu_{\mathcal{C}}, \rho_{\mathcal{C}^{\prime}}\right)$ on $R\left(\Lambda_{\mathcal{C}}\right) \cap \Lambda_{\mathcal{C}^{\prime}}$. Note that

$$
D\left(\nu_{\mathcal{C}}, \rho_{\mathcal{C}^{\prime}}\right)_{x}=\Phi_{x}\left(\left(\nu_{\mathcal{C}}\right)_{x} \otimes\left(\rho_{\mathcal{C}^{\prime}}\right)_{x}\right)
$$

for all $x \in R\left(\Lambda_{\mathcal{C}}\right) \cap \Lambda_{\mathcal{C}^{\prime}}$, where

$$
\Phi_{x}:\left.\left|T_{x} R\left(\Lambda_{\mathcal{C}}\right)^{\frac{1}{2}} \otimes\right| T_{x} \Lambda_{\mathcal{C}^{\prime}}\right|^{\frac{1}{2}} \longrightarrow\left|T_{x}\left(R\left(\Lambda_{\mathcal{C}}\right) \cap \Lambda_{\mathcal{C}^{\prime}}\right)\right|
$$

is the map (56).

In what follows, we describe the density $D\left(\nu_{\mathcal{C}}, \rho_{\mathcal{C}^{\prime}}\right)$ on $R\left(\Lambda_{\mathcal{C}}\right) \cap \Lambda_{\mathcal{C}^{\prime}}$.

Lemma 5.4.1. The density $D\left(\nu_{\mathcal{C}}, \rho_{\mathcal{C}^{\prime}}\right)$ is invariant under the action of $G$ on $R\left(\Lambda_{\mathcal{C}}\right) \cap \Lambda_{\mathcal{C}^{\prime}}$. 
Proof. Recall that $\rho_{\mathcal{O}^{\prime}}$ is $G$-invariant (see Proposition 3.5.3), and that $\rho_{\mathcal{C}^{\prime}}$ corresponds to $\rho_{\mathcal{O}^{\prime}}$ under the $G$-equivariant diffeomorphism $\varphi_{\mathcal{O}^{\prime}}^{\mathcal{C}^{\prime}}: \Lambda_{\mathcal{O}^{\prime}} \stackrel{\cong}{\longrightarrow} \Lambda_{\mathcal{C}^{\prime}}$ from (33). These considerations imply that $\rho_{\mathcal{C}^{\prime}}$ is $G$-invariant, and an analogous argument establishes the invariance of $\rho_{\mathcal{C}}$. At the same time, we recall that $\nu_{\mathcal{C}}$ corresponds to $\rho_{\mathcal{C}}$ under the $G$-equivariant diffeomorphism

$$
\left.R\right|_{\Lambda_{\mathcal{C}}}: \Lambda_{\mathcal{C}} \stackrel{\cong}{\longrightarrow} R\left(\Lambda_{\mathcal{C}}\right) .
$$

We conclude that $\nu_{\mathcal{C}}$ is also $G$-invariant, and the desired result now follows from Lemma A6.1.

We also need the following elementary lemma.

Lemma 5.4.2. If $g, h \in G$ are commuting elements for which $h \in G_{\mathrm{reg}}$ and $h g \in G_{\mathrm{reg}}$, then $G_{h g}=G_{h}$.

Proof. Note that $G_{h g}$ is a maximal torus in $G$. We also have $h \in G_{h g}$, owing to the fact that $h$ and $g$ commute. These last two sentences force $G_{h g} \subseteq G_{h}$ to hold. Since $G_{h}$ is also a maximal torus, we obtain $G_{h g}=G_{h}$.

Now fix $(g, h) \in R\left(\Lambda_{\mathcal{C}}\right) \cap \Lambda_{\mathcal{C}^{\prime}}$ and set $x:=(g, h)$. Lemma 5.4 .2 then gives the equality $G_{h}=G_{h g}$ of maximal tori. No generality will be lost in taking this maximal torus to be our fixed maximal torus T. Applications of Lemma 5.2.1 Corollary 5.2.3 then yield

$$
T_{x} R\left(\Lambda_{\mathcal{C}}\right)=T_{x}(G x) \oplus T_{x} R(T \times\{h g\}) \quad \text { and } \quad T_{x} \Lambda_{\mathcal{C}}=T_{x}(G x) \oplus T_{x}(T \times\{h\}) .
$$

By Lemma 5.1.3(i), these statements may be written as

(38) $T_{x} R\left(\Lambda_{\mathcal{C}}\right)=T_{x}\left(R\left(\Lambda_{\mathcal{C}}\right) \cap \Lambda_{\mathcal{C}^{\prime}}\right) \oplus T_{x} R(T \times\{h g\}) \quad$ and $\quad T_{x} \Lambda_{\mathcal{C}}=T_{x}\left(R\left(\Lambda_{\mathcal{C}}\right) \cap \Lambda_{\mathcal{C}^{\prime}}\right) \oplus T_{x}(T \times\{h\})$.

These combine with Corollary A5.2 to imply that

$$
T_{x} R(T \times\{h g\}) \oplus T_{x}(T \times\{h\}) \longrightarrow \frac{T_{x} R\left(\Lambda_{\mathcal{C}}\right)+T_{x} \Lambda_{\mathcal{C}^{\prime}}}{T_{x}\left(R\left(\Lambda_{\mathcal{C}}\right) \cap \Lambda_{\mathcal{C}^{\prime}}\right)}, \quad\left(v_{1}, v_{2}\right) \mapsto\left[v_{1}-v_{2}\right]
$$

is a vector space isomorphism. Let us consider the induced isomorphism

$$
\left|T_{x} R(T \times\{h g\}) \oplus T_{x}(T \times\{h\})\right|^{\frac{1}{2}} \stackrel{\cong}{\longrightarrow}\left|\frac{T_{x} R\left(\Lambda_{\mathcal{C}}\right)+T_{x} \Lambda_{\mathcal{C}^{\prime}}}{T_{x}\left(R\left(\Lambda_{\mathcal{C}}\right) \cap \Lambda_{\mathcal{C}^{\prime}}\right)}\right|^{\frac{1}{2}} .
$$

Lemma 5.4.3. Use the notation of Propositions 5.2.2 and 5.2.5, and let

$$
\rho \in\left|T_{x} R(T \times\{h g\}) \oplus T_{x}(T \times\{h\})\right|^{\frac{1}{2}}
$$

be the unique half-density satisfying

$$
\rho\left(\left(\left(\zeta_{1}^{\prime}\right)_{x}, 0\right), \ldots,\left(\left(\zeta_{r}^{\prime}\right)_{x}, 0\right),\left(0,\left(\dot{\zeta}_{1}\right)_{x}\right), \ldots,\left(0,\left(\dot{\zeta}_{r}\right)_{x}\right)\right)=\kappa(G) .
$$

If $\rho^{\prime}$ is the image of $\rho$ under (40), then

$$
\rho^{\prime}\left(\left[\left(\left(d L_{g}\right)_{e}\left(\zeta_{1}\right), 0\right)\right], \ldots,\left[\left(\left(d L_{g}\right)_{e}\left(\zeta_{r}\right), 0\right)\right],\left[\left(0,\left(d L_{h}\right)_{e}\left(\zeta_{1}\right)\right)\right], \ldots,\left[\left(0,\left(d L_{h}\right)_{e}\left(\zeta_{r}\right)\right)\right]\right)=\kappa(G) .
$$

Proof. The images of

$$
\left(\left(\zeta_{1}^{\prime}\right)_{x}, 0\right), \ldots,\left(\left(\zeta_{r}^{\prime}\right)_{x}, 0\right),\left(0,\left(\dot{\zeta}_{1}\right)_{x}\right), \ldots,\left(0,\left(\dot{\zeta}_{r}\right)_{x}\right)
$$

under (39) are

$$
\left[\left(\zeta_{1}^{\prime}\right)_{x}\right], \ldots,\left[\left(\zeta_{r}^{\prime}\right)_{x}\right],-\left[\left(\dot{\zeta}_{1}\right)_{x}\right], \ldots,-\left[\left(\dot{\zeta}_{r}\right)_{x}\right]
$$


respectively. It now follows from the definition of (40) that

$$
\begin{aligned}
\rho^{\prime}\left(\left[\left(\zeta_{1}^{\prime}\right)_{x}\right], \ldots,\left[\left(\zeta_{r}^{\prime}\right)_{x}\right],-\left[\left(\dot{\zeta}_{1}\right)_{x}\right], \ldots,-\left[\left(\dot{\zeta}_{r}\right)_{x}\right]\right) & =\rho\left(\left(\left(\zeta_{1}^{\prime}\right)_{x}, 0\right), \ldots,\left(\left(\zeta_{r}^{\prime}\right)_{x}, 0\right),\left(0,\left(\dot{\zeta}_{1}\right)_{x}\right), \ldots,\left(0,\left(\dot{\zeta}_{r}\right)_{x}\right)\right) \\
& =\kappa(G) .
\end{aligned}
$$

This is precisely the statement that

$$
\rho^{\prime}\left(\left[\left(\zeta_{1}^{\prime}\right)_{x}\right], \ldots,\left[\left(\zeta_{r}^{\prime}\right)_{x}\right],\left[\left(\dot{\zeta}_{1}\right)_{x}\right], \ldots,\left[\left(\dot{\zeta}_{r}\right)_{x}\right]\right)=\kappa(G) .
$$

Now consider the linear automorphism

$$
\frac{T_{x} R\left(\Lambda_{\mathcal{C}}\right)+T_{x} \Lambda_{\mathcal{C}^{\prime}}}{T_{x}\left(R\left(\Lambda_{\mathcal{C}}\right) \cap \Lambda_{\mathcal{C}^{\prime}}\right)} \longrightarrow \frac{T_{x} R\left(\Lambda_{\mathcal{C}}\right)+T_{x} \Lambda_{\mathcal{C}^{\prime}}}{T_{x}\left(R\left(\Lambda_{\mathcal{C}}\right) \cap \Lambda_{\mathcal{C}^{\prime}}\right)}
$$

that sends the basis

$$
\left\{\left[\left(\zeta_{1}^{\prime}\right)_{x}\right], \ldots,\left[\left(\zeta_{r}^{\prime}\right)_{x}\right],\left[\left(\dot{\zeta}_{1}\right)_{x}\right], \ldots,\left[\left(\dot{\zeta}_{r}\right)_{x}\right]\right\}
$$

to the basis

$$
\left\{\left[\left(\dot{\zeta}_{1}\right)_{x}\right], \ldots,\left[\left(\dot{\zeta}_{r}\right)_{x}\right],-\left[\left(\zeta_{1}^{\prime}\right)_{x}\right]+\left[\left(\dot{\zeta_{1}}\right)_{x}\right], \ldots,-\left[\left(\zeta_{r}^{\prime}\right)_{x}\right]+\left[\left(\dot{\zeta}_{r}\right)_{x}\right]\right\} .
$$

This automorphism has a determinant of absolute value one, so that

$$
\begin{aligned}
& \rho^{\prime}\left(\left[\left(\dot{\zeta}_{1}\right)_{x}\right], \ldots,\left[\left(\dot{\zeta}_{r}\right)_{x}\right],-\left[\left(\zeta_{1}^{\prime}\right)_{x}\right]+\left[\left(\dot{\zeta}_{1}\right)_{x}\right], \ldots,-\left[\left(\zeta_{r}^{\prime}\right)_{x}\right]+\left[\left(\dot{\zeta}_{r}\right)_{x}\right]\right) \\
& =\rho^{\prime}\left(\left[\left(\zeta_{1}^{\prime}\right)_{x}\right], \ldots,\left[\left(\zeta_{r}^{\prime}\right)_{x}\right],\left[\left(\dot{\zeta}_{1}\right)_{x}\right], \ldots,\left[\left(\dot{\zeta}_{r}\right)_{x}\right]\right) \\
& =\kappa(G) .
\end{aligned}
$$

It remains only to observe that

$$
\left[\left(\dot{\zeta}_{k}\right)_{x}\right]=\left[\left(\left(d L_{g}\right)_{e}\left(\zeta_{k}\right), 0\right)\right] \quad \text { and } \quad-\left[\left(\zeta_{k}^{\prime}\right)_{x}\right]+\left[\left(\dot{\zeta}_{k}\right)_{x}\right]=\left[\left(0,\left(d L_{h}\right)_{e}\left(\zeta_{k}\right)\right)\right]
$$

for all $k \in\{1, \ldots, r\}$.

We now scrutinize the codomain of (39) in greater detail. Section A3 explains that this codomain is a symplectic vector space, and that the symplectic form induces an isomorphism

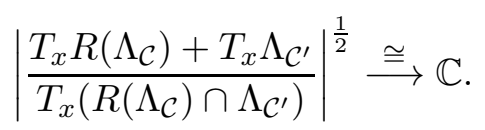

At the same time, Lemma 5.1 .3 implies that

$$
\frac{T_{x} R\left(\Lambda_{\mathcal{C}}\right)+T_{x} \Lambda_{\mathcal{C}^{\prime}}}{T_{x}\left(R\left(\Lambda_{\mathcal{C}}\right) \cap \Lambda_{\mathcal{C}^{\prime}}\right)}=\frac{T_{x}\left(\mu_{D(G)}^{-1}(e)_{\mathrm{reg}}\right)}{T_{x}(G x)}=T_{[x]}\left(\mu_{D(G)}^{-1}(e)_{\mathrm{reg}} / G\right) .
$$

The symplectic form $\left(\overline{\omega_{D(G)}}\right)_{[x]}$ on $T_{[x]}\left(\mu_{D(G)}^{-1}(e)_{\mathrm{reg}} / G\right)$ (see Section 4.5) then agrees with the abovediscussed symplectic form on $\frac{T_{x} R\left(\Lambda_{\mathcal{C}}\right)+T_{x} \Lambda_{\mathcal{C}^{\prime}}}{T_{x}\left(R\left(\Lambda_{\mathcal{C}}\right) \cap \Lambda_{\mathcal{C}^{\prime}}\right)}$, as each is obtained from $\left(\omega_{D(G)}\right)_{x}$ in the same way.

Lemma 5.4.4. Suppose that $\left\{\zeta_{1}, \ldots, \zeta_{r}\right\}$ is an orthonormal basis of $\mathfrak{t}$. If we have

$$
\rho^{\prime} \in\left|\frac{T_{x} R\left(\Lambda_{\mathcal{C}}\right)+T_{x} \Lambda_{\mathcal{C}^{\prime}}}{T_{x}\left(R\left(\Lambda_{\mathcal{C}}\right) \cap \Lambda_{\mathcal{C}^{\prime}}\right)}\right|^{\frac{1}{2}}
$$

then the image of $\rho^{\prime}$ under (41) is equal to

$$
\rho^{\prime}\left(\left[\left(\left(d L_{g}\right)_{e}\left(\zeta_{1}\right), 0\right)\right], \ldots,\left[\left(\left(d L_{g}\right)_{e}\left(\zeta_{r}\right), 0\right)\right],\left[\left(0,\left(d L_{h}\right)_{e}\left(\zeta_{1}\right)\right)\right], \ldots,\left[\left(0,\left(d L_{h}\right)_{e}\left(\zeta_{r}\right)\right)\right]\right) .
$$


Proof. Let $a \in \mathbb{C}$ denote the image of $\rho^{\prime}$ under (41), and recall the paragraph preceding the statement of this lemma. This paragraph implies that

$$
\rho^{\prime}=a\left|\Omega_{[x]}\right|^{\frac{1}{2}},
$$

where $\Omega_{[x]}:=\left(\left(\overline{\omega_{D(G)}}\right)_{[x]}\right)^{r}$ is the highest non-zero wedge power of $\left(\overline{\omega_{D(G)}}\right)_{[x]}$. It therefore suffices to prove that

$$
\left|\Omega_{[x]}\right|\left(\left[\left(\left(d L_{g}\right)_{e}\left(\zeta_{1}\right), 0\right)\right], \ldots,\left[\left(\left(d L_{g}\right)_{e}\left(\zeta_{r}\right), 0\right)\right],\left[\left(0,\left(d L_{h}\right)_{e}\left(\zeta_{1}\right)\right)\right], \ldots,\left[\left(0,\left(d L_{h}\right)_{e}\left(\zeta_{r}\right)\right)\right]\right)=1
$$

Consider the symplectic form $\left(\omega_{T \times T}\right)_{x}$ on $T_{x}(T \times T)$ from Section 4.5, and its highest non-zero wedge power $\Xi_{x}:=\left(\left(\omega_{T \times T}\right)_{x}\right)^{r}$. The relation (26) implies that

$$
\begin{aligned}
& \Omega_{[x]}\left(\left[\left(\left(d L_{g}\right)_{e}\left(\zeta_{1}\right), 0\right)\right], \ldots,\left[\left(\left(d L_{g}\right)_{e}\left(\zeta_{r}\right), 0\right)\right],\left[\left(0,\left(d L_{h}\right)_{e}\left(\zeta_{1}\right)\right)\right], \ldots,\left[\left(0,\left(d L_{h}\right)_{e}\left(\zeta_{r}\right)\right)\right]\right) \\
& =\Xi_{x}\left(\left(\left(d L_{g}\right)_{e}\left(\zeta_{1}\right), 0\right), \ldots,\left(\left(d L_{g}\right)_{e}\left(\zeta_{r}\right), 0\right),\left(0,\left(d L_{h}\right)_{e}\left(\zeta_{1}\right)\right), \ldots,\left(0,\left(d L_{h}\right)_{e}\left(\zeta_{r}\right)\right)\right) .
\end{aligned}
$$

By virtue of the definition (20) and the fact that $\left\{\zeta_{1}, \ldots, \zeta_{r}\right\}$ is orthonormal, the right-hand side has an absolute value of 1 . This completes the proof.

5.5. The main results. Fix a positive integer $k$ and recall the notation in Section 3.1 associated with conjugacy classes. Let $\mathcal{C}, \mathcal{C}^{\prime} \subseteq G$ be regular, $\frac{1}{k}$-integral conjugacy classes and set

$$
\beta:=\beta(\mathcal{C}) \in \mathfrak{A} \quad \text { and } \quad \beta^{\prime}:=\beta\left(\mathcal{C}^{\prime}\right) \in \mathfrak{A} .
$$

One then recalls the definition and properties of $z_{w} \in R\left(\Lambda_{\mathcal{C}}\right) \cap \Lambda_{\mathcal{C}^{\prime}}$ from Section 5.1, where $w \in W$. Let us also recall that $n$ and $r$ denote the dimension and rank of $G$, respectively.

Proposition 5.5.1. Fix $w \in W$, set $x:=z_{w} \in R\left(\Lambda_{\mathcal{C}}\right) \cap \Lambda_{\mathcal{C}^{\prime}}$, and let $\left\{\eta_{1}, \ldots, \eta_{n-r}\right\}$ and be a basis of $\mathfrak{t}^{\perp}$. Let

$$
\widetilde{\eta_{j}}:=\left(\eta_{j}\right)_{D(G)}
$$

be the fundamental vector field on $D(G)$ associated to $\eta_{j}$ for each $j \in\{1, \ldots, n-r\}$. The following statements then hold.

(i) The set $\left\{\left(\widetilde{\eta_{1}}\right)_{x}, \ldots,\left(\widetilde{\eta_{n-r}}\right)_{x}\right\}$ is a basis of $T_{x}\left(R\left(\Lambda_{\mathcal{C}}\right) \cap \Lambda_{\mathcal{C}^{\prime}}\right)$.

(ii) The value of $D\left(\nu_{\mathcal{C}}, \rho_{\mathcal{C}^{\prime}}\right)_{x}$ on this basis is

$$
k^{n-r} \kappa(G) \cdot\left|\Omega_{\beta}\left(\eta_{1}, \ldots, \eta_{n-r}\right)\right|^{\frac{1}{2}}\left|\Omega_{\beta^{\prime}}\left(\eta_{1}, \ldots, \eta_{n-r}\right)\right|^{\frac{1}{2}} .
$$

Proof. Part (i) follows from the identity $T_{x}\left(R\left(\Lambda_{\mathcal{C}}\right) \cap \Lambda_{\mathcal{C}^{\prime}}\right)=T_{x}(G x)$ (see Lemma 5.1.3(i)) and Proposition 5.2.2(i).

We now verify (ii). Note that if we write $z_{w}=(g, h)$, then $h=\exp \left(\beta^{\prime}\right)$ and $h g=\exp (w \beta)$. If $\left\{\zeta_{1}, \ldots, \zeta_{r}\right\}$ is an orthonormal basis of $\mathfrak{t}$, then the previous sentence combines with Propositions 5.2 .2 and 5.2.5 to imply

$$
\left(\rho_{\mathcal{C}^{\prime}}\right)_{x}\left(\left(\widetilde{\eta_{1}}\right)_{x}, \ldots,\left(\widetilde{\eta_{n-r}}\right)_{x},\left(\dot{\zeta_{1}}\right)_{x}, \ldots,\left(\dot{\zeta_{r}}\right)_{x}\right)=\kappa(G)^{\frac{1}{2}} \cdot\left|\Omega_{k \beta^{\prime}}\left(\eta_{1}, \ldots, \eta_{n-r}\right)\right|^{\frac{1}{2}}
$$

and

$$
\left(\nu_{\mathcal{C}}\right)_{x}\left(\left(\widetilde{\eta_{1}}\right)_{x}, \ldots,\left(\widetilde{\eta_{n-r}}\right)_{x},\left(\zeta_{1}^{\prime}\right)_{x}, \ldots,\left(\zeta_{r}^{\prime}\right)_{x}\right)=\kappa(G)^{\frac{1}{2}} \cdot\left|\Omega_{w(k \beta)}\left(\eta_{1}, \ldots, \eta_{n-r}\right)\right|^{\frac{1}{2}} .
$$

Lemma 3.2 .1 tells us that $\left|\Omega_{w(k \beta)}\right|^{\frac{1}{2}}=\left|\Omega_{k \beta}\right|^{\frac{1}{2}}$ as half-densities on $\mathfrak{t}^{\perp}$, allowing us to write

$$
\left(\rho_{\mathcal{C}^{\prime}}\right)_{x}\left(\left(\widetilde{\eta_{1}}\right)_{x}, \ldots,\left(\widetilde{\eta_{n-r}}\right)_{x},\left(\dot{\zeta_{1}}\right)_{x}, \ldots,(\dot{\zeta} r)_{x}\right)=\kappa(G)^{\frac{1}{2}} \cdot\left|\Omega_{k \beta^{\prime}}\left(\eta_{1}, \ldots, \eta_{n-r}\right)\right|^{\frac{1}{2}}
$$


and

$$
\left(\nu_{\mathcal{C}}\right)_{x}\left(\left(\widetilde{\eta_{1}}\right)_{x}, \ldots,\left(\widetilde{\eta_{n-r}}\right)_{x},\left(\zeta_{1}^{\prime}\right)_{x}, \ldots,\left(\zeta_{r}^{\prime}\right)_{x}\right)=\kappa(G)^{\frac{1}{2}} \cdot\left|\Omega_{k \beta}\left(\eta_{1}, \ldots, \eta_{n-r}\right)\right|^{\frac{1}{2}} .
$$

Now recall the tangent space decompositions (38) and set

$$
V_{1}:=T_{x} R(T \times\{h g\}) \quad \text { and } \quad V_{2}:=T_{x}(T \times\{h\}) .
$$

Let us also recall the map (37) and the fact (36). These last two sentences combine with Proposition A5.3 to yield

$$
D\left(\nu_{\mathcal{C}}, \rho_{\mathcal{C}^{\prime}}\right)_{x}=\Phi_{x}\left(\left(\nu_{\mathcal{C}}\right)_{x} \otimes\left(\rho_{\mathcal{C}^{\prime}}\right)_{x}\right)=\Phi_{x}^{V_{1}, V_{2}}\left(\left(\nu_{\mathcal{C}}\right)_{x} \otimes\left(\rho_{\mathcal{C}^{\prime}}\right)_{x}\right),
$$

where

$$
\Phi_{x}^{V_{1}, V_{2}}:\left.\left|T_{x} R\left(\Lambda_{\mathcal{C}}\right)^{\frac{1}{2}} \otimes\right| T_{x} \Lambda_{\mathcal{C}^{\prime}}\right|^{\frac{1}{2}} \longrightarrow\left|T_{x}\left(R\left(\Lambda_{\mathcal{C}}\right) \cap \Lambda_{\mathcal{C}^{\prime}}\right)\right|
$$

is defined in (66) and (67). One uses this definition along with (43), (44), Proposition 5.2.2, and Proposition 5.2 .5 to obtain

$$
D\left(\nu_{\mathcal{C}}, \rho_{\mathcal{C}^{\prime}}\right)_{x}\left(\left(\widetilde{\eta_{1}}\right)_{x}, \ldots,\left(\widetilde{\eta_{n-r}}\right)_{x}\right)=a \cdot\left|\Omega_{k \beta}\left(\eta_{1}, \ldots, \eta_{n-r}\right)\right|^{\frac{1}{2}}\left|\Omega_{k \beta^{\prime}}\left(\eta_{1}, \ldots, \eta_{n-r}\right)\right|^{\frac{1}{2}},
$$

where $a \in \mathbb{C}$ is defined as follows: let $\rho$ be the half-density in Lemma 5.4 .3 and define $a \in \mathbb{C}$ to be its image under the composition of (40) and (41). By Lemmas 5.4.3 and 5.4.4, this image is precisely $\kappa(G)$. We conclude that

$$
\begin{aligned}
D\left(\nu_{\mathcal{C}}, \rho_{\mathcal{C}^{\prime}}\right)_{x}\left(\left(\widetilde{\eta_{1}}\right)_{x}, \ldots,\left(\widetilde{\eta_{n-r}}\right)_{x}\right) & =\kappa(G) \cdot\left|\Omega_{k \beta}\left(\eta_{1}, \ldots, \eta_{n-r}\right)\right|^{\frac{1}{2}}\left|\Omega_{k \beta^{\prime}}\left(\eta_{1}, \ldots, \eta_{n-r}\right)\right|^{\frac{1}{2}} \\
& =k^{n-r} \kappa(G) \cdot\left|\Omega_{\beta}\left(\eta_{1}, \ldots, \eta_{n-r}\right)\right|^{\frac{1}{2}}\left|\Omega_{\beta^{\prime}}\left(\eta_{1}, \ldots, \eta_{n-r}\right)\right|^{\frac{1}{2}},
\end{aligned}
$$

completing the proof.

Fix $w \in W$ and note that $T$ is the $G$-stabilizer of $z_{w} \in R\left(\Lambda_{\mathcal{C}}\right) \cap \Lambda_{\mathcal{C}^{\prime}}$. It follows that

$$
\varphi_{w}: G / T \longrightarrow R\left(\Lambda_{\mathcal{C}}\right) \cap \Lambda_{\mathcal{C}^{\prime}}, \quad[g] \mapsto g \cdot z_{w}, \quad[g] \in G / T
$$

is a well-defined embedding of $G$-manifolds with image equal to the connected component $\Gamma_{w} \subseteq$ $R\left(\Lambda_{\mathcal{C}}\right) \cap \Lambda_{\mathcal{C}^{\prime}}$ (see Section 5.1). Let $\left(\varphi_{w}\right)^{*} D\left(\nu_{\mathcal{C}}, \rho_{\mathcal{C}^{\prime}}\right)$ denote the density on $G / T$ obtained by pulling $D\left(\nu_{\mathcal{C}}, \rho_{\mathcal{C}^{\prime}}\right)$ back along $\varphi_{w}$. Using the notation set just prior to Lemma 3.2.2, one describes this new density as follows.

Proposition 5.5.2. If $w \in W$, then

$$
\left(\varphi_{w}\right)^{*} D\left(\nu_{\mathcal{C}}, \rho_{\mathcal{C}^{\prime}}\right)=k^{n-r} \kappa(G) \cdot\left|\Xi_{\beta}\right|^{\frac{1}{2}}\left|\Xi_{\beta^{\prime}}\right|^{\frac{1}{2}} .
$$

Proof. Lemma 5.4 .1 and the $G$-equivariance of $\varphi_{w}$ imply that the density $\left(\varphi_{w}\right)^{*} D\left(\nu_{\mathcal{C}}, \rho_{\mathcal{C}^{\prime}}\right)$ is $G$ invariant. Since the density $\kappa(G) \cdot\left|\Xi_{\beta}\right|^{\frac{1}{2}}\left|\Xi_{\beta^{\prime}}\right|^{\frac{1}{2}}$ is also $G$-invariant, we are reduced to verifying (46) at $[e] \in G / T$. Now choose a basis $\left\{\eta_{1}, \ldots, \eta_{n-r}\right\}$ of $\mathfrak{t}^{\perp}$. Identify $T_{[e]}(G / T)$ with $\mathfrak{t}^{\perp}$ in the usual way, noting that $\left(\left(\varphi_{w}\right)^{*} D\left(\nu_{\mathcal{C}}, \rho_{\mathcal{C}^{\prime}}\right)\right)_{[e]}$ and $\kappa(G) \cdot\left|\left(\Xi_{\beta}\right)_{[e]}\right|^{\frac{1}{2}}\left|\left(\Xi_{\beta^{\prime}}\right)_{[e]}\right|^{\frac{1}{2}}$ are then densities on $\mathfrak{t}^{\perp}$. Our task is to verify that

$$
\left(\left(\varphi_{w}\right)^{*} D\left(\nu_{\mathcal{C}}, \rho_{\mathcal{C}^{\prime}}\right)\right)_{[e]}\left(\eta_{1}, \ldots, \eta_{n-r}\right)=\kappa(G) \cdot\left|\left(\Xi_{\beta}\right)_{[e]}\left(\eta_{1}, \ldots, \eta_{n-r}\right)\right|^{\frac{1}{2}}\left|\left(\Xi_{\beta^{\prime}}\right)_{[e]}\left(\eta_{1}, \ldots, \eta_{n-r}\right)\right|^{\frac{1}{2}} .
$$

Consider the differential of $\varphi_{w}$ at $[e]$, i.e. the vector space isomorphism

$$
\left(d \varphi_{w}\right)_{[e]}: \mathfrak{t}^{\perp} \stackrel{\cong}{\longrightarrow} T_{x}\left(R\left(\Lambda_{\mathcal{C}}\right) \cap \Lambda_{\mathcal{C}^{\prime}}\right)
$$

with $x:=z_{w}$. One readily verifies that

$$
\left(d \varphi_{w}\right)_{[e]}\left(\eta_{j}\right)=\left(\widetilde{\eta_{j}}\right)_{x}
$$


for all $j \in\{1, \ldots, n-r\}$. Hence

$$
\begin{aligned}
\left(\left(\varphi_{w}\right)^{*} D\left(\nu_{\mathcal{C}}, \rho_{\mathcal{C}^{\prime}}\right)\right)_{[e]}\left(\eta_{1}, \ldots, \eta_{n-r}\right) & =D\left(\nu_{\mathcal{C}}, \rho_{\mathcal{C}^{\prime}}\right)_{x}\left(\left(\widetilde{\eta_{1}}\right)_{x}, \ldots,\left(\widetilde{\eta_{n-r}}\right)_{x}\right) \\
& =k^{n-r} \kappa(G) \cdot\left|\left(\Xi_{\beta}\right)_{[e]}\left(\eta_{1}, \ldots, \eta_{n-r}\right)\right|^{\frac{1}{2}}\left|\left(\Xi_{\beta^{\prime}}\right)_{[e]}\left(\eta_{1}, \ldots, \eta_{n-r}\right)\right|^{\frac{1}{2}},
\end{aligned}
$$

where the last line follows from Proposition 5.5.1(ii).

We may now formulate and prove the main result of this section. To this end, recall the definition of the BKS pairing given in Section 2.4. This definition continues to make sense for a quasiHamiltonian $G$-space and cleanly intersecting quasi-Hamiltonian Lagrangian submanifolds thereof. One thereby obtains the following Lie-theoretic formula for the BKS pairing of $\left(R\left(\Lambda_{\mathcal{C}}\right), \nu_{\mathcal{C}}, \vartheta_{\mathcal{C}}\right)$ and $\left(\Lambda_{\mathcal{C}^{\prime}}, \rho_{\mathcal{C}^{\prime}}, \psi_{\mathcal{C}^{\prime}}\right)$.

Theorem 5.5.3. The BKS pairing of $\left(R\left(\Lambda_{\mathcal{C}}\right), \nu_{\mathcal{C}}, \vartheta_{\mathcal{C}}\right)$ and $\left(\Lambda_{\mathcal{C}^{\prime}}, \rho_{\mathcal{C}^{\prime}}, \psi_{\mathcal{C}^{\prime}}\right)$ is given by

$$
\operatorname{BKS}\left(\left(R\left(\Lambda_{\mathcal{C}}\right), \nu_{\mathcal{C}}, \vartheta_{\mathcal{C}}\right),\left(\Lambda_{\mathcal{C}^{\prime}}, \rho_{\mathcal{C}^{\prime}}, \psi_{\mathcal{C}^{\prime}}\right)\right)=k^{n-r} C(G, T) \cdot\left(\prod_{\alpha \in \Phi_{+}} \alpha(\beta) \alpha\left(\beta^{\prime}\right)\right)^{\frac{1}{2}}\left(\sum_{w \in W} e^{2 \pi i \mid w \beta-\beta^{\prime} \|^{2}}\right),
$$

where $C(G, T) \in \mathbb{R}$ is a constant depending only on the root system of $(G, T), \Phi_{+} \subseteq \mathfrak{t}^{*}$ is the set of positive roots, and $\mid w \beta-\beta^{\prime} \|$ is the length of $w \beta-\beta^{\prime}$ with respect to $\langle\cdot, \cdot\rangle$.

Proof. Recall the $G$-orbit decomposition of $R\left(\Lambda_{\mathcal{C}}\right) \cap \Lambda_{\mathcal{C}^{\prime}}$ given in Proposition [5.1.1, and the fact that $\vartheta_{\mathcal{C}}$ and $\psi_{\mathcal{C}^{\prime}}$ are $G$-invariant. We also observe that

$$
\vartheta_{\mathcal{C}}\left(z_{w}\right)=\psi_{\mathcal{C}}\left(R^{-1}\left(z_{w}\right)\right)=\psi_{\mathcal{C}}\left(\exp \left(w \beta-\beta^{\prime}\right), \exp (w \beta)\right)=e^{2 \pi i\left\langle w \beta, w \beta-\beta^{\prime}\right\rangle}
$$

and

$$
\psi_{\mathcal{C}^{\prime}}\left(z_{w}\right)=\psi_{\mathcal{C}}\left(\exp \left(w \beta-\beta^{\prime}\right), \exp \left(\beta^{\prime}\right)\right)=e^{2 \pi i\left\langle\beta^{\prime}, w \beta-\beta^{\prime}\right\rangle}
$$

for all $w \in W$. These last two sentences imply that $\vartheta_{\mathcal{C}}$ (resp. $\psi_{\mathcal{C}^{\prime}}$ ) takes the constant value $e^{2 \pi i\left\langle w \beta, w \beta-\beta^{\prime}\right\rangle}$ (resp. $\left.e^{2 \pi i\left\langle\beta^{\prime}, w \beta-\beta^{\prime}\right\rangle}\right)$ on $\Gamma_{w}$ for each $w \in W$. A straightforward computation now reveals that $\vartheta_{\mathcal{C}} \overline{\psi_{\mathcal{C}^{\prime}}}$ takes the constant value $e^{2 \pi i \mid w \beta-\beta^{\prime} \|^{2}}$ on $\Gamma_{w}$ for each $w \in W$.

Now note that

$$
\begin{aligned}
& \operatorname{BKS}\left(\left(R\left(\Lambda_{\mathcal{C}}\right), \nu_{\mathcal{C}}, \vartheta_{\mathcal{C}}\right),\left(\Lambda_{\mathcal{C}^{\prime}}, \rho_{\mathcal{C}^{\prime}}, \psi_{\mathcal{C}^{\prime}}\right)\right) \\
& =\int_{R\left(\beta_{\mathcal{C}}\right) \cap \Lambda_{\mathcal{C}^{\prime}}} \vartheta_{\mathcal{C}} \overline{\psi_{\mathcal{C}^{\prime}}} D\left(\nu_{\mathcal{C}}, \rho_{\mathcal{C}^{\prime}}\right) \\
& =\sum_{w \in W} \int_{\Gamma_{w}} \vartheta_{\mathcal{C}} \overline{\psi_{\mathcal{C}^{\prime}}} D\left(\nu_{\mathcal{C}}, \rho_{\mathcal{C}^{\prime}}\right) \quad(\text { by Proposition 5.1.1 and Corollary 5.1.2. } \\
& =\sum_{w \in W} e^{2 \pi i \mid w \beta-\beta^{\prime} \|^{2}} \int_{\Gamma_{w}} D\left(\nu_{\mathcal{C}}, \rho_{\mathcal{C}^{\prime}}\right) \quad \text { (by the previous paragraph) } \\
& =\sum_{w \in W} e^{2 \pi i \mid w \beta-\beta^{\prime} \|^{2}} \int_{G / T}\left(\varphi_{w}\right)^{*} D\left(\nu_{\mathcal{C}}, \rho_{\mathcal{C}^{\prime}}\right) \quad(\text { see }(\underline{45})) \\
& =k^{n-r} \kappa(G)\left(\int_{G / T}\left|\Xi_{\beta}\right|^{\frac{1}{2}}\left|\Xi_{\beta^{\prime}}\right|^{\frac{1}{2}}\right)\left(\sum_{w \in W} e^{2 \pi i \mid w \beta-\beta^{\prime} \|^{2}}\right) \quad \text { (by Proposition [5.5.2) }
\end{aligned}
$$




$$
\left.=k^{n-r} \kappa(G) \frac{\left(\prod_{\alpha \in \Phi_{+}} \alpha(\beta) \alpha\left(\beta^{\prime}\right)\right)^{\frac{1}{2}}}{\prod_{\alpha \in \Phi_{+}}\langle\alpha, \rho\rangle}\left(\sum_{w \in W} e^{2 \pi i \mid w \beta-\beta^{\prime} \|^{2}}\right) \quad \text { (by Lemma [3.2.2) }\right) .
$$

It just remains to observe that the real number

$$
\frac{\kappa(G)}{\prod_{\alpha \in \Phi_{+}}\langle\alpha, \rho\rangle}
$$

depends only on the root system of $(G, T)$.

\section{Appendix: Aspects of the BKS HALF-DEnsity}

A1. Some preliminaries. Let $V$ be an $n$-dimensional real vector space and fix a positive real number $\alpha$. Recall that a density of order $\alpha$ on $V$ is a map $\rho: V^{\oplus n} \longrightarrow \mathbb{C}$ satisfying

$$
\rho\left(A\left(v_{1}\right), \ldots, A\left(v_{n}\right)\right)=|\operatorname{det}(A)|^{\alpha} \rho\left(v_{1}, \ldots, v_{n}\right)
$$

for all $\left(v_{1}, \ldots, v_{n}\right) \in V^{\oplus n}$ and $A \in \operatorname{End}(V)$. Pointwise addition and scalar multiplication yield a complex vector space structure on the set of densities of order $\alpha$, and we let $|V|^{\alpha}$ denote the resulting complex vector space. Note that if $\left\{v_{1}, \ldots, v_{n}\right\}$ is a basis of $V$, then each $\rho \in|V|^{\alpha}$ is completely determined by the complex number $\rho\left(v_{1}, \ldots, v_{n}\right)$. It follows that $|V|^{\alpha}$ is one-dimensional for all $d$.

Now let $V$ be as above and suppose that $\alpha_{1}$ and $\alpha_{2}$ are positive real numbers. We may consider the pointwise product of $\rho_{1} \in|V|^{\alpha_{1}}$ and $\rho_{2} \in|V|^{\alpha_{2}}$, i.e. the map

$$
\rho_{1} \rho_{2}: V^{\oplus n} \longrightarrow \mathbb{C}, \quad\left(v_{1}, \ldots, v_{n}\right) \mapsto \rho_{1}\left(v_{1}, \ldots, v_{n}\right) \rho_{2}\left(v_{1}, \ldots, v_{n}\right), \quad\left(v_{1}, \ldots, v_{n}\right) \in V^{\oplus n} .
$$

One readily verifies that $\rho_{1} \rho_{2} \in|V|^{\alpha_{1}+\alpha_{2}}$, and that we have an isomorphism

$$
|V|^{\alpha_{1}} \otimes|V|^{\alpha_{2}} \stackrel{\cong}{\longrightarrow}|V|^{\alpha_{1}+\alpha_{2}}, \quad \rho_{1} \otimes \rho_{2} \mapsto \rho_{1} \rho_{2}, \quad \rho_{1} \in|V|^{\alpha_{1}}, \rho_{2} \in|V|^{\alpha_{2}} .
$$

One uses the term density (resp. half-density) in reference to a density of order 1 (resp. $\frac{1}{2}$ ) and writes $|V|$ for $|V|^{\alpha}$ when $\alpha=1$. The previous paragraph then specializes to yield an isomorphism

$$
|V|^{\frac{1}{2}} \otimes|V|^{\frac{1}{2}} \cong|V|, \quad \rho \otimes \rho^{\prime} \longrightarrow \rho \rho^{\prime}, \quad \rho, \rho^{\prime} \in|V|^{\frac{1}{2}} .
$$

Let $\phi: V \longrightarrow V^{\prime}$ be an isomorphism of $n$-dimensional real vector spaces, and fix a positive real number $\alpha$. Given any $\rho \in\left|V^{\prime}\right|^{\alpha}$, define $\phi^{*}(\rho) \in|V|^{\alpha}$ by the formula

$$
\phi^{*}(\rho)\left(v_{1}, \ldots, v_{n}\right)=\rho\left(\phi\left(v_{1}\right), \ldots, \phi\left(v_{n}\right)\right), \quad\left(v_{1}, \ldots, v_{n}\right) \in V^{\oplus n} .
$$

This defines a complex vector space isomorphism

$$
\phi^{*}:\left|V^{\prime}\right|^{\alpha} \stackrel{\cong}{\longrightarrow}|V|^{\alpha} .
$$

A2. Exact sequences. Suppose that one has an exact sequence

$$
0 \longrightarrow U \stackrel{i}{\longrightarrow} V \stackrel{j}{\longrightarrow} W \longrightarrow 0
$$

of finite-dimensional real vector spaces. This sequence canonically determines a vector space isomorphism

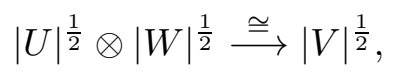

described as follows. Fix half-densities $\rho_{U} \in|U|^{\frac{1}{2}}$ and $\rho_{W} \in|W|^{\frac{1}{2}}$, and let $\rho_{V}$ denote the image of $\rho_{U} \otimes \rho_{W}$ under (48). To define $\rho_{V}$, we choose a subspace $W^{\prime} \subseteq V$ with the property that 
$V=\operatorname{ker}(j) \oplus W^{\prime}$. It follows that $\left.j\right|_{W^{\prime}}: W^{\prime} \longrightarrow W$ is an isomorphism. Choose bases $\left\{u_{1}, \ldots, u_{m}\right\}$ and $\left\{w_{1}, \ldots, w_{n}\right\}$ for $U$ and $W$, respectively, noting that

$$
\left\{v_{1}, \ldots, v_{m+n}\right\}:=\left\{i\left(u_{1}\right), \ldots, i\left(u_{m}\right),\left(\left.j\right|_{W^{\prime}}\right)^{-1}\left(w_{1}\right), \ldots,\left(\left.j\right|_{W^{\prime}}\right)^{-1}\left(w_{n}\right)\right\}
$$

is a basis of $V$. One then has

$$
\rho_{V}\left(v_{1}, \ldots, v_{m+n}\right)=\rho_{U}\left(u_{1}, \ldots, u_{m}\right) \rho_{W}\left(w_{1}, \ldots, w_{n}\right),
$$

a condition that completely determines $\rho_{V}$.

Lemma A2.1. Suppose that $U, V$, and $W$ are finite-dimensional real vector spaces, and that we have two exact sequences

$$
0 \longrightarrow U \stackrel{i}{\longrightarrow} V \stackrel{j}{\longrightarrow} W \longrightarrow 0 \quad \text { and } \quad 0 \longrightarrow U \stackrel{i^{\prime}}{\longrightarrow} V \stackrel{j^{\prime}}{\longrightarrow} W \longrightarrow 0 .
$$

Let

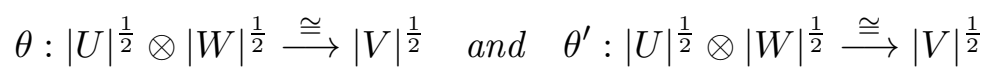

be the isomorphisms induced by the first and second sequences, respectively. Let $k: V \longrightarrow V$ be an isomorphism that makes the squares in

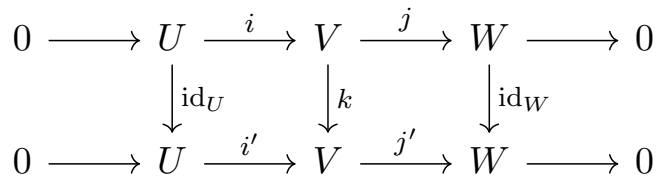

commute, where $\operatorname{id}_{U}$ and $\operatorname{id}_{W}$ are the identity maps. We then have

$$
\theta=|\operatorname{det}(k)|^{\frac{1}{2}} \theta^{\prime} .
$$

Proof. Let $\rho_{U} \in|U|^{\frac{1}{2}}$ and $\rho_{W} \in|W|^{\frac{1}{2}}$ be given, and set $\rho_{V}:=\theta\left(\rho_{U} \otimes \rho_{W}\right)$ and $\rho_{V}^{\prime}:=\theta^{\prime}\left(\rho_{U} \otimes \rho_{W}\right)$. Our task is to prove that

$$
\rho_{V}=|\operatorname{det}(k)|^{\frac{1}{2}} \rho_{V}^{\prime} .
$$

We begin by choosing a complement $C$ of $\operatorname{ker}(j)$ in $V$, noting that $C^{\prime}:=k(C)$ is a complement of $\operatorname{ker}\left(j^{\prime}\right)$ in $V$. Let us also choose bases $\left\{u_{1}, \ldots, u_{m}\right\}$ and $\left\{w_{1}, \ldots, w_{n}\right\}$ of $U$ and $W$, respectively. We then have bases

$$
\left\{v_{1}, \ldots, v_{m+n}\right\}:=\left\{i\left(u_{1}\right), \ldots, i\left(u_{m}\right),\left(\left.j\right|_{C}\right)^{-1}\left(w_{1}\right), \ldots,\left(\left.j\right|_{C}\right)^{-1}\left(w_{n}\right)\right\}
$$

and

$$
\left\{v_{1}^{\prime}, \ldots, v_{m+n}^{\prime}\right\}:=\left\{i^{\prime}\left(u_{1}\right), \ldots, i^{\prime}\left(u_{m}\right),\left(\left.j^{\prime}\right|_{C^{\prime}}\right)^{-1}\left(w_{1}\right), \ldots,\left(\left.j^{\prime}\right|_{C^{\prime}}\right)^{-1}\left(w_{n}\right)\right\}
$$

of $V$. Our description of (48) implies that

$$
\rho_{V}\left(v_{1}, \ldots, v_{n+m}\right)=\rho_{U}\left(u_{1}, \ldots, u_{m}\right) \rho_{W}\left(w_{1}, \ldots, w_{n}\right)=\rho_{V}^{\prime}\left(v_{1}^{\prime}, \ldots, v_{m+n}^{\prime}\right) .
$$

At the same time, note that

$$
v_{p}^{\prime}=i^{\prime}\left(u_{p}\right)=k\left(i\left(u_{p}\right)\right)=k\left(v_{p}\right)
$$

for all $p \in\{1, \ldots, m\}$. We also have

$$
j^{\prime}\left(k\left(v_{m+q}\right)\right)=j^{\prime}\left(k\left(\left(\left.j\right|_{C}\right)^{-1}\left(w_{q}\right)\right)\right)=j\left(\left(\left.j\right|_{C}\right)^{-1}\left(w_{q}\right)\right)=w_{q}
$$

for all $q \in\{1, \ldots, n\}$, and we note that $k\left(v_{m+q}\right) \in C^{\prime}$ for all $q \in\{1, \ldots, n\}$. This last sentence tells us that

$$
k\left(v_{m+q}\right)=\left(\left.j^{\prime}\right|_{C^{\prime}}\right)^{-1}\left(w_{q}\right)=v_{m+q}^{\prime}
$$


for all $q \in\{1, \ldots, n\}$. Hence

$$
\begin{aligned}
\rho_{V}\left(v_{1}, \ldots, v_{n+m}\right) & =\rho_{V}^{\prime}\left(v_{1}^{\prime}, \ldots, v_{n+m}^{\prime}\right) & & {[\text { by (49)] }} \\
& =\rho_{V}^{\prime}\left(k\left(v_{1}\right), \ldots, k\left(v_{m+n}\right)\right) & & {[\text { by (50) and (51)] }] } \\
& =|\operatorname{det}(k)|^{\frac{1}{2}} \rho_{V}^{\prime}\left(v_{1}, \ldots, v_{m+n}\right) . & &
\end{aligned}
$$

Since $\left\{v_{1}, \ldots, v_{m+n}\right\}$ is a basis of $V$, this establishes that $\rho_{V}=|\operatorname{det}(k)|^{\frac{1}{2}} \rho_{V}^{\prime}$.

One also has the following result. Its proof is straightforward and omitted.

Lemma A2.2. Suppose that

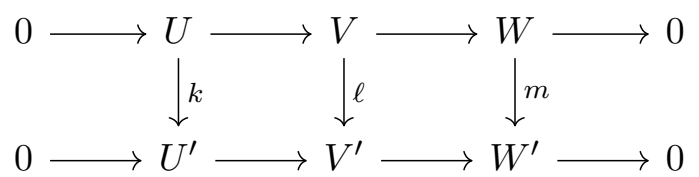

is a diagram of finite-dimensional real vector spaces and linear maps. Assume that the rows are exact, the vertical maps are isomorphisms, and that the two squares commute. Let

$$
\theta:|U|^{\frac{1}{2}} \otimes|W|^{\frac{1}{2} \cong} \cong|V|^{\frac{1}{2}} \quad \text { and } \quad \theta^{\prime}:\left|U^{\prime}\right|^{\frac{1}{2}} \otimes\left|W^{\prime}\right|^{\frac{1}{2}} \cong\left|V^{\prime}\right|^{\frac{1}{2}}
$$

be the isomorphisms induced by the top and bottom rows, respectively. The diagram

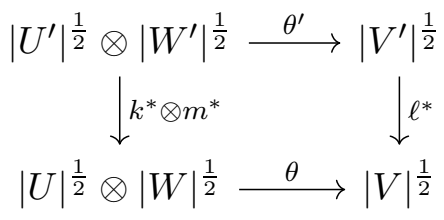

then commutes.

Lemma A2.3. Suppose that

$$
0 \longrightarrow U \stackrel{i}{\longrightarrow} V \stackrel{j}{\longrightarrow} W \longrightarrow 0 \quad \text { and } \quad 0 \longrightarrow U^{\prime} \stackrel{i^{\prime}}{\longrightarrow} V^{\prime} \stackrel{j^{\prime}}{\longrightarrow} W^{\prime} \longrightarrow 0
$$

are exact sequences of finite-dimensional real vector spaces and linear maps. Denote by

$$
\theta:|U|^{\frac{1}{2}} \otimes|W|^{\frac{1}{2} \cong} \cong|V|^{\frac{1}{2}} \quad \text { and } \quad \theta^{\prime}:\left|U^{\prime}\right|^{\frac{1}{2}} \otimes\left|W^{\prime}\right|^{\frac{1}{2}} \cong\left|V^{\prime}\right|^{\frac{1}{2}}
$$

the isomorphisms induced by the first and second sequences, respectively. Now consider the exact sequence

$$
0 \longrightarrow U \oplus U^{\prime} \stackrel{i \oplus i^{\prime}}{\longrightarrow} V \oplus V^{\prime} \stackrel{j \oplus j^{\prime}}{\longrightarrow} W \oplus W^{\prime} \longrightarrow 0
$$

and the induced isomorphism

$$
\theta^{\prime \prime}:\left|U \oplus U^{\prime}\right|^{\frac{1}{2}} \otimes\left|W \oplus W^{\prime}\right|^{\frac{1}{2}} \stackrel{\cong}{\longrightarrow}\left|V \oplus V^{\prime}\right|^{\frac{1}{2}} .
$$

Let us also consider the canonical isomorphisms

$\xi:|U|^{\frac{1}{2}} \otimes\left|U^{\prime}\right|^{\frac{1}{2} \cong} \cong\left|U \oplus U^{\prime}\right|^{\frac{1}{2}}, \quad \eta:|W|^{\frac{1}{2}} \otimes\left|W^{\prime}\right|^{\frac{1}{2} \cong} \cong\left|W \oplus W^{\prime}\right|^{\frac{1}{2}}, \quad$ and $\quad \zeta:|V|^{\frac{1}{2}} \otimes\left|V^{\prime}\right|^{\frac{1}{2}} \cong\left|V \oplus V^{\prime}\right|^{\frac{1}{2}}$.

We then have a commutative diagram

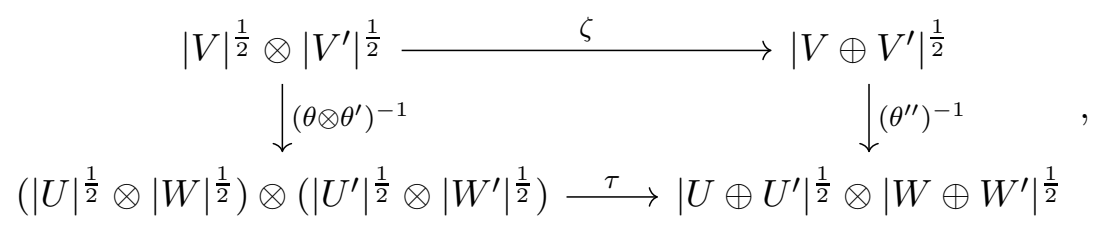


where $\tau$ is the composite map

$\left(|U|^{\frac{1}{2}} \otimes|W|^{\frac{1}{2}}\right) \otimes\left(\left|U^{\prime}\right|^{\frac{1}{2}} \otimes\left|W^{\prime}\right|^{\frac{1}{2}}\right) \stackrel{\cong}{\longrightarrow}\left(\left.\left|U^{\frac{1}{2}} \otimes\right| U^{\prime}\right|^{\frac{1}{2}}\right) \otimes\left(|W|^{\frac{1}{2}} \otimes\left|W^{\prime}\right|^{\frac{1}{2}}\right) \stackrel{\xi \otimes \eta}{\longrightarrow}\left|U \oplus U^{\prime}\right|^{\frac{1}{2}} \otimes\left|W \oplus W^{\prime}\right|^{\frac{1}{2}}$.

Proof. It suffices to prove that $\zeta \circ\left(\theta \otimes \theta^{\prime}\right)=\theta^{\prime \prime} \circ \tau$. To this end, choose bases $\left\{u_{1}, \ldots, u_{m}\right\}$, $\left\{u_{1}^{\prime}, \ldots, u_{n}^{\prime}\right\},\left\{w_{1}, \ldots, w_{p}\right\}$, and $\left\{w_{1}^{\prime}, \ldots, w_{q}^{\prime}\right\}$ of $U, U^{\prime}, W$, and $W^{\prime}$, respectively. Let $\left\{v_{1}, \ldots, v_{m+p}\right\}$ be a basis of $V$ satisfying $v_{k}=i\left(u_{k}\right)$ for all $k \in\{1, \ldots, m\}$ and $j\left(v_{\ell+m}\right)=w_{\ell}$ for all $\ell \in\{1, \ldots, p\}$. We may analogously find a basis $\left\{v_{1}^{\prime}, \ldots, v_{n+q}^{\prime}\right\}$ of $V^{\prime}$ satisfying $v_{k}^{\prime}=i^{\prime}\left(u_{k}^{\prime}\right)$ for all $k \in\{1, \ldots, n\}$ and $j^{\prime}\left(v_{\ell+n}\right)=w_{\ell}^{\prime}$ for all $\ell \in\{1, \ldots, q\}$. Observe that

$$
\mathcal{B}:=\left\{\left(v_{1}, 0\right), \ldots,\left(v_{m+p}, 0\right),\left(0, v_{1}^{\prime}\right), \ldots,\left(0, v_{n+q}^{\prime}\right)\right\}
$$

is a basis of $V \oplus V^{\prime}$, and that a suitable reordering of basis vectors produces the alternative basis

$$
\mathcal{B}^{\prime}:=\left\{\left(v_{1}, 0\right), \ldots,\left(v_{m}, 0\right),\left(0, v_{1}^{\prime}\right), \ldots,\left(0, v_{n}^{\prime}\right),\left(v_{m+1}, 0\right), \ldots,\left(v_{m+p}, 0\right),\left(0, v_{n+1}^{\prime}\right), \ldots,\left(0, v_{n+q}^{\prime}\right)\right\} .
$$

Now suppose that $\rho \in|U|^{\frac{1}{2}}, \rho^{\prime} \in\left|U^{\prime}\right|^{\frac{1}{2}}, \nu \in|W|^{\frac{1}{2}}$, and $\nu^{\prime} \in\left|W^{\prime}\right|^{\frac{1}{2}}$. If we regard $\mathcal{B}$ and $\mathcal{B}^{\prime}$ as tuples of vectors in $V \oplus V^{\prime}$, then

$$
\begin{aligned}
\left(\zeta \circ\left(\theta \otimes \theta^{\prime}\right)\right)\left(\rho \otimes \nu \otimes \rho^{\prime} \otimes \nu^{\prime}\right)(\mathcal{B}) & =\theta(\rho \otimes \nu)\left(v_{1}, \ldots, v_{m+p}\right) \theta^{\prime}\left(\rho^{\prime} \otimes \nu^{\prime}\right)\left(v_{1}^{\prime}, \ldots, v_{n+q}^{\prime}\right) \\
& =\rho\left(u_{1}, \ldots, u_{m}\right) \nu\left(w_{1}, \ldots, w_{p}\right) \rho^{\prime}\left(u_{1}^{\prime}, \ldots, u_{n}^{\prime}\right) \nu^{\prime}\left(w_{1}^{\prime}, \ldots, w_{q}^{\prime}\right)
\end{aligned}
$$

and

$$
\begin{aligned}
& \left(\theta^{\prime \prime} \circ \tau\right)\left(\rho \otimes \nu \otimes \rho^{\prime} \otimes \nu^{\prime}\right)\left(\mathcal{B}^{\prime}\right) \\
& =\xi\left(\rho \otimes \rho^{\prime}\right)\left(\left(u_{1}, 0\right), \ldots,\left(u_{m}, 0\right),\left(0, u_{1}^{\prime}\right), \ldots,\left(0, u_{n}^{\prime}\right)\right) \eta\left(\nu \otimes \nu^{\prime}\right)\left(\left(w_{1}, 0\right), \ldots,\left(w_{p}, 0\right),\left(0, w_{1}^{\prime}\right), \ldots,\left(0, w_{q}^{\prime}\right)\right) \\
& =\rho\left(u_{1}, \ldots, u_{m}\right) \rho^{\prime}\left(u_{1}^{\prime}, \ldots, u_{n}^{\prime}\right) \nu\left(w_{1}, \ldots, w_{p}\right) \nu^{\prime}\left(w_{1}^{\prime}, \ldots, w_{q}^{\prime}\right) .
\end{aligned}
$$

It follows that

$$
\left(\zeta \circ\left(\theta \otimes \theta^{\prime}\right)\right)\left(\rho \otimes \nu \otimes \rho^{\prime} \otimes \nu^{\prime}\right)(\mathcal{B})=\left(\theta^{\prime \prime} \circ \tau\right)\left(\rho \otimes \nu \otimes \rho^{\prime} \otimes \nu^{\prime}\right)\left(\mathcal{B}^{\prime}\right) .
$$

Since $\mathcal{B}^{\prime}$ is obtained by reordering the vectors in $\mathcal{B}$, this amounts to the statement

$$
\left(\zeta \circ\left(\theta \otimes \theta^{\prime}\right)\right)\left(\rho \otimes \nu \otimes \rho^{\prime} \otimes \nu^{\prime}\right)(\mathcal{B})=\left(\theta^{\prime \prime} \circ \tau\right)\left(\rho \otimes \nu \otimes \rho^{\prime} \otimes \nu^{\prime}\right)(\mathcal{B}) .
$$

We conclude that

$$
\left(\zeta \circ\left(\theta \otimes \theta^{\prime}\right)\right)\left(\rho \otimes \nu \otimes \rho^{\prime} \otimes \nu^{\prime}\right)=\left(\theta^{\prime \prime} \circ \tau\right)\left(\rho \otimes \nu \otimes \rho^{\prime} \otimes \nu^{\prime}\right)
$$

completing the proof.

A3. The BKS density. Let $M$ be a manifold equipped with an arbitrary two-form $\omega \in \Omega^{2}(M)$. Consider the open submanifold of points at which $\omega$ is non-degenerate, i.e.

$$
M^{\circ}:=\left\{m \in M: \omega_{m} \text { is non-degenerate }\right\} .
$$

It follows that $\left(T_{m} M, \omega_{m}\right)$ is a symplectic vector space for all $m \in M^{\circ}$. This suggests the following generalization of Lagrangian submanifolds in symplectic geometry (cf. Definition 4.1.3).

Definition A3.1. We call a submanifold $\Lambda \subseteq M$ Lagrangian if it satisfies the following conditions:

(i) $\Lambda \subseteq M^{\circ}$;

(ii) $T_{m} \Lambda$ is a Lagrangian subspace of $\left(T_{m} M, \omega_{m}\right)$ for all $m \in \Lambda$. 
Let $\Lambda_{1}, \Lambda_{2} \subseteq M$ be Lagrangian submanifolds that intersect cleanly in $M$. Fix $m \in \Lambda_{1} \cap \Lambda_{2}$, and let $T_{m} \Lambda_{1}+T_{m} \Lambda_{2}$ be the subspace of $T_{m} M$ generated by $T_{m} \Lambda_{1}$ and $T_{m} \Lambda_{2}$. One may restrict $\omega_{m}$ to a bilinear form on $T_{m} \Lambda_{1}+T_{m} \Lambda_{2}$, and easily verify that this restricted form descends to a symplectic

form on $\frac{T_{m} \Lambda_{1}+T_{m} \Lambda_{2}}{T_{m}\left(\Lambda_{1} \cap \Lambda_{2}\right)}$. This new symplectic form induces a half-density on $\frac{T_{m} \Lambda_{1}+T_{m} \Lambda_{2}}{T_{m}\left(\Lambda_{1} \cap \Lambda_{2}\right)}$, which in turn forms a basis of $\left|\frac{T_{m} \Lambda_{1}+T_{m} \Lambda_{2}}{T_{m}\left(\Lambda_{1} \cap \Lambda_{2}\right)}\right|^{\frac{1}{2}}$. We thereby obtain a canonical isomorphism

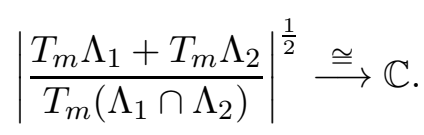

Now consider the exact sequence

$$
0 \longrightarrow T_{m}\left(\Lambda_{1} \cap \Lambda_{2}\right) \stackrel{\alpha}{\longrightarrow} T_{m} \Lambda_{1}+T_{m} \Lambda_{2} \stackrel{\beta}{\longrightarrow} \frac{T_{m} \Lambda_{1}+T_{m} \Lambda_{2}}{T_{m}\left(\Lambda_{1} \cap \Lambda_{2}\right)} \longrightarrow 0,
$$

where $\alpha$ is inclusion and $\beta$ is the quotient map. Let us also consider the external direct sum $T_{m} \Lambda_{1} \oplus T_{m} \Lambda_{2}$ of $T_{m} \Lambda_{1}$ and $T_{m} \Lambda_{2}$. The two linear maps

$$
\gamma: T_{m}\left(\Lambda_{1} \cap \Lambda_{2}\right) \longrightarrow T_{m} \Lambda_{1} \oplus T_{m} \Lambda_{2}, \quad v \mapsto(v, v), \quad v \in T_{m}\left(\Lambda_{1} \cap \Lambda_{2}\right)
$$

and

$$
\delta: T_{m} \Lambda_{1} \oplus T_{m} \Lambda_{2} \longrightarrow T_{m} \Lambda_{1}+T_{m} \Lambda_{2}, \quad\left(v_{1}, v_{2}\right) \mapsto v_{1}-v_{2}, \quad\left(v_{1}, v_{2}\right) \in T_{m} \Lambda_{1} \oplus T_{m} \Lambda_{2}
$$

then fit into an exact sequence

$$
0 \longrightarrow T_{m}\left(\Lambda_{1} \cap \Lambda_{2}\right) \stackrel{\gamma}{\longrightarrow} T_{m} \Lambda_{1} \oplus T_{m} \Lambda_{2} \stackrel{\delta}{\longrightarrow} T_{m} \Lambda_{1}+T_{m} \Lambda_{2} \longrightarrow 0 .
$$

We now observe that

$$
\begin{aligned}
& \left|T_{m} \Lambda_{1}\right|^{\frac{1}{2}} \otimes\left|T_{m} \Lambda_{2}\right|^{\frac{1}{2}} \cong\left|T_{m} \Lambda_{1} \oplus T_{m} \Lambda_{2}\right|^{\frac{1}{2}} \quad \text { [by (48)] } \\
& \text { (55b) } \cong\left|T_{m}\left(\Lambda_{1} \cap \Lambda_{2}\right)\right|^{\frac{1}{2}} \otimes\left|T_{m} \Lambda_{1}+T_{m} \Lambda_{2}\right|^{\frac{1}{2}} \quad \text { [by (448) and (54)] } \\
& \cong\left|T_{m}\left(\Lambda_{1} \cap \Lambda_{2}\right)\right|^{\frac{1}{2}} \otimes\left|T_{m}\left(\Lambda_{1} \cap \Lambda_{2}\right)\right|^{\frac{1}{2}} \otimes\left|\frac{T_{m} \Lambda_{1}+T_{m} \Lambda_{2}}{T_{m}\left(\Lambda_{1} \cap \Lambda_{2}\right)}\right|^{\frac{1}{2}}[\text { by (48) and (153) ] } \\
& \cong\left|T_{m}\left(\Lambda_{1} \cap \Lambda_{2}\right)\right| \quad \text { [by (152)]. }
\end{aligned}
$$

These considerations yield a canonical isomorphism

$$
\Phi_{m}:\left|T_{m} \Lambda_{1}\right|^{\frac{1}{2}} \otimes\left|T_{m} \Lambda_{2}\right|^{\frac{1}{2}} \stackrel{\cong}{\longrightarrow}\left|T_{m}\left(\Lambda_{1} \cap \Lambda_{2}\right)\right| .
$$

If $\rho_{1}$ and $\rho_{2}$ are half-densities on $\Lambda_{1}$ and $\Lambda_{2}$, respectively, then the formula

$$
D\left(\rho_{1}, \rho_{2}\right)_{m}:=\Phi_{m}\left(\left(\rho_{1}\right)_{m} \otimes\left(\rho_{2}\right)_{m}\right), \quad m \in \Lambda_{1} \cap \Lambda_{2}
$$

defines a density $D\left(\rho_{1}, \rho_{2}\right)$ on $\Lambda_{1} \cap \Lambda_{2}$.

Definition A3.2. One calls $D\left(\rho_{1}, \rho_{2}\right)$ the $B K S$ density on $\Lambda_{1} \cap \Lambda_{2}$ associated to $\rho_{1}$ and $\rho_{2}$. 
A4. Some technical results. Let $M$ be a manifold endowed with a two-form $\omega \in \Omega^{2}(M)$, and suppose that $\Lambda_{1}, \Lambda_{2} \subseteq M$ are cleanly intersecting Lagrangian submanifolds. Each fixed $m \in \Lambda_{1} \cap \Lambda_{2}$ has an associated inclusion $\theta: T_{m}\left(\Lambda_{1} \cap \Lambda_{2}\right) \oplus T_{m}\left(\Lambda_{1} \cap \Lambda_{2}\right) \longrightarrow T_{m} \Lambda_{1} \oplus T_{m} \Lambda_{2}$ and map

$$
\vartheta: T_{m} \Lambda_{1} \oplus T_{m} \Lambda_{2} \longrightarrow \frac{T_{m} \Lambda_{1}+T_{m} \Lambda_{2}}{T_{m}\left(\Lambda_{1} \cap \Lambda_{2}\right)}, \quad\left(v_{1}, v_{2}\right) \mapsto\left[v_{1}-v_{2}\right], \quad\left(v_{1}, v_{2}\right) \in T_{m} \Lambda_{1} \oplus T_{m} \Lambda_{2},
$$

where $\left[v_{1}-v_{2}\right]$ denotes the class of $v_{1}-v_{2}$ in $\frac{T_{m} \Lambda_{1}+T_{m} \Lambda_{2}}{T_{m}\left(\Lambda_{1} \cap \Lambda_{2}\right)}$. We then have an exact sequence

$$
0 \longrightarrow T_{m}\left(\Lambda_{1} \cap \Lambda_{2}\right) \oplus T_{m}\left(\Lambda_{1} \cap \Lambda_{2}\right) \stackrel{\theta}{\longrightarrow} T_{m} \Lambda_{1} \oplus T_{m} \Lambda_{2} \stackrel{\vartheta}{\longrightarrow} \frac{T_{m} \Lambda_{1}+T_{m} \Lambda_{2}}{T_{m}\left(\Lambda_{1} \cap \Lambda_{2}\right)}
$$

so that

(58a)

$$
\begin{aligned}
\left|T_{m} \Lambda_{1} \oplus T_{m} \Lambda_{2}\right|^{\frac{1}{2}} & \cong\left|T_{m}\left(\Lambda_{1} \cap \Lambda_{2}\right) \oplus T_{m}\left(\Lambda_{1} \cap \Lambda_{2}\right)\right|^{\frac{1}{2}} \otimes\left|\frac{T_{m} \Lambda_{1}+T_{m} \Lambda_{2}}{T_{m}\left(\Lambda_{1} \cap \Lambda_{2}\right)}\right|^{\frac{1}{2}} \quad \text { [by (48) and (57)] } \\
& \cong\left|T_{m}\left(\Lambda_{1} \cap \Lambda_{2}\right)\right|^{\frac{1}{2}} \otimes\left|T_{m}\left(\Lambda_{1} \cap \Lambda_{2}\right)\right|^{\frac{1}{2}} \otimes\left|\frac{T_{m} \Lambda_{1}+T_{m} \Lambda_{2}}{T_{m}\left(\Lambda_{1} \cap \Lambda_{2}\right)}\right|^{\frac{1}{2}} \quad \text { [by (48)] }
\end{aligned}
$$

Lemma A4.1. The isomorphism

$$
\left|T_{m} \Lambda_{1} \oplus T_{m} \Lambda_{2}\right|^{\frac{1}{2}} \stackrel{\cong}{\longrightarrow}\left|T_{m}\left(\Lambda_{1} \cap \Lambda_{2}\right)\right|^{\frac{1}{2}} \otimes\left|T_{m}\left(\Lambda_{1} \cap \Lambda_{2}\right)\right|^{\frac{1}{2}} \otimes\left|\frac{T_{m} \Lambda_{1}+T_{m} \Lambda_{2}}{T_{m}\left(\Lambda_{1} \cap \Lambda_{2}\right)}\right|^{\frac{1}{2}}
$$

obtained by composing (55b) and (55c) equals that obtained by composing (58a) and (58b).

Proof. Let us write

$$
\begin{aligned}
& \psi:\left|T_{m} \Lambda_{1} \oplus T_{m} \Lambda_{2}\right|^{\frac{1}{2}} \cong\left|T_{m}\left(\Lambda_{1} \cap \Lambda_{2}\right)\right|^{\frac{1}{2}} \otimes\left|T_{m} \Lambda_{1}+T_{m} \Lambda_{2}\right|^{\frac{1}{2}} \\
& \varphi:\left|T_{m}\left(\Lambda_{1} \cap \Lambda_{2}\right)\right|^{\frac{1}{2}} \otimes\left|T_{m} \Lambda_{1}+T_{m} \Lambda_{2}\right|^{\frac{1}{2}} \cong\left|T_{m}\left(\Lambda_{1} \cap \Lambda_{2}\right)\right|^{\frac{1}{2}} \otimes\left|T_{m}\left(\Lambda_{1} \cap \Lambda_{2}\right)\right|^{\frac{1}{2}} \otimes\left|\frac{T_{m} \Lambda_{1}+T_{m} \Lambda_{2}}{T_{m}\left(\Lambda_{1} \cap \Lambda_{2}\right)}\right|^{\frac{1}{2}} \\
& \psi^{\prime}:\left|T_{m} \Lambda_{1} \oplus T_{m} \Lambda_{2}\right|^{\frac{1}{2}} \stackrel{\cong}{\longrightarrow}\left|T_{m}\left(\Lambda_{1} \cap \Lambda_{2}\right) \oplus T_{m}\left(\Lambda_{1} \cap \Lambda_{2}\right)\right|^{\frac{1}{2}} \otimes\left|\frac{T_{m} \Lambda_{1}+T_{m} \Lambda_{2}}{T_{m}\left(\Lambda_{1} \cap \Lambda_{2}\right)}\right|^{\frac{1}{2}}, \text { and } \\
& \varphi^{\prime}:\left|T_{m}\left(\Lambda_{1} \cap \Lambda_{2}\right) \oplus T_{m}\left(\Lambda_{1} \cap \Lambda_{2}\right)\right|^{\frac{1}{2}} \otimes\left|\frac{T_{m} \Lambda_{1}+T_{m} \Lambda_{2}}{T_{m}\left(\Lambda_{1} \cap \Lambda_{2}\right)}\right|^{\frac{1}{2}} \stackrel{\cong}{\longrightarrow}\left|T_{m}\left(\Lambda_{1} \cap \Lambda_{2}\right)\right|^{\frac{1}{2}} \otimes\left|T_{m}\left(\Lambda_{1} \cap \Lambda_{2}\right)\right|^{\frac{1}{2}} \otimes\left|\frac{T_{m} \Lambda_{1}+T_{m} \Lambda_{2}}{T_{m}\left(\Lambda_{1} \cap \Lambda_{2}\right)}\right|^{\frac{1}{2}}
\end{aligned}
$$

for the isomorphisms (55b), (55c), (58a), and (58b), respectively. Our objective is to prove that $\varphi \circ \psi=\varphi^{\prime} \circ \psi^{\prime}$. In what follows, we verify the equivalent statement that $\psi^{-1} \circ \varphi^{-1}=\left(\psi^{\prime}\right)^{-1} \circ\left(\varphi^{\prime}\right)^{-1}$.

We begin by making a few choices, the first being a basis $\left\{u_{1}, \ldots, u_{m}\right\}$ of $T_{m}\left(\Lambda_{1} \cap \Lambda_{2}\right)$. Let us also consider the subspaces of $T_{m} \Lambda_{1} \oplus T_{m} \Lambda_{2}$ defined by $W^{\prime}:=T_{m}\left(\Lambda_{1} \cap \Lambda_{2}\right) \oplus\{0\}$ and $W^{\prime \prime}:=$ $\{0\} \oplus T_{m}\left(\Lambda_{1} \cap \Lambda_{2}\right)$. It follows that

$$
\left\{w_{1}^{\prime}, \ldots, w_{m}^{\prime}\right\}:=\left\{\left(u_{1}, 0\right), \ldots,\left(u_{m}, 0\right)\right\} \quad \text { and } \quad\left\{w_{1}^{\prime \prime}, \ldots, w_{m}^{\prime \prime}\right\}:=\left\{\left(0, u_{1}\right), \ldots,\left(0, u_{m}\right)\right\}
$$

are bases of $W^{\prime}$ and $W^{\prime \prime}$, respectively. At the same time, choose a complement $V_{1}$ (resp. $V_{2}$ ) of $T_{m}\left(\Lambda_{1} \cap \Lambda_{2}\right)$ in $T_{m} \Lambda_{1}$ (resp. $\left.T_{m} \Lambda_{2}\right)$ and set

$$
W:=V_{1} \oplus V_{2} \subseteq T_{m} \Lambda_{1} \oplus T_{m} \Lambda_{2} .
$$

Fix a basis $\left\{v_{1}, \ldots, v_{n}\right\}$ (resp. $\left\{\bar{v}_{1}, \ldots, \bar{v}_{n}\right\}$ ) of $V_{1}$ (resp. $V_{2}$ ), noting that

$$
\left\{w_{1}, \ldots, w_{2 n}\right\}:=\left\{\left(v_{1}, 0\right), \ldots,\left(v_{n}, 0\right),\left(0, \bar{v}_{1}\right), \ldots,\left(0, \bar{v}_{n}\right)\right\}
$$

is a basis of $W$. 
Let us now describe $\rho:=\left(\psi^{-1} \circ \varphi^{-1}\right)\left(\rho_{1} \otimes \rho_{2} \otimes \rho_{3}\right)$ for arbitrary $\rho_{1}, \rho_{2} \in\left|T_{m}\left(\Lambda_{1} \cap \Lambda_{2}\right)\right|^{\frac{1}{2}}$ and $\rho_{3} \in\left|\frac{T_{m} \Lambda_{1}+T_{m} \Lambda_{2}}{T_{m}\left(\Lambda_{1} \cap \Lambda_{2}\right)}\right|^{\frac{1}{2}}$. To this end, recall the map

$$
\delta: T_{m} \Lambda_{1} \oplus T_{m} \Lambda_{2} \longrightarrow T_{m} \Lambda_{1}+T_{m} \Lambda_{2}
$$

from Section A3. Note that $\delta$ identifies $W$ with a complement $\delta(W)$ of $T_{m}\left(\Lambda_{1} \cap \Lambda_{2}\right)$ in $T_{m} \Lambda_{1}+T_{m} \Lambda_{2}$. We thereby obtain

$$
\varphi^{-1}\left(\rho_{1} \otimes \rho_{2} \otimes \rho_{3}\right)=\rho_{1} \otimes \rho^{\prime}
$$

where $\rho^{\prime} \in\left|T_{m} \Lambda_{1}+T_{m} \Lambda_{2}\right|^{\frac{1}{2}}$ and

$$
\begin{aligned}
\rho^{\prime}\left(u_{1}, \ldots, u_{m}, \delta\left(w_{1}\right), \ldots, \delta\left(w_{2 n}\right)\right) & =\rho_{2}\left(u_{1}, \ldots, u_{m}\right) \rho_{3}\left(\beta\left(\delta\left(w_{1}\right)\right), \ldots, \beta\left(\delta\left(w_{2 n}\right)\right)\right) \\
& =\rho_{2}\left(u_{1}, \ldots, u_{m}\right) \rho_{3}\left(\vartheta\left(w_{1}\right), \ldots, \vartheta\left(w_{2 n}\right)\right) .
\end{aligned}
$$

We also observe that $W+W^{\prime \prime}$ is a complement of image $(\alpha)$ in $T_{m} \Lambda_{1} \oplus T_{m} \Lambda_{2}$, where

$$
\alpha: T_{m}\left(\Lambda_{1} \cap \Lambda_{2}\right) \longleftrightarrow T_{m} \Lambda_{1}+T_{m} \Lambda_{2}
$$

is the inclusion. Noting that $\rho:=\psi^{-1}\left(\rho_{1} \otimes \rho^{\prime}\right)$, our last sentence implies that

$$
\begin{aligned}
\rho\left(\alpha\left(u_{1}\right), \ldots, \alpha\left(u_{m}\right), w_{1}^{\prime \prime}, \ldots, w_{m}^{\prime \prime}, w_{1}, \ldots, w_{2 n}\right) & =\rho_{1}\left(u_{1}, \ldots, u_{m}\right) \rho^{\prime}\left(\delta\left(w_{1}^{\prime \prime}\right), \ldots, \delta\left(w_{m}^{\prime \prime}\right), \delta\left(w_{1}\right), \ldots, \delta\left(w_{2 n}\right)\right) \\
& =\rho_{1}\left(u_{1}, \ldots, u_{m}\right) \rho^{\prime}\left(-u_{1}, \ldots,-u_{m}, \delta\left(w_{1}\right), \ldots, \delta\left(w_{2 n}\right)\right) \\
& =\rho_{1}\left(u_{1}, \ldots, u_{m}\right) \rho^{\prime}\left(u_{1}, \ldots, u_{m}, \delta\left(w_{1}\right), \ldots, \delta\left(w_{2 n}\right)\right) \\
& =\rho_{1}\left(u_{1}, \ldots, u_{m}\right) \rho_{2}\left(u_{1}, \ldots, u_{m}\right) \rho_{3}\left(\vartheta\left(w_{1}\right), \ldots, \vartheta\left(w_{2 n}\right)\right) .
\end{aligned}
$$

We next describe $\nu:=\left(\left(\psi^{\prime}\right)^{-1} \circ\left(\varphi^{\prime}\right)^{-1}\right)\left(\rho_{1} \otimes \rho_{2} \otimes \rho_{3}\right)$. Note that

$$
\left(\varphi^{\prime}\right)^{-1}\left(\rho_{1} \otimes \rho_{2} \otimes \rho_{3}\right)=\nu^{\prime} \otimes \rho_{3},
$$

where $\nu^{\prime} \in\left|T_{m}\left(\Lambda_{1} \cap \Lambda_{2}\right) \oplus T_{m}\left(\Lambda_{1} \cap \Lambda_{2}\right)\right|^{\frac{1}{2}}$ and

$$
\nu\left(w_{1}^{\prime}, \ldots, w_{m}^{\prime}, w_{1}^{\prime \prime}, \ldots, w_{m}^{\prime \prime}\right)=\rho_{1}\left(u_{1}, \ldots, u_{m}\right) \rho_{2}\left(u_{1}, \ldots, u_{m}\right) .
$$

We also observe that $W$ is a complement of $T_{m}\left(\Lambda_{1} \cap \Lambda_{2}\right) \oplus T_{m}\left(\Lambda_{1} \cap \Lambda_{2}\right)$ in $T_{m} \Lambda_{1} \oplus T_{m} \Lambda_{2}$. Since $\nu=\left(\psi^{\prime}\right)^{-1}\left(\nu^{\prime} \otimes \rho_{3}\right)$, the previous sentence implies that

$$
\begin{aligned}
\nu\left(w_{1}^{\prime}, \ldots, w_{m}^{\prime}, w_{1}^{\prime \prime}, \ldots, w_{m}^{\prime \prime}, w_{1}, \ldots, w_{2 n}\right) & =\nu^{\prime}\left(w_{1}^{\prime}, \ldots, w_{m}^{\prime}, w_{1}^{\prime \prime}, \ldots, w_{m}^{\prime \prime}\right) \rho_{3}\left(\vartheta\left(w_{1}\right), \ldots, \vartheta\left(w_{2 n}\right)\right) \\
& =\rho_{1}\left(u_{1}, \ldots, u_{m}\right) \rho_{2}\left(u_{1}, \ldots, u_{m}\right) \rho_{3}\left(\vartheta\left(w_{1}\right), \ldots, \vartheta\left(w_{2 n}\right)\right) .
\end{aligned}
$$

Now consider the linear automorphism $A: T_{m} \Lambda_{1} \oplus T_{m} \Lambda_{2} \longrightarrow T_{m} \Lambda_{1} \oplus T_{m} \Lambda_{2}$ that sends the ordered basis $\left\{w_{1}^{\prime}, \ldots, w_{m}^{\prime}, w_{1}^{\prime \prime}, \ldots, w_{m}^{\prime \prime}, w_{1}, \ldots, w_{2 n}\right\}$ to the ordered basis $\left\{\alpha\left(u_{1}\right), \ldots, \alpha\left(u_{m}\right), w_{1}^{\prime \prime}, \ldots, w_{m}^{\prime \prime}, w_{1}, \ldots, w_{2 n}\right\}$. This automorphism satisfies $\operatorname{det}(A)=1$, so that

$$
\nu\left(w_{1}^{\prime}, \ldots, w_{m}^{\prime}, w_{1}^{\prime \prime}, \ldots, w_{m}^{\prime \prime}, w_{1}, \ldots, w_{2 n}\right)=\nu\left(\alpha\left(u_{1}\right), \ldots, \alpha\left(u_{m}\right), w_{1}^{\prime \prime}, \ldots, w_{m}^{\prime \prime}, w_{1}, \ldots, w_{2 n}\right) .
$$

Along with (60) and (61), this implies that $\rho$ and $\nu$ have the same value on a basis of $T_{m} \Lambda_{1} \oplus T_{m} \Lambda_{2}$. It follows that $\rho=\nu$, completing the proof. 
A5. A reformulation of the BKS density. We again take $M$ to be a manifold equipped with a two-form $\omega \in \Omega^{2}(M)$ and cleanly intersecting Lagrangian submanifolds $\Lambda_{1}, \Lambda_{2} \subseteq M$. Fix a point $m \in \Lambda_{1} \cap \Lambda_{2}$ and let $V_{1}$ and $V_{2}$ be complements of $T_{m}\left(\Lambda_{1} \cap \Lambda_{2}\right)$ in $T_{m} \Lambda_{1}$ and $T_{m} \Lambda_{2}$, respectively, i.e.

$$
T_{m} \Lambda_{1}=T_{m}\left(\Lambda_{1} \cap \Lambda_{2}\right) \oplus V_{1} \quad \text { and } \quad T_{m} \Lambda_{2}=T_{m}\left(\Lambda_{1} \cap \Lambda_{2}\right) \oplus V_{2} .
$$

Assume that $V_{1} \cap V_{2}=\{0\}$ and recall the map

$$
\delta: T_{m} \Lambda_{1} \oplus T_{m} \Lambda_{2} \longrightarrow T_{m} \Lambda_{1}+T_{m} \Lambda_{2}
$$

from Section A3,

Lemma A5.1. The map

$$
\left.\delta\right|_{V_{1} \oplus V_{2}}: V_{1} \oplus V_{2} \longrightarrow T_{m} \Lambda_{1}+T_{m} \Lambda_{2}
$$

is injective, and $\delta\left(V_{1} \oplus V_{2}\right)$ is a complement of $T_{m}\left(\Lambda_{1} \cap \Lambda_{2}\right)$ in $T_{m} \Lambda_{1}+T_{m} \Lambda_{2}$.

Proof. Since $V_{1} \cap V_{2}=\{0\}, V_{1} \oplus V_{2}$ has a trivial intersection with $\operatorname{ker}(\delta)$. It follows that $\left.\delta\right|_{V_{1} \oplus V_{2}}$ is injective and

$$
\begin{aligned}
\operatorname{dim}\left(\delta\left(V_{1} \oplus V_{2}\right)\right) & =\operatorname{dim}\left(V_{1}\right)+\operatorname{dim}\left(V_{2}\right) \\
& =\left(\operatorname{dim}\left(T_{m} \Lambda_{1}\right)-\operatorname{dim}\left(T_{m}\left(\Lambda_{1} \cap \Lambda_{2}\right)\right)\right)+\left(\operatorname{dim}\left(T_{m} \Lambda_{2}\right)-\operatorname{dim}\left(T_{m}\left(\Lambda_{1} \cap \Lambda_{2}\right)\right)\right) \\
& =\operatorname{dim}\left(T_{m} \Lambda_{1}+T_{m} \Lambda_{2}\right)-\operatorname{dim}\left(T_{m}\left(\Lambda_{1} \cap \Lambda_{2}\right)\right) .
\end{aligned}
$$

We are therefore reduced to proving that $T_{m}\left(\Lambda_{1} \cap \Lambda_{2}\right)+\delta\left(V_{1} \oplus V_{2}\right)=T_{m} \Lambda_{1}+T_{m} \Lambda_{2}$. At the same time, we observe that $\delta\left(V_{1} \oplus V_{2}\right)=V_{1}+V_{2}$. This yields

$$
\begin{aligned}
T_{m}\left(\Lambda_{1} \cap \Lambda_{2}\right)+\delta\left(V_{1} \oplus V_{2}\right) & =T_{m}\left(\Lambda_{1} \cap \Lambda_{2}\right)+V_{1}+V_{2} \\
& =\left(T_{m}\left(\Lambda_{1} \cap \Lambda_{2}\right)+V_{1}\right)+\left(T_{m}\left(\Lambda_{1} \cap \Lambda_{2}\right)+V_{2}\right) \\
& =T_{m} \Lambda_{1}+T_{m} \Lambda_{2},
\end{aligned}
$$

completing the proof.

Now recall the map

$$
\beta: T_{m} \Lambda_{1}+T_{m} \Lambda_{2} \longrightarrow \frac{T_{m} \Lambda_{1}+T_{m} \Lambda_{2}}{T_{m}\left(\Lambda_{1} \cap \Lambda_{2}\right)}
$$

from Section A3

Corollary A5.2. The map

$$
\left.(\beta \circ \delta)\right|_{V_{1} \oplus V_{2}}: V_{1} \oplus V_{2} \longrightarrow \frac{T_{m} \Lambda_{1}+T_{m} \Lambda_{2}}{T_{m}\left(\Lambda_{1} \cap \Lambda_{2}\right)}
$$

is an isomorphism.

We have canonical isomorphisms

$$
\begin{aligned}
T_{m} \Lambda_{1} \oplus T_{m} \Lambda_{2} & \cong\left(T_{m}\left(\Lambda_{1} \cap \Lambda_{2}\right) \oplus V_{1}\right) \oplus\left(T_{m}\left(\Lambda_{1} \cap \Lambda_{2}\right) \oplus V_{2}\right) \\
& \cong\left(T_{m}\left(\Lambda_{1} \cap \Lambda_{2}\right) \oplus T_{m}\left(\Lambda_{1} \cap \Lambda_{2}\right)\right) \oplus\left(V_{1} \oplus V_{2}\right)
\end{aligned}
$$


It follows that

$\left|T_{m} \Lambda_{1}\right|^{\frac{1}{2}} \otimes\left|T_{m} \Lambda_{2}\right|^{\frac{1}{2}} \cong\left(\left|T_{m}\left(\Lambda_{1} \cap \Lambda_{2}\right)\right|^{\frac{1}{2}} \otimes\left|V_{1}\right|^{\frac{1}{2}}\right) \otimes\left(\left|T_{m}\left(\Lambda_{1} \cap \Lambda_{2}\right)\right|^{\frac{1}{2}} \otimes\left|V_{2}\right|^{\frac{1}{2}}\right) \quad$ [by (48) and (62)]

$$
\begin{aligned}
& \cong\left|T_{m}\left(\Lambda_{1} \cap \Lambda_{2}\right) \oplus T_{m}\left(\Lambda_{1} \cap \Lambda_{2}\right)\right|^{\frac{1}{2}} \otimes\left|V_{1} \oplus V_{2}\right|^{\frac{1}{2}} \\
& \cong\left|T_{m}\left(\Lambda_{1} \cap \Lambda_{2}\right) \oplus T_{m}\left(\Lambda_{1} \cap \Lambda_{2}\right)\right|^{\frac{1}{2}} \otimes\left|\frac{T_{m} \Lambda_{1}+T_{m} \Lambda_{2}}{T_{m}\left(\Lambda_{1} \cap \Lambda_{2}\right)}\right|^{\frac{1}{2}} \\
& \cong\left|T_{m}\left(\Lambda_{1} \cap \Lambda_{2}\right)\right|
\end{aligned}
$$

\section{[by Corollary $\AA 5.2$}

[by (47), (48), and (52)].

Composing the isomorphisms (66) results in an isomorphism

$$
\Phi_{m}^{V_{1}, V_{2}}:\left|T_{m} \Lambda_{1}\right|^{\frac{1}{2}} \otimes\left|T_{m} \Lambda_{2}\right|^{\frac{1}{2}} \stackrel{\cong}{\longrightarrow}\left|T_{m}\left(\Lambda_{1} \cap \Lambda_{2}\right)\right| .
$$

Proposition A5.3. We have $\Phi_{m}^{V_{1}, V_{2}}=\Phi_{m}$.

Proof. Let $\gamma_{1}: T_{m} \Lambda_{1} \longrightarrow V_{1}$ and $\gamma_{2}: T_{m} \Lambda_{2} \longrightarrow V_{2}$ denote the projections induced by the decompositions $T_{m} \Lambda_{1}=T_{m}\left(\Lambda_{1} \cap \Lambda_{2}\right) \oplus V_{1}$ and $T_{m} \Lambda_{2}=T_{m}\left(\Lambda_{1} \cap \Lambda_{2}\right) \oplus V_{2}$, respectively. We then have a commutative diagram

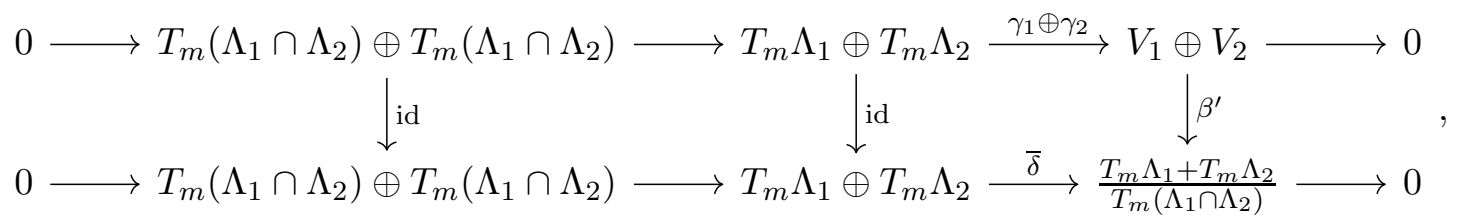

with exact rows, where $\beta^{\prime}$ is the isomorphism obtained by restricting $\beta$ to $V_{1} \oplus V_{2} \subseteq T_{m} \Lambda_{1} \oplus T_{m} \Lambda_{2}$ and $\bar{\delta}$ is the map to $\frac{T_{m} \Lambda_{1}+T_{m} \Lambda_{2}}{T_{m}\left(\Lambda_{1} \cap \Lambda_{2}\right)}$ induced by $\delta$. This induces a commutative diagram

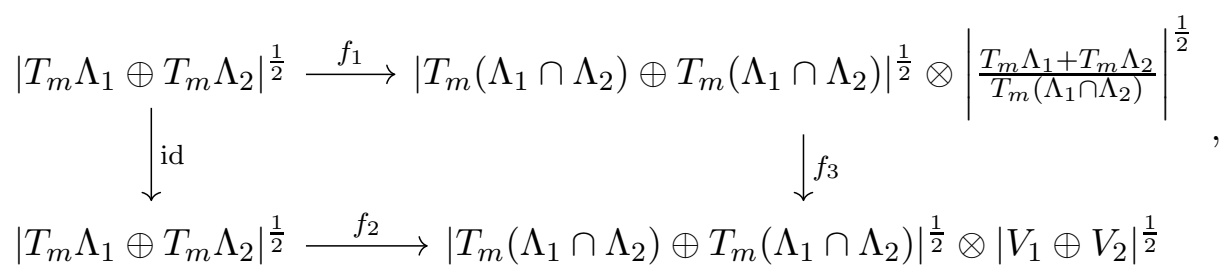

where $f_{3}=\mathrm{id} \otimes\left(\beta^{\prime}\right)^{*}$ and the upper (resp. lower) horizontal map is induced by the lower (resp. upper) row in the previous diagram. On the other hand, applying Lemma A5.1 to the exact sequences

$$
0 \longrightarrow T_{m}\left(\Lambda_{1} \cap \Lambda_{2}\right) \longrightarrow T_{m} \Lambda_{1} \stackrel{\gamma_{1}}{\longrightarrow} V_{1} \longrightarrow 0 \quad \text { and } \quad 0 \longrightarrow T_{m}\left(\Lambda_{1} \cap \Lambda_{2}\right) \longrightarrow T_{m} \Lambda_{2} \stackrel{\gamma_{2}}{\longrightarrow} V_{2} \longrightarrow 0
$$

gives rise to a commutative diagram

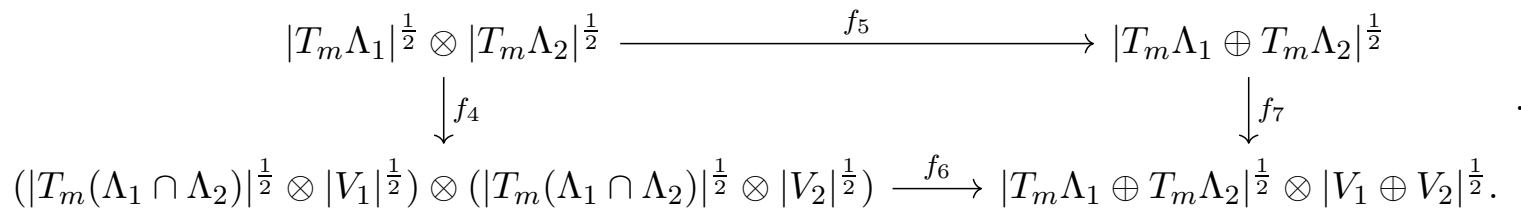


We also have a commutative diagram

$$
\begin{aligned}
\left|T_{m}\left(\Lambda_{1} \cap \Lambda_{2}\right) \oplus T_{m}\left(\Lambda_{1} \cap \Lambda_{2}\right)\right|^{\frac{1}{2}} \otimes\left|\frac{T_{m} \Lambda_{1}+T_{m} \Lambda_{2}}{T_{m}\left(\Lambda_{1} \cap \Lambda_{2}\right)}\right|^{\frac{1}{2}} & \downarrow f_{8} \\
& \downarrow
\end{aligned}
$$

where $f_{8}, f_{9}$, and $f_{10}$ are the isomorphisms defined in (58b), (66d), and (55d), respectively. Lemma A4.1 then combines with the definition of $\Phi_{m}$ to imply that $\Phi_{m}=f_{10} \circ f_{8} \circ f_{1} \circ f_{5}$, while the definition of $\Phi_{m}^{V_{1}, V_{2}}$ gives $\Phi_{m}^{V_{1}, V_{2}}=f_{9} \circ f_{3}^{-1} \circ f_{6} \circ f_{4}$. The equality $\Phi_{m}^{V_{1}, V_{2}}=\Phi_{m}$ now follows easily from the fact that (68), (69), and (70) commute.

A6. A result on half-densities and $G$-invariance. Recall the Lie-theoretic notation and conventions established in Section [3.1, as well as the BKS density $D\left(\rho_{1}, \rho_{2}\right)$ from Section A3. This density is compatible with group actions in the following sense.

Lemma A6.1. Let $M$ be a manifold equipped with a smooth $G$-action and a $G$-invariant two-form $\omega \in \Omega^{2}(M)^{G}$. Suppose that $\Lambda_{1}, \Lambda_{2} \subseteq M$ are $G$-invariant Lagrangian submanifolds having a clean intersection. If $\rho_{1}$ and $\rho_{2}$ are $G$-invariant half-densities on $\Lambda_{1}$ and $\Lambda_{2}$, respectively, then $D\left(\rho_{1}, \rho_{2}\right)$ is a $G$-invariant half-density on $\Lambda_{1} \cap \Lambda_{2}$.

Proof. Fix $g \in G$ and $m \in M$. Observe that $g$ acts on $M$ through a diffeomorphism $M \longrightarrow M$ whose differential defines isomorphisms

$$
\begin{aligned}
& T_{m} \Lambda_{1} \stackrel{\cong}{\longrightarrow} T_{g \cdot m} \Lambda_{1}, \\
& T_{m} \Lambda_{2} \cong T_{g \cdot m} \Lambda_{2}, \\
& T_{m}\left(\Lambda_{1} \cap \Lambda_{2}\right) \stackrel{\cong}{\longrightarrow} T_{g \cdot m}\left(\Lambda_{1} \cap \Lambda_{2}\right) \\
& T_{m} \Lambda_{1}+T_{m} \Lambda_{2} \cong T_{g \cdot m} \Lambda_{1}+T_{g \cdot m} \Lambda_{2}, \\
& \frac{T_{m} \Lambda_{1}+T_{m} \Lambda_{2}}{T_{m}\left(\Lambda_{1} \cap \Lambda_{2}\right)} \stackrel{\cong}{\frac{T_{g \cdot m} \Lambda_{1}+T_{g \cdot m} \Lambda_{2}}{T_{g \cdot m}\left(\Lambda_{1} \cap \Lambda_{2}\right)} .} .
\end{aligned}
$$

Note that (71a), (71b), and their direct sum fit into the commutative diagram

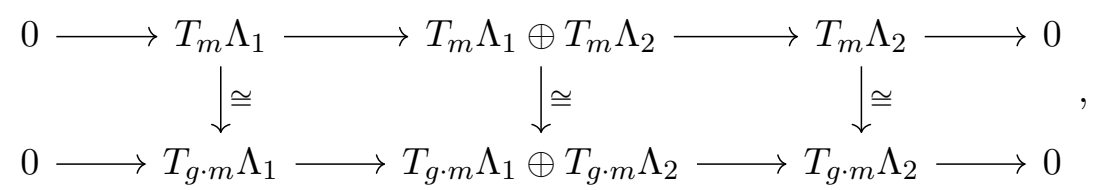

where $T_{m} \Lambda_{1} \longrightarrow T_{m} \Lambda_{1} \oplus T_{m} \Lambda_{2}$ and $T_{g \cdot m} \Lambda_{1} \longrightarrow T_{g \cdot m} \Lambda_{1} \oplus T_{g \cdot m} \Lambda_{2}$ are the usual inclusions and $T_{m} \Lambda_{1} \oplus$ $T_{m} \Lambda_{2} \longrightarrow T_{m} \Lambda_{2}$ and $T_{g \cdot m} \Lambda_{1} \oplus T_{g \cdot m} \Lambda_{2} \longrightarrow T_{g \cdot m} \Lambda_{2}$ are the usual projections. The isomorphisms (171) also connect (53) and (54) at the point $m$ to (53) and (54) at the point $g \cdot m$, respectively. A 
more precise statement is that we have the commutative diagrams

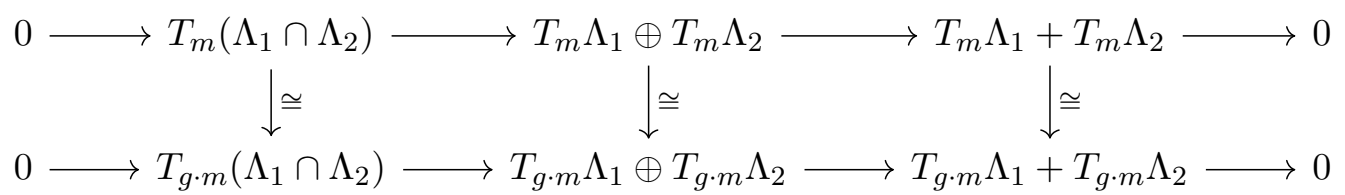

and

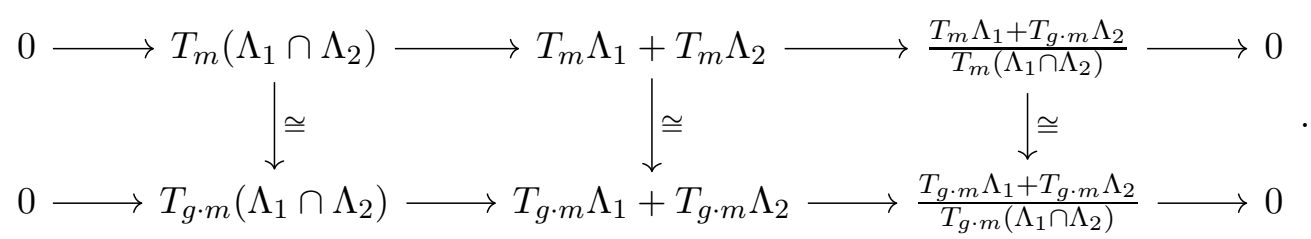

By applying Lemma A2.2 to (72), (73), and (74), we obtain the commutative diagrams

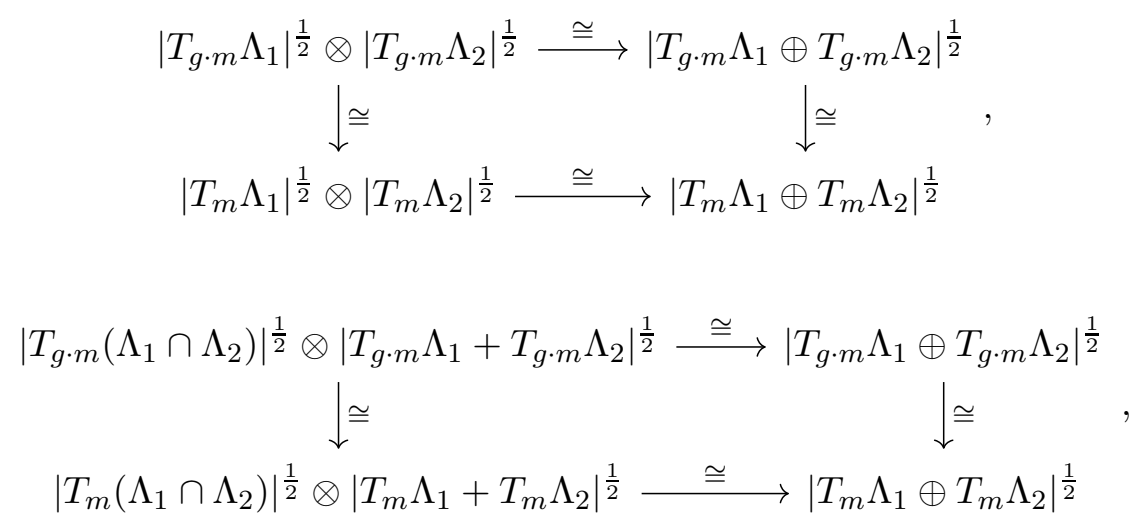

and

$$
\begin{aligned}
& \left|T_{g \cdot m}\left(\Lambda_{1} \cap \Lambda_{2}\right)\right|^{\frac{1}{2}} \otimes\left|\frac{T_{g \cdot m} \Lambda_{1}+T_{g \cdot m} \Lambda_{2}}{T_{g \cdot m}\left(\Lambda_{1} \cap \Lambda_{2}\right)}\right|^{\frac{1}{2}} \cong\left|T_{g \cdot m} \Lambda_{1}+T_{g \cdot m} \Lambda_{2}\right|^{\frac{1}{2}} \\
& \downarrow \cong \\
& \left|T_{m}\left(\Lambda_{1} \cap \Lambda_{2}\right)\right|^{\frac{1}{2}} \otimes\left|\frac{T_{m} \Lambda_{1}+T_{m} \Lambda_{2}}{T_{m}\left(\Lambda_{1} \cap \Lambda_{2}\right)}\right|^{\frac{1}{2}} \cong\left|T_{m} \Lambda_{1}+T_{m} \Lambda_{2}\right|^{\frac{1}{2}}
\end{aligned}
$$

Let us again consider the diffeomorphism $M \longrightarrow M$ through which $g$ acts on $M$. Since this diffeomorphism preserves $\omega,(\overline{71 e})$ is an isomorphism of symplectic vector spaces. It follows that

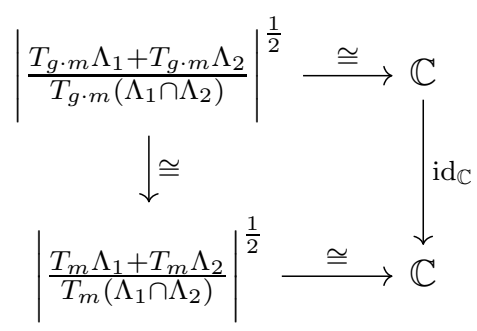


commutes, where the leftmost vertical map is induced by (71e) and the horizontal maps are (52) at the points $m$ and $g \cdot m$. We also observe that

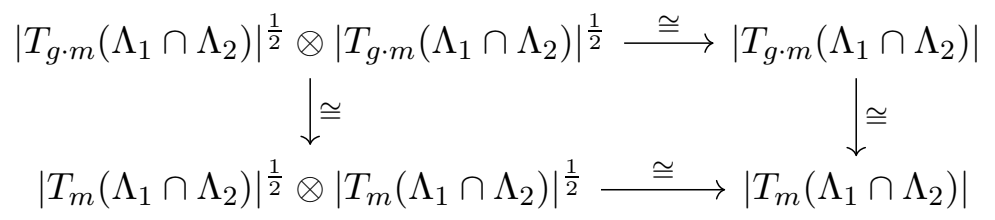

commutes, where the vertical maps are induced by (171c) and the horizontal maps are specific instances of (47).

Now recall that (56) is obtained by composing the isomorphisms (55). With this point in mind, one can combine (75), (76), (77), (78), and (79) to produce the following commutative diagram:

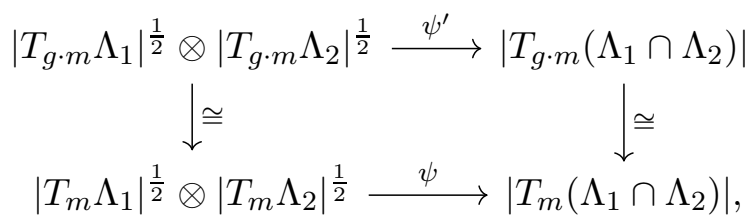

where horizontal maps are (56) at $m$ and $g \cdot m$, the leftmost vertical map comes from (71a) and (71b), and the rightmost vertical map is induced by (71c). Since $\rho_{1}$ and $\rho_{2}$ are $G$-invariant, $\left(\rho_{1}\right)_{m} \otimes\left(\rho_{2}\right)_{m}$ is the image of $\left(\rho_{1}\right)_{g \cdot m} \otimes\left(\rho_{2}\right)_{g \cdot m}$ under the leftmost vertical map. It follows that $\psi\left(\left(\rho_{1}\right)_{m} \otimes\left(\rho_{2}\right)_{m}\right)$ is the image of $\psi^{\prime}\left(\left(\rho_{1}\right)_{g \cdot m} \otimes\left(\rho_{2}\right)_{g \cdot m}\right)$ under the rightmost vertical map. One also has $\psi\left(\left(\rho_{1}\right)_{m} \otimes\left(\rho_{2}\right)_{m}\right)=$ $D\left(\rho_{1}, \rho_{2}\right)_{m}$ and $\psi^{\prime}\left(\left(\rho_{1}\right)_{g \cdot m} \otimes\left(\rho_{2}\right)_{g \cdot m}\right)=D\left(\rho_{1}, \rho_{2}\right)_{g \cdot m}$, which combine with the previous sentence to show that $D\left(\rho_{1}, \rho_{2}\right)$ is $G$-invariant.

\section{NOTATION}

- $Q(M)$ - quantization of $M$

- $M_{1} \stackrel{\Lambda}{\Longrightarrow} M_{2}$ - Lagrangian relation

- $M^{\circ}$ - open submanifold of $M$ on which a given two-form is non-degenerate

- $D\left(\rho_{1}, \rho_{2}\right)$ - BKS density determined by the half-densities $\rho_{1}$ and $\rho_{2}$

- $G$ - compact, connected, simply-connected, simple Lie group

- $n$ - dimension of $G$

- $r$ - rank of $G$

- $\theta^{L}$ - left-invariant Maurer-Cartan form on $G$

- $\theta^{R}$ — right-invariant Maurer-Cartan form on $G$

- $G_{\text {reg }}$ - regular elements of $G$

- $\mathcal{C}$ - conjugacy class in $G$

- $\Lambda_{\mathcal{C}}$ - submanifold of points $(g, h) \in D(G)$ satisfying $g h g^{-1}=h$ and $h \in \mathcal{C}$

- $G_{x}-G$-stabilizer of a point $x$ in a $G$-manifold

- $G x-G$-orbit of a point $x$ in a $G$-manifold

- $\mathfrak{g}$ - Lie algebra of $G$

- $\mathfrak{g}_{x}$ - Lie algebra of $G_{x}$

- $\xi_{M}$ - fundamental vector field of $\xi \in \mathfrak{g}$ on a $G$-manifold $M$

- $\Theta_{G_{\xi}}$ - left-invariant form volume form on $G_{\xi}$ that induces the Haar measure, where $\xi \in \mathfrak{g}$

- $\Theta_{\xi}$ - value of $\Theta_{G_{\xi}}$ at $e \in G_{\xi}$, where $\xi \in \mathfrak{g}$

- $\langle\cdot, \cdot\rangle$ - normalized inner product on $\mathfrak{g}$

- $V^{\perp}$ — orthogonal complement of a subspace $V \subseteq \mathfrak{g}$ with respect to $\langle\cdot, \cdot\rangle$

- $\mathfrak{g}_{\text {reg }}$ - regular elements of $\mathfrak{g}$ 
- $\exp$ - exponential map $\mathfrak{g} \longrightarrow G$

- $\operatorname{Ad}_{g}(\xi)$ - adjoint action of $g \in G$ on $\xi \in \mathfrak{g}$

- $\mathcal{O}$ - adjoint orbit of $G$ in $\mathfrak{g}$

- $\Lambda_{\mathcal{O}}$ - submanifold of points $(g, \xi) \in G \times \mathfrak{g}$ satisfying $\operatorname{Ad}_{g}(\xi)=\xi$ and $\xi \in \mathcal{O}$

- $\omega_{\mathcal{O}}$ - KKS symplectic form on $\mathcal{O}$

- $\omega_{\xi}$ - value of $\omega_{\mathcal{O}}$ at $\xi \in \mathcal{O}$

- $\Omega_{\mathcal{O}}$ - highest non-zero wedge power of $\omega_{\mathcal{O}}$

- $\Omega_{\xi}$ - value of $\Omega_{\mathcal{O}}$ at $\xi \in \mathcal{O}$

- $\rho_{\mathcal{O}}$ - Guillemin-Sternberg half-density on $\Lambda_{\mathcal{O}}$

- $\psi_{\mathcal{O}}$ - phase function on $\Lambda_{\mathcal{O}}$

- $\rho_{\mathcal{C}}$ - half-density on $\Lambda_{\mathcal{C}}$

- $\psi_{\mathcal{C}}$ - phase function on $\Lambda_{\mathcal{C}}$

- $R$ - Dehn twist automorphism $D(G) \longrightarrow D(G)$ defined by $R(g, h)=\left(g, h g^{-1}\right)$

- $\nu_{\mathcal{C}}$ - half-density on $R\left(\Lambda_{\mathcal{C}}\right)$ determined by $\mathcal{O}$

- $\vartheta_{\mathcal{C}}$ - phase function on $R\left(\Lambda_{\mathcal{C}}\right)$

- $\omega_{D(G)}$ - two-form on the quasi-Hamiltonian $G$-space $D(G)$

- $\mu_{D(G)}$ - moment map on the quasi-Hamiltonian $G$-space $D(G)$

- $\mu_{D(G)}^{-1}(e)_{\mathrm{reg}}$ - intersection of $\mu_{D(G)}^{-1}(e)$ and $D(G)_{\mathrm{reg}}$ in $D(G)$

- $\overline{\omega_{D(G)}}$ - symplectic form on $\mu_{D(G)}^{-1}(e)_{\mathrm{reg}} / G$

- $T$ - maximal torus in $G$

- $\mathfrak{t}$ - Lie algebra of $T$

- $W$ - Weyl group of $(G, T)$

- $\Phi$ - roots of $(G, T)$

- $\Delta$ - chosen set of simple roots in $\Phi$

- $\ell(w)$ - length of $w \in W$ with respect to $\Delta$

- $T_{\text {reg }}$ - intersection of $T$ and $G_{\text {reg }}$ in $G$

- $\omega_{T \times T}$ - symplectic form on $T \times T$

- $\frac{T \times T}{\omega_{T \times T}}$ - symplectic form on $\left(T \times T_{\mathrm{reg}}\right) / W$

\section{REFERENCES}

[1] Alekseev, A., Malkin, A., And Meinrenken, E. Lie group valued moment maps. J. Differential Geom. 48, 3 (1998), 445-495.

[2] Alekseev, A., Meinrenken, E., And Woodward, C. The Verlinde formulas as fixed point formulas. J. Symplectic Geom. 1, 1 (2001), 1-46.

[3] Bates, S., And Weinstein, A. Lectures on the geometry of quantization, vol. 8 of Berkeley Mathematics Lecture Notes. American Mathematical Society, Providence, RI; Berkeley Center for Pure and Applied Mathematics, Berkeley, CA, 1997.

[4] Blattner, R. J. Quantization and representation theory. In Harmonic analysis on homogeneous spaces (Proc. Sympos. Pure Math., Vol. XXVI, Williams Coll., Williamstown, Mass., 1972) (1973), pp. 147-165.

[5] Goldman, W. M. The modular group action on real SL(2)-characters of a one-holed torus. Geom. Topol. 7 (2003), 443-486.

[6] Guillemin, V., And Sternberg, S. Semi-classical analysis. International Press, Boston, MA, 2013.

[7] Laurent-Gengoux, C., And Xu, P. Quantization of pre-quasi-symplectic groupoids and their Hamiltonian spaces. In The breadth of symplectic and Poisson geometry, vol. 232 of Progr. Math. Birkhäuser Boston, Boston, MA, 2005, pp. 423-454.

[8] Loizides, Y., And Song, Y. Quantization of Hamiltonian loop group spaces. Math. Ann. 374, 1-2 (2019), 681-722.

[9] Loizides, Y., And Song, Y. Norm-square localization and the quantization of Hamiltonian loop group spaces. J. Funct. Anal. 278, 9 (2020), 108445. 
TOWARDS A QUANTIZATION OF THE DOUBLE VIA THE ENHANCED SYMPLECTIC "CATEGORY"

[10] Meinrenken, E. Quantization of $q$-Hamiltonian SU(2)-spaces. In Geometric aspects of analysis and mechanics, vol. 292 of Progr. Math. Birkhäuser/Springer, New York, 2011, pp. 257-293.

[11] Meinrenken, E. Lectures on group-valued moment maps and Verlinde formulas. In Mathematical aspects of quantization, vol. 583 of Contemp. Math. Amer. Math. Soc., Providence, RI, 2012, pp. 175-210.

[12] Meinrenken, E. Twisted K-homology and group-valued moment maps. Int. Math. Res. Not. IMRN, 20 (2012), 4563-4618.

[13] Meinrenken, E., And Woodward, C. Moduli spaces of flat connections on 2-manifolds, cobordism, and Witten's volume formulas. In Advances in geometry, vol. 172 of Progr. Math. Birkhäuser Boston, Boston, MA, 1999, pp. 271-295.

[14] Shahbazi, Z. Prequantization of quasi-Hamiltonian spaces. Int. Math. Res. Not. (2006), Art. ID $29354,22$.

[15] Song, Y. Dirac operators on quasi-Hamiltonian G-spaces. J. Geom. Phys. 106 (2016), 70-86.

[16] Weinstein, A. Symplectic categories. Port. Math. 67, 2 (2010), 261-278.

Department of Mathematics, Northeastern University, 360 Huntington Avenue, Boston, MA 02115, USA

Email address, Peter Crooks: p.crooks@northeastern.edu

Email address, Jonathan Weitsman: j.weitsman@northeastern.edu 